\title{
Um estudo de simulação para comparação entre métodos de cálculo do número aproximado de graus de liberdade da estatística $F$ em dados desbalanceados
}

Andréia Pereira Maria Hilário

Dissertação apresentada para obtenção do título de Mestra em Ciências. Área de concentração: Estatística e Experimentação Agronômica 
Andréia Pereira Maria Hilário

Licenciada em Matemática

Um estudo de simulação para comparação entre métodos de cálculo do número aproximado de graus de liberdade da estatística $F$ em dados desbalanceados

versão revisada de acordo com a resolução CoPGr 6018 de 2011

Orientador:

Prof. Dr. CÉSAR GONÇALVES DE LIMA

Dissertação apresentada para obtenção do título de Mestra em Ciências. Área de concentração: Estatística e Experimentação Agronômica 


\section{Dados Internacionais de Catalogação na Publicação DIVISÃO DE BIBLIOTECA - DIBD/ESALQ/USP}

Hilário, Andréia Pereira Maria

Um estudo de simulação para comparação entre métodos de cálculo do número aproximado de graus de liberdade da estatística $F$ em dados desbalanceados / Andréia Pereira Maria Hilário. - - versão revisada de acordo com a resolução CoPGr 6018 de 2011. - - Piracicaba, 2015.

81 p. : il.

Dissertação (Mestrado) - - Escola Superior de Agricultura "Luiz de Queiroz".

1. Poder de teste 2. Taxa de erro tipo I 3. Graus de liberdade 4. Método de Kenward-Roger 5. Método de Satterthwaite I. Título

CDD 519.5

$\mathrm{H} 641 \mathrm{e}$

"Permitida a cópia total ou parcial deste documento, desde que citada a fonte - $\mathrm{O}$ autor" 


\section{DEDICATÓRIA}

Dedico este trabalho aos meus pais, Clarice e Benedito, que me apoiaram com ensinamentos preciosos.

Aos meus filhos, Giselda e José, que são os amores de minha vida.

Ao meu querido esposo, Reginaldo, pelo amor e paciência, por sua compreensão e companheirismo. 


\section{AGRADECIMENTOS}

Agradeço primeiramente a Deus, pelo dom da vida, pelo amparo e infinito amor, por sua divina graça sempre presente em minha vida.

Aos meus pais, Clarice e Benedito, ao meu irmão, André, que me apoiaram com muito carinho e dedicação.

Ao Prof. Dr. César Gonçalves de Lima, pela confiança em mim depositada, pela orientação, pela amizade, pela compreensão e dedicação.

A Profa. Dra. Clarice Garcia Borges Demétrio, pelo incentivo e sabedoria compartilhada.

A Profa. Dra. Renata Alcarde Sermarini, pela atenção, pela amizade e incentivo.

Aos professores do Departamento de Ciências Exatas, que foram tão importantes na minha vida acadêmica e no desenvolvimento deste trabalho.

A todos os professores que colaboraram para minha formação, em particular ao Prof. Dr. Silvano Cesar da Costa e a Profa. Dra. Elizabeth Strapasson da Universidade Estadual de Londrina, que me incentivaram e me apoiaram a continuar os estudos.

A minha família, pela orientação, dedicação, incentivo, paciência nessa fase de minha vida, em especial ao meu esposo e aos meus pequeninos.

Aos colegas do departamento, em especial aos amigos: Maria Cristina Martins, Ricardo K. Sercundes, Alessandra Santos, Erasnilson Vieira Camilo, Valiana Alves Teodoro, Djair D. R. Frade e Thiago de P. Oliveira.

A Cnpq pela concessão da bolsa de estudos.

E a todos que, de forma direta ou indireta, fizeram parte da minha formação. 
"A sabedoria educa os seus filhos e cuida daqueles que a procuram.

Quem tem amor a ela, ama a vida, e os que madrugam para procurá-la, ficarão cheios de alegria.

Quem a possui, terá como herança a honra, e aonde quer que vá, o Senhor o abençoará.

Aqueles que servem a ela prestam culto ao Santo, e o Senhor ama aqueles que a amam.

Quem a escuta, julga com justiça, e quem lhe dá atenção viverá tranquilo. Quem nela confia, a receberá como herança, e seus descendentes a conservarão como propriedade.

Primeiro, ela o conduzirá por caminhos tortuosos, causando-lhe medo e tremor, e o atormentará com sua disciplina, até que o homem confie nela e até que ela o tenha provado com suas exigências.

Depois, ela o reconduzirá pelo caminho reto, o alegrará e lhe manifestará os seus segredos.

Se ele se desviar, ela o abandonará e o entregará ao sabor do próprio destino." 


\section{SUMÁRIO}

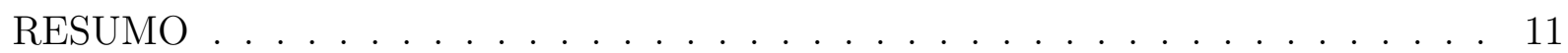

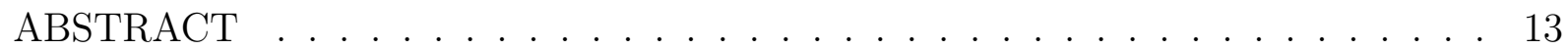

LISTA DE TABELAS . . . . . . . . . . . . . . . . . . 15

1 INTRODUÇÃOO . . . . . . . . . . . . . . . . . . . . . . . . . 17

2 REVISÃO BIBLIOGRÁFICA . . . . . . . . . . . . . . . . . . . . 19

2.1 Teste de Hipóteses . . . . . . . . . . . . . . . . . . . . . . . . . . . . . . . 19

2.1 .1 Erros tipo I e tipo II . . . . . . . . . . . . . . . . . . . . . 20

2.1 .2 Poder de um teste de hipótese . . . . . . . . . . . . . . . . . . . . 20

2.2 Modelos lineares mistos . . . . . . . . . . . . . . . . . . . . . . . 22

2.2 .1 Estruturas da matriz de variâncias e covariâncias . . . . . . . . . . . . . 23

2.2 .2 Estimação dos componentes de variância . . . . . . . . . . . . . . . . . . . 24

2.2.2.1 Método da Máxima Verossimilhança _ . . . . . . . . . . . . . . 25

2.2.2.2 Método da Máxima Verossimilhança Restrita . . . . . . . . . . . . . . 25

2.3 Testes para os efeitos fixos . . . . . . . . . . . . . . . . 26

2.3.1 Teste aproximado $t \ldots \ldots \ldots \ldots \ldots \ldots \ldots$

2.3.2 Teste aproximado Wald- $F \ldots \ldots \ldots$. . . . . . . . . . 27

2.4 Métodos para aproximar o número de graus de liberdade . . . . . . . . . . . 27

2.5 Simulações computacionais . . . . . . . . . . . . . . . . . . . . . . . 34

3 MATERIAL E MÉTODOS . . . . . . . . . . . . . . . . . 35

3.1 Modelos experimentais estudados . . . . . . . . . . . . . . . 35

3.1.1 Delineamento casualizado em blocos (DBC) . . . . . . . . . . . . 35

3.1.2 Delineamento casualizado em blocos com tratamentos no esquema de parcelas subdivididas $(\mathrm{DBC} / \mathrm{PSD}) \ldots \ldots \ldots \ldots \ldots$

3.2 Estrutura dos dados simulados . . . . . . . . . . . . . . . . . . 36

4 RESULTADOS E DISCUSSÃO . . . . . . . . . . . . . . . . . . . . . . 41

4.1 Delineamento casualizado em blocos $(\mathrm{DBC}) \ldots \ldots \ldots 4 . \ldots \ldots$

4.2 Delineamento casualizado em blocos com tratamentos no esquema de parcela subdividida $(\mathrm{DBC} / \mathrm{PSD}) \ldots \ldots \ldots \ldots \ldots \ldots \ldots \ldots$

5 CONCLUSÕES . . . . . . . . . . . . . . . . . . . . . 63

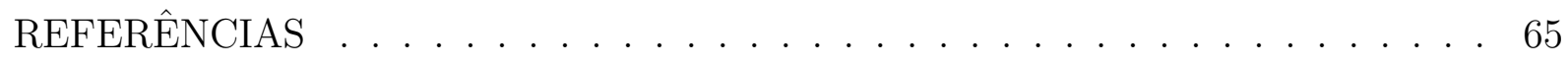

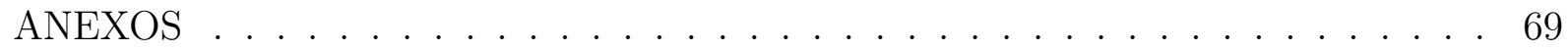




\section{RESUMO}

\section{Um estudo de simulação para comparação entre métodos de cálculo do número aproximado de graus de liberdade da estatística $F$ em dados desbalanceados}

O desbalanceamento de dados em experimentos está muitas vezes presente em diversas pesquisas nas mais variadas áreas do conhecimento. Embora existam muitas maneiras de análise de tais dados, além de diversos recursos computacionais já implementados em diversos softwares estatísticos, ainda perdura dúvidas entre os pesquisadores a respeito da opção de análise mais eficiente. A literatura fornece ao pesquisador direção na escolha da metodologia de análise a obter maior eficácia nos resultados de sua pesquisa, mas o número elevado de opções pode tornar a escolha difícil. Em se tratando de testes estatísticos, algumas das opções para se trabalhar com dados desbalanceados são os testes $t$ e Wald- $F$, mas ainda resta ao pesquisador decidir entre as várias opções disponíveis nos pacotes, pois nem sempre as opções padrões são as mais indicadas. No presente trabalho foram realizadas simulações com diferentes cenários experimentais, utilizando-se o delineamento casualizado em blocos com um fator de tratamento em uma situação e o esquema de tratamentos em parcelas subdividas em outra, sendo comparados quatro métodos de cálculo do número aproximado de graus de liberdade (Containment, Residual, Satterthwaite e Kenward-Roger). Verificou-se que o método de Kenward-Roger controla de maneira mais eficiente a taxa de erro tipo I e não é inferior aos outros métodos com respeito ao poder do teste Wald- $F$.

Palavras-chave: Poder de teste; Taxa de erro tipo I; Graus de liberdade; Método de Kenward-Roger; Método de Satterthwaite 


\begin{abstract}
A simulation study to compare the approximate number calculation methods of degrees of freedom of the $F$ statistic in unbalanced data

The data imbalance in experiments is often present in several researches in various fields of knowledge. While there are many ways to analyze these data in addition to various computer resources already implemented in many statistical software, doubt still lingers among researchers about the most efficient analysis option. The literature provides the researcher direction in choosing the analysis methodology to get better in your search results, but the large number of options can make the difficult choice. When it comes to statistical tests, some of the options for working with unbalanced data are the tests $t$ and Wald- $F$, but there is still the researcher to decide between the various options available in the packages because the defaults are not always the most suitable. This experiment was carried out simulations with different experimental scenarios, using the randomized block design with one factor in a situation treatment and treatment regimen subdivided parcels in another, and compared four methods of calculating the approximate number of degrees of freedom (Containment, Residual, Satterthwaite and Kenward-Roger). It has been found that the method of Kenward-Roger controls more efficiently the type I error rate and is not inferior to other methods with respect to the power of the test Wald- $F$.
\end{abstract}

Keywords: Power test; Type I error rate; Degrees of freedom; Method Kenward-Roger; Method Satterthwaite 


\section{LISTA DE TABELAS}

Tabela 1 - Possíveis situações no teste de hipótese . . . . . . . . . . . . . . 20

Tabela 2 - Descrição dos modelos utilizados nas simulações . . . . . . . . . . . . 37

Tabela 3 - Poder analítico (P.A.), poder simulado (P.S.) e taxa de erro tipo I ( $\hat{\alpha})$ para o Modelo I com níveis de desbalanceamento de 10\%, 20\% e 30\% . . 42

Tabela 4 - Poder analítico (P.A.), poder simulado (P.S.) e taxa de erro tipo I ( $\hat{\alpha})$ para o Modelo II com níveis de desbalanceamento de 10\%, 20\% e 30\% . 43

Tabela 5 - Poder analítico (P.A.), poder simulado (P.S.) e taxa de erro tipo I ( $\hat{\alpha})$ para o Modelo III com níveis de desbalanceamento de 10\%, 20\% e 30\% .

Tabela 6 - Poder analítico (P.A.), poder simulado (P.S.) e taxa de erro tipo I ( $\hat{\alpha})$ para a interação $A B$ no Modelo IV com desbalanceamentos de 10\%, 20\% e $30 \% \ldots \ldots \ldots \ldots \ldots \ldots$

Tabela 7 - Poder analítico (P.A.), poder simulado (P.S.) e taxa de erro tipo I ( $\hat{\alpha})$ para o fator $A$ no Modelo IV com desbalanceamentos de $10 \%, 20 \%$ e $30 \% 47$

Tabela 8 - Poder analítico (P.A.), poder simulado (P.S.) e taxa de erro tipo I ( $\hat{\alpha})$ para o fator $B$ no Modelo IV com desbalanceamentos de 10\%, 20\% e $30 \% 48$

Tabela 9 - Poder analítico (P.A.), poder simulado (P.S.) e taxa de erro tipo I $(\hat{\alpha})$ para o fator $B$ no Modelo VII, considerando $\sigma_{A}^{2}=\sigma_{A B}^{2}=0,1$, com desbalanceamentos de $10 \%, 20 \%$ e $30 \%$. . . . . . . . . . . . 49

Tabela 10 -Poder analítico (P.A.), poder simulado (P.S.) e taxa de erro tipo I $(\hat{\alpha})$ para a interação $A B$ no Modelo V com desbalanceamentos de 10\%, $20 \%$

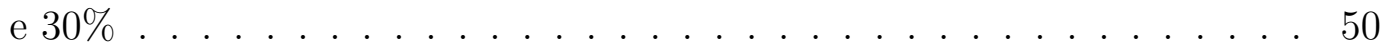

Tabela 11 -Poder analítico (P.A.), poder simulado (P.S.) e taxa de erro tipo I ( $\hat{\alpha})$ para o fator $A$ no Modelo V com desbalanceamentos de $10 \%, 20 \%$ e $30 \% 51$

Tabela 12 -Poder analítico (P.A.), poder simulado (P.S.) e taxa de erro tipo I ( $\hat{\alpha})$ para o fator $B$ no Modelo V com desbalanceamentos de 10\%, 20\% e $30 \% 53$

Tabela 13 -Poder analítico (P.A.), poder simulado (P.S.) e taxa de erro tipo I ( $\hat{\alpha})$ para o fator $B$ no Modelo VIII, considerando $\sigma_{A}^{2}=\sigma_{A B}^{2}=0,1$, com desbalanceamentos de $10 \%, 20 \%$ e $30 \%$. . . . . . . . . 54

Tabela 14 -Poder analítico (P.A.), poder simulado (P.S.) e taxa de erro tipo I $(\hat{\alpha})$ para a interação $A B$ no Modelo VI com desbalanceamentos de 10\%, 20\%

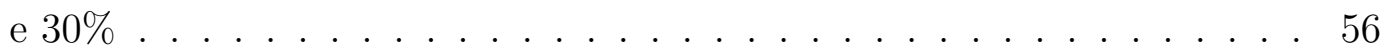


Tabela 15 -Poder analítico (P.A.), poder simulado (P.S.) e taxa de erro tipo I $(\hat{\alpha})$ para o fator $A$ no Modelo VI com desbalanceamentos de 10\%, 20\% e 30\% 57

Tabela 16 -Poder analítico (P.A.), poder simulado (P.S.) e taxa de erro tipo I ( $\hat{\alpha})$ para o fator $B$ no Modelo VI com desbalanceamentos de 10\%, 20\% e $30 \% 59$

Tabela 17 -Poder analítico (P.A.), poder simulado (P.S.) e taxa de erro tipo I $(\hat{\alpha})$ para o fator $B$ no Modelo IX, considerando $\sigma_{A}^{2}=\sigma_{A B}^{2}=0,1$, com desbalanceamentos de $10 \%, 20 \%$ e $30 \%$. . . . . . . . . 60 


\section{INTRODUÇÃO}

O desbalanceamento de dados em experimentos é muitas vezes inevitável e chega a surpreender até os pesquisadores mais precavidos, porém um devido e necessário tratamento na análise de tais dados pode amenizar os impactos negativos nos resultados causados pela perda de unidades experimentais.

Um pesquisador prevenido pode antecipar as perdas de unidades experimentais e utilizar um número maior de unidades, mas muitas vezes é inviável a utilização de unidades excedentes, por questões práticas ou porque os recursos de execução da pesquisa em si não a permitem.

Uma das maneiras de obter maior eficiência na análise de dados desbalanceados é saber quais são os recursos disponíveis na análise de tais dados, mais que isso, é saber qual a escolha do recurso de análise que proporciona resultados mais efetivos. Em se tratando de testes estatísticos, há diversas formas de análise já implementadas em diversos softwares estatísticos, mas na maioria das vezes as opções padrões dos softwares não são o modo mais eficaz de se analisar os dados. As inúmeras opções existentes tornam a escolha ainda mais difícil e uma escolha equivocada pode comprometer os resultados das análises ou gerar perda de eficiência das mesmas.

$\mathrm{Na}$ literatura pode-se consultar muitas descrições de resultados de análises que, de forma geral, direcionam os pesquisadores a escolherem um modo de análise a obter maior eficácia nos resultados de suas pesquisas. Quando há desbalanceamento dos dados, atualmente é frequente a utilização de modelos lineares mistos para acomodar tal situação e realizar a análise de maneira conveniente (SPILKE et al. 2005).

Muitas situações levam delineamentos balanceados a se tornarem desbalanceados por circunstâncias, geralmente, imprevistas e há casos em que delineamentos podem ser considerados naturalmente desbalanceados. Neste último caso, pode-se citar por exemplo, os experimentos em que se estuda a aptidão de produção de leite em vacas leiteiras, quando não é garantido que os touros envolvidos na pesquisa tenham o mesmo número de descendentes que o número de vacas.

Enquanto que para dados balanceados, as estimativas de componentes de variância baseadas no método dos momentos (método ANOVA) apresentam quadrados médios com propriedades ótimas (PIEPHO; SPILKE, 1999), para dados desbalanceados isso não ocorre. Na presença de dados desbalanceados Searle et al. (1992) recomen- 
dam a utilização do método da máxima verossimilhança restrita (REML), que teve sua eficácia estudada por meio de simulações com diferentes estruturas de dados (SPILKE; TUCHSCHERER, 2001; GUIARD; SPILKE; DÄNICKE, 2003).

Os testes estatísticos para um modelo linear com dados balanceados, assumindo os pressupostos de normalidade, homogeneidade de variâncias e independência dos efeitos aleatórios do modelo, já estão bem estabelecidos e são frequentemente resumidos por um quadro de análise de variância. Nesse caso, em que os dados são balanceados, cada soma de quadrados é independente uma da outra e segue uma distribuição $\chi^{2}$. Para a maioria dessas situações, estas somas de quadrados podem ser utilizadas para construir testes exatos e ótimos. Já para o caso desbalanceado não existe uma tabela única para análise de variância, pois a partição da soma total de quadrados pode ser feita de várias maneiras (KHURI et al., 1998). Assim, a construção de um teste estatístico exato pode ser difícil e aproximações precisam ser empregadas. Para se testar hipóteses sobre os parâmetros de efeito fixo Verbeke e Molenberghs (2000) sugerem a utilização dos testes $t$ e Wald- $F$, os quais diferem dos procedimentos padrões da ANOVA.

Quando se utiliza os testes $t$ e Wald- $F$, deve-se determinar o número aproximado de graus de liberdade $(\nu)$ para as distribuições $t$ e $F$. Na distribuição $F$ tem-se que o número de graus de liberdade do numerador é igual ao número de contrastes que serão testados, mas o número de graus de liberdade do denominador precisa ser calculado de maneira aproximada. Existem vários métodos para aproximação do número de graus de liberdade, dentre os quais pode-se citar: o método Residual, o método Containment, que é o método padrão do software SAS (SAS INSTITUTE, 2004), o método de Satterthwaite (1941) e o método de Kenward-Roger (KENWARD; ROGER, 1997).

Ao realizar um experimento com dados desbalanceados o pesquisador pode se deparar com a necessidade de escolher um desses métodos para aproximação do número de graus de liberdade, surge a preocupação em escolher o método mais adequado para prosseguir com sua análise de maneira mais eficiente possível. No presente trabalho foram realizadas simulações com diferentes cenários experimentais, utilizando-se o delineamento casualizado em blocos em uma situação e o esquema de tratamentos em parcelas subdividas em outra, com o intuito de comparar os métodos Containment, Residual, Satterthwaite e Kenward-Roger. Como critério de comparação entre os métodos utilizou-se o poder analítico e o poder simulado do teste Wald- $F$ e a taxa de erro tipo I. 


\section{REVISÃO BIBLIOGRÁFICA}

Nesta seção são apresentados aspectos teóricos relacionados aos estudos que serão abordados nesta dissertação.

\section{$2.1 \quad$ Teste de Hipóteses}

Segundo Andrade e Ogliari (2013), em muitas situações práticas o interesse do pesquisador é verificar a veracidade de uma suposição ou afirmação sobre um ou mais parâmetros ou sobre a distribuição de uma variável aleatória, podendo esta suposição ser verdadeira ou não. Essas afirmações são consideradas hipóteses estatísticas, porque se referem a parâmetros ou distribuições, e comumente precisam ser testadas a partir de amostras. A construção de uma teste de hipótese requer a especificação de duas hipóteses, denominadas de hipótese nula $\left(H_{0}\right)$ e hipótese alternativa $\left(H_{1}\right)$, e de um critério para a rejeição da hipótese nula.

Por exemplo, se um experimentador deseja comparar as produções médias de duas cultivares de soja, representadas por $\mu_{1}$ e $\mu_{2}$, as hipóteses nula e alternativa poderiam ser representadas respectivamente por: $H_{0}: \mu_{1}=\mu_{2}$ e $H_{1}: \mu_{1} \neq \mu_{2}$.

Em um problema envolvendo um teste de hipótese, depois de observar os resultados amostrais, o experimentador deve decidir se aceita $H_{0}$ como verdadeira ou se a rejeita, considerando $H_{1}$ como verdadeira. Casella e Berger (2002) determinam que um procedimento para testar uma hipótese é uma regra que especifica:

i) Para quais valores amostrais a decisão $H_{0}$ é aceita como verdadeira.

ii) Para quais valores amostrais $H_{0}$ é rejeitada e $H_{1}$ é aceita como verdadeira.

Definidos o teste estatístico e uma regra de decisão, determina-se o valor crítico da estatística e a um nível de significância $\alpha$ identifica-se a região crítica do teste ou região de rejeição de $H_{0}$, em que a região crítica é o subconjunto do espaço amostral em que $H_{0}$ será rejeitada, e a região de aceitação é o complemento da região de rejeição. Se a hipótese nula for rejeitada, conclui-se que a evidência favorece a hipótese alternativa, caso contrário, conclui-se que não há provas suficientes para rejeitar a hipótese nula. 


\subsubsection{Erros tipo I e tipo II}

Ao decidir quanto a aceitar ou rejeitar a hipótese nula $H_{0}$, o experimentador pode estar cometendo um erro. Comumente, testes de hipótese são avaliados e comparados por meio de suas probabilidades de resultar em erros. Ao se tomar uma decisão, a favor ou contra uma hipótese, existem dois tipos de erros que se pode cometer. Pode-se rejeitar uma hipótese nula quando de fato ela é verdadeira (erro Tipo I) ou pode-se não-rejeitar $H_{0}$ quando, de fato, ela é falsa (erro Tipo II). Frequentemente, denotam-se as probabilidades de ocorrência destes dois tipos de erros como $\alpha$ e $\beta$, respectivamente. Pode-se resumir os tipos de decisão e erros associados a testes de hipóteses da maneira apresentada na Tabela 1.

Tabela 1 - Possíveis situações no teste de hipótese

\begin{tabular}{ccc}
\hline & \multicolumn{2}{c}{$H_{0}$} \\
\cline { 2 - 3 } Decisão & Verdadeira & Falsa \\
\hline Aceitar $H_{0}$ & Decisão correta $(1-\alpha)$ & Erro tipo II $(\beta)$ \\
Rejeitar $H_{0}$ & Erro tipo I $(\alpha)$ & Decisão correta $(1-\beta)$ \\
\hline
\end{tabular}

A probabilidade de aceitar $H_{0}$ dado que $H_{0}$ é verdadeira é $(1-\alpha)$ e a probabilidade de ocorrência do erro tipo I é $P\left(\right.$ Rejeitar $H_{0} \mid H_{0}$ é verdadeira $)=\alpha$. A probabilidade de rejeitar $H_{0}$ dado que $H_{0}$ é falsa é $(1-\beta)$ e a probabilidade de ocorrência do erro tipo II é $P\left(\right.$ Aceitar $H_{0} \mid H_{0}$ é falsa $)=\beta$. A situação ideal em um teste de hipótese é aquela em que estas probabilidades são relativamente "baixas", o que, em geral, nem sempre ocorre. Segundo Cousens e Marshall (1987), é relevante salientar que os pesquisadores que observam em seus experimentos falta de efeito de tratamento devem incorporar em suas interpretações a possibilidade de cometer o erro tipo II. Ao fixar o nível de significância $\alpha$ do teste, o pesquisador está controlando apenas a probabilidade de ocorrência do erro tipo I, não do erro tipo II.

\subsubsection{Poder de um teste de hipótese}

Poder de um teste estatístico é a probabilidade $(1-\beta)$ de rejeitar a hipótese nula $H_{0}$ quando ela é falsa. Segundo Berndtson (1991) o poder de um teste estatístico é a probabilidade de que um efeito de tratamento não passe despercebido, caso exista tal efeito. Segundo Cohen (1988) o poder de um teste estatístico depende de três aspectos: 
- Do nível de significância $(\alpha)$ que representa o risco de engano de rejeitar a hipótese nula, ou ainda, a taxa de rejeição de uma hipótese nula verdadeira. Quanto menor o seu valor, mais rigoroso o nível de rejeição da hipótese nula. Porém, quando se diminui o nível de significância se aumenta a região de aceitação da hipótese nula e em consequência se aumenta a probabilidade de aceitar a hipótese nula quando ela é falsa, isto é, aumenta a probabilidade de cometer um erro tipo II, reduzindo desta forma o poder do teste.

- Da confiabilidade de um valor da amostra que está relacionada com a proximidade da estimativa de um parâmetro ao seu verdadeiro valor, que geralmente é desconhecido. Dependendo da estatística em questão e do modelo estatístico específico em que o teste é construído, a confiabilidade da estimativa do parâmetro pode ou não depender da unidade de medição, do valor do parâmetro e da forma da distribuição da população, porém a confiabilidade é sempre dependente do tamanho da amostra. Comumente o erro padrão é utilizado para avaliar a confiabilidade de uma estatística. Quanto menor o erro padrão maior é a confiabilidade dos resultados e maior o poder do teste. Uma das formas de diminuir o erro padrão é aumentar o tamanho da amostra, mantendo fixo os outros fatores envolvidos. Dessa forma conclui-se que quanto maior o tamanho da amostra maior será o poder do teste.

- Do tamanho do efeito que indica o grau com que o fenômeno está presente na população, ou ainda, o grau em que a hipótese nula é falsa. Qualquer que seja a forma de representação de um fenômeno em uma investigação particular de tratamentos, a hipótese nula significa sempre que o tamanho do efeito é zero. Para calcular o tamanho do efeito precisa-se de um número puro ou adimensional para o índice do grau de desvio, ou seja, um número livre da unidade de medida original. Por exemplo, para calcular o tamanho do efeito entre duas médias de tratamentos, usa-se,

- para o caso unicaudal:

$$
d=\frac{\mu_{A}-\mu_{B}}{\sigma}
$$

- para o caso bicaudal:

$$
d=\frac{\left|\mu_{A}-\mu_{B}\right|}{\sigma}
$$


onde, $d$ representa o índice do tamanho do efeito, $\mu_{A}$ e $\mu_{B}$ são as médias dos tratamentos e $\sigma$ é o desvio padrão comum das populações envolvidas.

Foram descritas quatro características de inferência estatística: poder do teste $(1-\beta)$, nível de significância $(\alpha)$, tamanho da amostra $(n)$ e tamanho do efeito, que estão relacionadas entre si, ou seja, qualquer uma delas é determinada como uma função das outras três (COHEN, 1965).

\subsection{Modelos lineares mistos}

É considerado um modelo misto aquele que contém fatores de efeitos fixos além da constante $\mu$ e fatores de efeitos aleatórios além do erro experimental.

De uma maneira geral, segundo Searle (1971), Henderson (1990) dentre outros, o modelo misto pode ser denotado por:

$$
\mathbf{y}=\mathbf{X} \boldsymbol{\beta}+\mathbf{Z u}+\boldsymbol{\epsilon}
$$

em que y é o vetor $n \times 1$ de observações da variável resposta $Y$ para $n$ indivíduos; $\mathbf{X}$ é a matriz $n \times p$ de incidência dos efeitos fixos, conhecida; $\boldsymbol{\beta}$ é o vetor $p \times 1$ dos efeitos fixos; $\mathbf{Z}$ é a matriz $n \times q$ de incidência dos efeitos aleatórios, conhecida; $\mathbf{u}$ é o vetor $q \times 1$ dos efeitos aleatórios e $\boldsymbol{\epsilon}$ é o vetor $n \times 1$ de erros aleatórios. Sendo $n$ o número de observações, $p$ o número de parâmetros de efeito fixo e $q$ o número de efeitos aleatórios.

Assume-se que $\boldsymbol{\epsilon}$ tem distribuição normal multivariada com vetor de média $\mathbf{0}$ e matriz de variâncias e covariâncias $\Sigma$, de dimensão $(n \times n)$. O vetor de efeitos aleatórios $\mathbf{u}$ tem distribuição normal multivariada com vetor de média $\mathbf{0}$ e matriz de variâncias e covariâncias $\mathbf{G}$, de dimensão $(q \times q)$. Tem-se, então,

$$
\mathrm{E}(\boldsymbol{Y})=\boldsymbol{X} \boldsymbol{\beta} \quad \text { e } \quad \operatorname{Var}(\boldsymbol{Y})=\boldsymbol{V}=\boldsymbol{\Sigma}+\boldsymbol{Z} \boldsymbol{G} \boldsymbol{Z}^{\prime}
$$

As características mais desejadas na obtenção do estimador do vetor de parâmetros de efeito fixo e do preditor do vetor de efeito aleatório, quando a matriz de variâncias e covariâncias $\boldsymbol{V}$ é conhecida, são o BLUE (best linear unbiased estimator "melhor estimador linear não viesado") e o BLUP (best linear unbiased predictor - "melhor preditor linear não viesado"), respectivamente.

Assim, se $\boldsymbol{G}$ e $\boldsymbol{\Sigma}$ são conhecidas e portanto $\mathbf{V}$ é conhecida, tem-se o melhor estimador linear não viesado (BLUE) de $\boldsymbol{\beta}$ e o melhor preditor linear não viesado (BLUP) 
de $\boldsymbol{u}$, que são obtidos respectivamente por,

$$
\hat{\boldsymbol{\beta}}=\left(\boldsymbol{X}^{\prime} \boldsymbol{V}^{-1} \boldsymbol{X}\right)^{-} \boldsymbol{X}^{\prime} \boldsymbol{V}^{-1} \boldsymbol{y}
$$

e

$$
\hat{\boldsymbol{u}}=\boldsymbol{G} \boldsymbol{Z}^{\prime} \boldsymbol{V}^{-1}(\boldsymbol{y}-\boldsymbol{X} \hat{\boldsymbol{\beta}}),
$$

Quando $\mathbf{G}$ e $\boldsymbol{\Sigma}$ não são conhecidas, o que ocorre na maioria das aplicações práticas, os componentes de variância a eles associados podem ser estimados eficientemente empregando-se o método de estimação REML (PATTERSON; THOMPSON, 1971; SEARLE et al., 1992). Nesse caso a predição dos efeitos aleatórios é obtida pelo BLUP empírico (E-BLUP) e as estimativas dos efeitos fixos são obtidas pelo BLUE empírico (EBLUE) que geralmente não tem as propriedades do BLUE, exceto em algumas situações de dados balanceados. Especificamente, estas estimativas não são funções lineares dos dados observados e não têm a propriedade de variância mínima (HENDERSON, 1963; KACKAR; HARVILLE, 1981; 1984).

\subsubsection{Estruturas da matriz de variâncias e covariâncias}

Algumas estruturas da matriz de variâncias e covariâncias são apresentadas a seguir:

1. Componente de Variância (CV):

Essa estrutura assume que não existe correlação entre qualquer par de observações. Essa matriz é utilizada geralmente como estrutura para $\boldsymbol{\Sigma}$ nos modelos ANOVA (todos os fatores são tratados como qualitativos, modelo padrão fixo), e contém apenas um parâmetro. Essa estrutura supõe independência e homogeneidade de variâncias entre os componentes.

$$
\left[\begin{array}{cccc}
\sigma^{2} & 0 & \cdots & 0 \\
0 & \sigma^{2} & \cdots & 0 \\
\vdots & \vdots & \ddots & \vdots \\
0 & 0 & \cdots & \sigma^{2}
\end{array}\right]
$$


2. Simetria Composta (SC):

A estrutura SC admite homogeneidade de variâncias e covariâncias constantes, é apropriada somente quando a condição Huynh-Feldt (condição de esfericidade) é cumprida, ou seja, é necessário uma igual correlação entre medidas no mesmo indivíduo (HUYNH; FELDT, 1970; 1976). Se essa condição não é satisfeita, então, outra estrutura de covariância deve ser utilizada. Esse tipo de estrutura pode ser visto em experimentos casualizados em blocos, em que o efeito de bloco é aleatório. A estrutura SC tem dois parâmetros $\left(\sigma_{1}^{2}\right.$ e $\left.\sigma^{2}\right)$.

$$
\left[\begin{array}{cccc}
\sigma_{1}^{2}+\sigma^{2} & \sigma_{1}^{2} & \cdots & \sigma_{1}^{2} \\
\sigma_{1}^{2} & \sigma_{1}^{2}+\sigma^{2} & \cdots & \sigma_{1}^{2} \\
\vdots & \vdots & \ddots & \vdots \\
\sigma_{1}^{2} & \sigma_{1}^{2} & \cdots & \sigma_{1}^{2}+\sigma^{2}
\end{array}\right]
$$

3. Simetria Composta Heterogênea $\left(S C_{H}\right)$ :

A estrutura $S C_{H}$ pressupõe variâncias diferentes para cada elemento da diagonal principal, covariâncias distintas e correlações iguais. Requer $t+1$ parâmetros estimados.

$$
\left[\begin{array}{cccc}
\sigma_{1}^{2} & \sigma_{1} \sigma_{2} \rho & \cdots & \sigma_{1} \sigma_{t} \rho \\
\sigma_{2} \sigma_{1} \rho & \sigma_{2}^{2} & \cdots & \sigma_{2} \sigma_{t} \rho \\
\vdots & \vdots & \ddots & \vdots \\
\sigma_{t} \sigma_{1} \rho & \sigma_{t} \sigma_{2} \rho & \cdots & \sigma_{t}^{2}
\end{array}\right]
$$

\subsubsection{Estimação dos componentes de variância}

$\mathrm{Na}$ análise de dados desbalanceados utilizando modelos lineares mistos a estimação dos componentes de variância tem importância extrema e depende da estrutura de matriz de variâncias e covariâncias escolhida e do método de estimação utilizado na estimação dos seus componentes. Existem diferentes métodos de estimação que podem levar a diferentes estimativas de um mesmo parâmetro. Dada a importância do tema, muitos métodos têm sido propostos para a estimação dos componentes de variância dentre os quais destacam-se: o método de Fisher (1918), o método da análise de variância 
(ANOVA), os métodos I, II e III de Henderson (1953), o método da máxima verossimilhança (ML) de Hartley e Rao (1967), o método da máxima verossimilhança restrita (REML) de Patterson e Thompson (1971), o método do estimador quadrático não-viesado de norma mínima (MINQUE) de Rao (1971a), o método do estimador quadrático nãoviesado de variância mínima (MIVQUE) de Rao (1971b) e o método iterativo do estimador quadrático não-viesado de norma mínima (I-MINQUE) de Searle (1987). No presente trabalho será apresentado uma breve descrição de dois desses métodos: Máxima Verossimilhança (ML) e Máxima Verossimilhança Restrita (REML).

\subsubsection{Método da Máxima Verossimilhança}

O método de estimação por máxima verossimilhança (ML) foi desenvolvido por Fisher (1925) e aplicado pela primeira vez em modelos mistos por Hartley e Rao (1967). Este método consiste em maximizar a função de verossimilhança das observações em relação aos efeitos fixos e aos componentes de variância, geralmente assumindo a suposição de normalidade dos dados.

Segundo Searle et al. (1992) a utilização do método da máxima verossimilhança (ML) para estimar os componentes de variância, deu-se sob a suposição de normalidade, considerando dados balanceados ou desbalanceados. Para os dados desbalanceados os métodos iterativos tornaram-se necessários.

As estimativas obtidas são sempre não negativas mas viesadas, por não considerar a perda do número de graus de liberdade resultante da estimação dos efeitos fixos do modelo. Deste modo, esse método tende a subestimar os componentes de variância.

\subsubsection{Método da Máxima Verossimilhança Restrita}

O método da máxima verossimilhança restrita (REML) foi desenvolvido para modelos balanceados específicos por Anderson e Bancroft (1952) e Russell e Bradley (1958). Thompson (1962) estendeu o método para todos os modelos balanceados, mas foram Patterson e Thompson (1971) que desenvolveram o método REML como tem sido utilizado atualmente, considerando-se a distribuição normal (HARVILLE, 1977). Este método tem sido amplamente adotado para a estimação dos componentes de variâncias da matriz V (THOMPSON 1980; ROBINSON 1991). O REML é um método iterativo e exige normalidade dos dados, como o método da ML. No entanto, o REML considera a 
perda dos graus de liberdade envolvidos na estimação dos parâmetros de efeitos fixos do modelo, fornecendo estimativas menos viesadas que as estimativas de ML (HARVILLE, 1977 e GILMOUR; THOMPSON; CULLIS, 1995).

Em experimentos com dados balanceados, as soluções fornecidas pelo método REML a partir de modelos mistos coincidem com as soluções fornecidas pelo método da ANOVA (SEARLE; CASELLA; MCCULLOCH, 1992). Já para dados desbalanceados, ambos os métodos ML e REML, fornecem estimativas viesadas. Porém, dadas as propriedades das estimativas obtidas pelo método REML, ele tem sido preferido quando os dados são desbalanceados (SEARLE 1971; HARVILLE 1977; e PERRI \& IEMMA 1999). Para maiores detalhes a respeito do método REML pode ser consultado o trabalho de Alcarde (2012), o qual é apresentada a base das deduções do método.

\subsection{Testes para os efeitos fixos}

Segundo Khuri et al., (1998) a construção de um teste estatístico exato para experimentos com dados desbalanceados pode ser difícil e aproximações precisam ser empregadas. Para se testar hipóteses sobre os parâmetros de efeito fixo Verbeke e Molenberghs (2000) sugerem a utilização dos testes $t$ e Wald-F.

\subsubsection{Teste aproximado $t$}

Para a estatística do teste $t$ considere o modelo (1) conforme descrito na seção 2.2 e um vetor $\boldsymbol{h}, p \times 1$, com $\operatorname{posto}(\boldsymbol{h})=1$, em que a hipótese nula testada é da forma:

$$
H_{0}: \boldsymbol{h}^{\prime} \boldsymbol{\beta}=0
$$

A estatística de teste é dada por:

$$
t=\frac{\boldsymbol{h}^{\prime} \hat{\boldsymbol{\beta}}}{\sqrt{\boldsymbol{h}^{\prime}\left(\boldsymbol{X}^{\prime} \hat{\boldsymbol{V}}^{-1} \boldsymbol{X}\right)^{-} \boldsymbol{h}}}
$$

Em geral, para o contexto dos modelos mistos, a estatística de teste tem uma distribuição aproximada $t \operatorname{com} \nu$ graus de liberdade, em que o número aproximado de graus de liberdade pode ser calculado utilizando um dos vários métodos existentes, como: Residual, Containment, Satterthwaite e Kenward-Roger. 


\subsubsection{Teste aproximado Wald- $F$}

Para a estatística do teste Wald- $F$ considere o modelo (1) conforme descrito na seção 2.2 e uma matriz de contrastes estimáveis $\boldsymbol{L}, p \times r, \operatorname{com} \operatorname{posto}(\boldsymbol{L})=l$, em que a hipótese nula testada é da forma:

$$
H_{0}: \boldsymbol{L}^{\prime} \boldsymbol{\beta}=0
$$

A estatística de teste para $\operatorname{posto}(\boldsymbol{L})>1$, é dada por:

$$
F=\frac{\hat{\boldsymbol{\beta}}^{\prime} \boldsymbol{L}\left(\boldsymbol{L}^{\prime}\left(\boldsymbol{X}^{\prime} \hat{\boldsymbol{V}}^{-1} \boldsymbol{X}\right)^{-} \boldsymbol{L}\right)^{-1} \boldsymbol{L}^{\prime} \hat{\boldsymbol{\beta}}}{\operatorname{posto}(\boldsymbol{L})}
$$

Em geral, a estatística de teste tem uma distribuição aproximada $F$ com o número de graus de liberdade do numerador dado pelo posto(L) e o número aproximado de graus de liberdade $\nu$ do denominador é calculado por métodos existentes, como: Residual, Containment, Satterthwaite e Kenward-Roger.

\subsection{Métodos para aproximar o número de graus de liberdade}

Existem vários métodos para aproximar o número de graus de liberdade $\nu$ nos testes $t$ e Wald- $F$ no modelo (1), dos quais considerou-se:

1. Método Residual

O número aproximado de graus de liberdade $\nu$ é dado por:

$$
\nu=n-\operatorname{posto}(\mathbf{X})
$$

2. Método Containment

O número aproximado de graus de liberdade $\nu$ é dado por:

$$
\nu=n-\operatorname{posto}(\mathbf{X}, \mathbf{Z})
$$

3. Método de Satterthwaite

Este método propõe uma aproximação de uma distribuição exata, usando para isso uma distribuição $\chi^{2}$ na qual o número de graus de liberdade é escolhido de modo a proporcionar uma boa concordância entre as duas distribuições. Consegue-se isso 
encontrando uma aproximação de $\chi^{2}$ que tenha uma variação igual a da distribuição exata (SATTERTHWAITE, 1946).

A fim de aproximar o teste $F$ para os efeitos fixos em um modelo como descrito em (1), o método de Satterthwaite usa a estatística tipo Wald,

$$
F=\frac{1}{l} \hat{\boldsymbol{\beta}}^{\prime} \boldsymbol{L}\left(\boldsymbol{L}^{\prime} \hat{\boldsymbol{\Phi}} \boldsymbol{L}\right)^{-1} \boldsymbol{L}^{\prime} \hat{\boldsymbol{\beta}}
$$

Em geral, a estatística de teste tem uma distribuição aproximada $F(l, \nu)$, onde $l=\operatorname{posto}(\boldsymbol{L})$, isto é, $l$ é o número de combinações lineares independentes de $\boldsymbol{\beta}$ em $\boldsymbol{L}, \nu$ é o numero aproximado de graus de liberdade associado com $\boldsymbol{L}^{\prime}\left(\boldsymbol{X}^{\prime} \hat{\boldsymbol{V}}^{-1} \boldsymbol{X}\right) \boldsymbol{L}$ e $\hat{\boldsymbol{\Phi}}=\left(\boldsymbol{X}^{\prime} \hat{\boldsymbol{V}}^{-1} \boldsymbol{X}\right)^{-}$é a matriz estimada de covariâncias de $\hat{\boldsymbol{\beta}}$. A aproximação do número de graus de liberdade (SAS INSTITUTE, 2013) é computado da seguinte forma:

i) Caso multidimensional, ou seja, $\boldsymbol{L}$ é uma matriz de contrastes estimáveis de posto $l>1$, neste caso realiza-se primeiramente a decomposição espectral:

$$
L^{\prime} \hat{\Phi} L=P^{\prime} D P
$$

em que $\boldsymbol{P}$ é uma matriz ortogonal de autovetores e $\boldsymbol{D}$ é uma matriz diagonal de autovalores, ambos de dimensão $l \times l$. Seja,

$$
\nu_{m}=\frac{2\left(d_{m}\right)^{2}}{g_{m}^{\prime} \boldsymbol{W} g_{m}}
$$

em que, $d_{m}$ é o $m$-ésimo elemento da diagonal de $\boldsymbol{D}, \boldsymbol{W}$ é a matriz assintótica de variâncias e covariâncias de $\hat{\boldsymbol{\theta}}$, que é a estimativa do vetor dos componentes de variâncias, $\boldsymbol{\theta}$, e $g_{m}$ é o vetor gradiente de $a_{m} \boldsymbol{\Phi} a_{m}^{\prime}$ em relação a $\boldsymbol{\theta}$ avaliada para $\hat{\boldsymbol{\theta}}$, onde $a_{m}$ é a $m$-ésima linha de $\boldsymbol{P} \boldsymbol{L}^{\prime}$. Note que,

$$
g_{m}=\left[\cdots \frac{\partial\left(a_{m} \boldsymbol{\Phi} a_{m}^{\prime}\right)}{\partial \theta_{i}} \cdots\right]_{\boldsymbol{\theta}=\hat{\boldsymbol{\theta}}}=-\left[\cdots a_{m} \boldsymbol{\Phi} \boldsymbol{P}_{i} \boldsymbol{\Phi} a_{m}^{\prime} \cdots\right]_{\boldsymbol{\theta}=\hat{\boldsymbol{\theta}}}
$$

é o vetor de derivadas com respeito a cada um dos componentes de variâncias. Considere,

$$
E=\sum_{m=1}^{l} \frac{\nu_{m}}{\nu_{m}-2} \quad \text { para } \quad \nu_{m}>2
$$

Assim, eliminando os termos para $\nu_{m} \leq 2$, o número de graus de liberdade para $F$ é dado por:

$$
\nu=\left\{\begin{array}{l}
\frac{2 E}{E-l}, \quad \text { para } E>l \\
0, \quad \text { caso contrário }
\end{array}\right.
$$


ii) Caso uni-dimensional, ou seja, $\operatorname{posto}(\boldsymbol{L})=1$. Neste caso, a estatística $F$ é dada por

$$
F=\frac{\left(\boldsymbol{L}^{\prime} \hat{\boldsymbol{\beta}}\right)^{2}}{\boldsymbol{L}^{\prime} \boldsymbol{\Phi} \boldsymbol{L}}
$$

Note que também pode ser usado a estatística $t$, desde que o número de graus de liberdade do numerador seja igual a 1. O número do grau de liberdade do denominador é dado por:

$$
\nu=\frac{2\left(\boldsymbol{L}^{\prime} \hat{\boldsymbol{\Phi}} \boldsymbol{L}\right)^{2}}{\boldsymbol{g}^{\prime} \boldsymbol{W} \boldsymbol{g}}
$$

onde $\boldsymbol{g}$ é o vetor gradiente de $\boldsymbol{L}^{\prime} \hat{\boldsymbol{\Phi}} \boldsymbol{L}$ em relação a $\boldsymbol{\theta}$ (assumindo que $\boldsymbol{L}^{\prime} \boldsymbol{\beta}$ é estimável).

4. Método de Kenward-Roger

Considere o modelo (1) conforme descrito na seção 2.2, em que as suposições com relação às variáveis aleatórias, $\boldsymbol{Y}$ e $\boldsymbol{u}$, são dadas por:

$$
\begin{gathered}
\boldsymbol{Y} \mid \boldsymbol{u} \sim N(\boldsymbol{X} \boldsymbol{\beta}+\boldsymbol{Z} \boldsymbol{u}, \boldsymbol{\Sigma}) \\
\boldsymbol{u} \sim N(\mathbf{0}, \boldsymbol{G})
\end{gathered}
$$

E marginalmente, $\boldsymbol{Y} \sim N(\boldsymbol{X} \boldsymbol{\beta}, \boldsymbol{V})$, em que $\boldsymbol{V}=\boldsymbol{Z} \boldsymbol{G} \boldsymbol{Z}^{\prime}+\boldsymbol{\Sigma}$.

O estimador de máxima verossimilhança restrita (REML) do vetor de componentes de variâncias $\boldsymbol{\theta}$, denotado por $\hat{\boldsymbol{\theta}}$, baseado no estimador de mínimos quadrados generalizado de $\boldsymbol{\beta}$ é:

$$
\hat{\boldsymbol{\beta}}=\left[\boldsymbol{X}^{\prime} \boldsymbol{V}^{-1}(\hat{\boldsymbol{\theta}}) \boldsymbol{X}\right]^{-1} \boldsymbol{X}^{\prime} \boldsymbol{V}^{-1}(\hat{\boldsymbol{\theta}}) \boldsymbol{Y}
$$

Usualmente, a precisão de $\hat{\boldsymbol{\beta}}$ é obtida a partir de uma estimativa da matriz de variâncias e covariâncias, $\boldsymbol{V}$. No entanto a estimativa de $\boldsymbol{\beta}$ não leva em conta a variabilidade na estimativa de $\boldsymbol{V}$, que para certas combinações de estrutura de covariâncias, delineamentos e tamanho de amostras, pode ter um impacto significativo sobre a precisão de $\boldsymbol{\beta}$ (KENWARD; ROGER, 1997). Além disso, os procedimentos do teste tipo Wald e intervalos de confiança correspondentes que são construídos com base em aproximações de uma distribuição $\chi^{2}$ assintótica, também ignoram a variabilidade na estimativa de $\boldsymbol{V}$, ou seja, o estimador convencional da matriz 
de variâncias e covariâncias subestima a estimativa dos efeitos fixos (KACKAR; HARVILLE, 1984).

O problema da precisão de $\hat{\boldsymbol{\beta}}$ para pequenas amostras já foi exaustivamente explorado em uma série de artigos escritos por Harville e colaboradores (JESKE; HARVILLE, 1988; HULTING; HARVILLE, 1991; HARVILLE; JESKE, 1992; HARVILLE; CARRIQUIRY, 1992).

Kackar e Harville (1984) mostraram que a variabilidade de $\hat{\boldsymbol{\beta}}$ pode ser particionada em 2 componentes,

$$
\operatorname{Var}(\hat{\boldsymbol{\beta}})=\boldsymbol{\Phi}+\boldsymbol{\Lambda}
$$

em que o primeiro componente é a matriz de variâncias e covariâncias do estimador de efeitos fixos, e o segundo componente representa o valor que o primeiro componente subestima. A estimativa do primeiro componente foi abordada por Harville e Jeske (1992) e o segundo componente foi aproximado por Kackar e Harville (1984) e Prasad e Rao (1990). Em 1997, Kenward e Roger usaram os estimadores combinados dos dois componentes para produzir um estimador ajustado para a matriz de variâncias e covariâncias de $\hat{\boldsymbol{\beta}}$.

As demonstrações das expressões utilizadas a seguir podem ser encontradas em Alnosaier (2007). Usando uma expansão da série de Taylor sobre $\boldsymbol{\theta}$, Kackar e Harville (1984) mostraram que $\boldsymbol{\Lambda}$ pode ser aproximada por:

$$
\boldsymbol{\Lambda} \cong \boldsymbol{\Phi}\left[\sum_{i=1}^{d} \sum_{j=1}^{d} W_{i j}\left(\boldsymbol{Q}_{i j}-\boldsymbol{D}_{i} \boldsymbol{\Phi} \boldsymbol{D}_{j}\right)\right] \boldsymbol{\Phi}
$$

em que,

$$
\boldsymbol{D}_{i}=\boldsymbol{X}^{\prime} \frac{\partial \boldsymbol{V}^{-1}}{\partial \theta_{i}} \boldsymbol{X} \quad \text { e } \quad \boldsymbol{Q}_{i j}=\boldsymbol{X}^{\prime} \frac{\partial \boldsymbol{V}^{-1}}{\partial \theta_{i}} \boldsymbol{V} \frac{\partial \boldsymbol{V}^{-1}}{\partial \theta_{j}} \boldsymbol{X}
$$

e $W_{i j}$ são elementos de $\boldsymbol{W}=\operatorname{Var}(\hat{\boldsymbol{\theta}})$

Considerando o viés em $\hat{\boldsymbol{\Phi}}$ como um estimador de $\boldsymbol{\Phi}$ e uma expansão da série de Taylor sobre $\boldsymbol{\theta}$ tem-se:

$$
\hat{\boldsymbol{\Phi}} \cong \boldsymbol{\Phi}+\sum_{i=1}^{d}\left(\hat{\theta}_{i}-\theta_{i}\right) \frac{\partial \boldsymbol{\Phi}}{\partial \theta_{i}}+\frac{1}{2} \sum_{i=1}^{d} \sum_{j=1}^{d}\left(\hat{\theta}_{i}-\theta_{i}\right)\left(\hat{\theta}_{j}-\theta_{j}\right) \frac{\partial^{2} \boldsymbol{\Phi}}{\partial \theta_{i} \partial \theta_{j}}
$$

Ignorando um possível viés em $\hat{\boldsymbol{\theta}}$ tem-se:

$$
\mathrm{E}(\hat{\mathbf{\Phi}}) \cong \boldsymbol{\Phi}+\frac{1}{2} \sum_{i=1}^{d} \sum_{j=1}^{d} W_{i j} \frac{\partial^{2} \boldsymbol{\Phi}}{\partial \theta_{i} \partial \theta_{j}}
$$


em que,

$$
\frac{\partial^{2} \boldsymbol{\Phi}}{\partial \theta_{i} \partial \theta_{j}}=\boldsymbol{\Phi}\left(\boldsymbol{D}_{i} \boldsymbol{\Phi} \boldsymbol{D}_{j}+\boldsymbol{D}_{j} \boldsymbol{\Phi} \boldsymbol{D}_{i}-\boldsymbol{Q}_{i j}-\boldsymbol{Q}_{j i}+\boldsymbol{R}_{i j}\right) \boldsymbol{\Phi}
$$

Sendo,

$$
\boldsymbol{R}_{i j}=\boldsymbol{X}^{\prime} \boldsymbol{V}^{-1} \frac{\partial^{2} \boldsymbol{V}}{\partial \theta_{i} \partial \theta_{j}} \boldsymbol{V}^{-1} \boldsymbol{X}
$$

Assim, combinando (6) e (7) obtém-se um estimador ajustado para a matriz de variâncias e covariâncias de $\hat{\boldsymbol{\beta}}$,

$$
\hat{\boldsymbol{\Phi}}_{A}=\hat{\boldsymbol{\Phi}}+2 \hat{\boldsymbol{\Phi}}\left\{\sum_{i=1}^{d} \sum_{j=1}^{d} W_{i j}\left(\boldsymbol{Q}_{i j}-\boldsymbol{D}_{i} \hat{\boldsymbol{\Phi}} \boldsymbol{D}_{j}-\frac{1}{4} \boldsymbol{R}_{i j}\right)\right\} \hat{\boldsymbol{\Phi}}
$$

Existe uma classe de estruturas de covariância definida pela forma linear

$$
\boldsymbol{V}=\sum_{i=1}^{d} \theta_{i} A_{i}=\theta_{1} \boldsymbol{A}_{\mathbf{1}}+\cdots+\theta_{d} \boldsymbol{A}_{\boldsymbol{d}}
$$

Em que $\boldsymbol{A}_{i}$ é uma matriz conhecida $n \times n$. Esta categoria inclui as matrizes de variâncias e covariâncias do tipo $\boldsymbol{V}=\boldsymbol{Z} \boldsymbol{G} \boldsymbol{Z}^{\prime}+\boldsymbol{R}$, decorrentes de modelos mistos. Para tais estruturas lineares de covariância

$$
\frac{\partial^{2} \boldsymbol{V}}{\partial \theta_{i} \partial \theta_{j}}=0
$$

O que indica que $R_{i j}=0$ na expressão (8), para todo par $(i, j)$. Simplificando a expressão (9) tem-se:

$$
\hat{\mathbf{\Phi}}_{A}=\hat{\boldsymbol{\Phi}}-\sum_{i=1}^{d} \sum_{j=1}^{d} W_{i j} \frac{\partial^{2} \boldsymbol{\Phi}}{\partial \theta_{i} \partial \theta_{j}}=\hat{\boldsymbol{\Phi}}+2 \hat{\boldsymbol{\Lambda}}
$$

para $\Lambda$ como representado em (6). Uma aproximação para a matriz de variâncias e covariâncias de $\hat{\boldsymbol{\theta}}, \boldsymbol{W}$, pode ser obtida a partir da inversa da matriz de informação esperada $I_{E}$, em que

$$
2 I_{E i j}=\operatorname{tr}\left(\frac{\partial \boldsymbol{V}^{-1}}{\partial \theta_{i}} \boldsymbol{V} \frac{\partial \boldsymbol{V}^{-1}}{\partial \theta_{j}} \boldsymbol{V}\right)-\operatorname{tr}\left(2 \boldsymbol{\Phi} \boldsymbol{Q}_{i j}-\boldsymbol{\Phi} \boldsymbol{D}_{i} \boldsymbol{\Phi} \boldsymbol{D}_{j}\right)
$$

Quando é necessário fazer inferências simultaneamente sobre as $l$ combinações lineares dos elementos de $\boldsymbol{\beta}$ utilizando $\boldsymbol{L} \boldsymbol{\beta}$, em que $\boldsymbol{L}$ é uma matriz de contrastes envolvendo os efeitos fixos $(p \times r)$ a proposta é utilizar a estimativa ajustada da 
matriz de variância e covariância, $\hat{\mathbf{\Phi}}_{A}$, na construção de uma estatística pivô tipo Wald, da seguinte forma:

$$
F=\frac{1}{l}(\hat{\boldsymbol{\beta}}-\boldsymbol{\beta})^{\prime} \boldsymbol{L}\left(\boldsymbol{L}^{\prime} \hat{\boldsymbol{\Phi}}_{A} \boldsymbol{L}\right)^{-1} \boldsymbol{L}^{\prime}(\hat{\boldsymbol{\beta}}-\boldsymbol{\beta})
$$

A derivação de uma aproximação de $F$ apropriada para (10) quando $l=1$ é comparável à aproximação utilizada por Satterthwaite (1941). Para $l>1$ a situação é mais complicada, pois é necessário levar em consideração a estrutura interna dos efeitos aleatórios de $\boldsymbol{L}^{\prime} \hat{\boldsymbol{\Phi}}_{A} \boldsymbol{L}$, e para cada contexto trabalhado essa estrutura pode ser diferente. Segundo (KRZANOWSKI, 2000) é conhecida, para a estatística $T^{2}$ de Hotelling, uma forma escalar de $F, F^{*}$, dada por

$$
F^{*}=\frac{\nu}{\nu+l-1} F
$$

que tem uma distribuição $F(l, \nu)$ exata.

Estudo com simulações tem mostrado que nas estatísticas da forma de $F$ em (10), se uma aproximação de $F$ com número de graus de liberdade do numerador igual a $l$ é usada, será necessário que $F^{*}=\lambda F$, em que $\lambda=\frac{\nu}{\nu+l-1}$ e normalmente $\lambda \leq 1($ KENWARD; ROGER, 1997).

Além disso, se a aproximação escolhida é para reproduzir os número de graus de liberdade corretos para a distribuição $F$ exata, então esse escalar terá que ser introduzido para acomodar a estatística $T^{2}$ de Hotelling (KRZANOWSKI, 2000). Para obter essa aproximação é necessário calcular dois valores a partir dos dados: o escalar $\lambda$ e o número de graus de liberdade do denominador $\nu$.

Utilizando uma expansão de série de Taylor para $\left(\boldsymbol{L}^{\prime} \hat{\boldsymbol{\Phi}}_{A} \boldsymbol{L}\right)^{-1}$ tem-se:

$\left(\boldsymbol{L}^{\prime} \hat{\boldsymbol{\Phi}}_{A} \boldsymbol{L}\right)^{-1} \cong\left(\boldsymbol{L}^{\prime} \boldsymbol{\Phi}_{A} \boldsymbol{L}\right)^{-1}+\sum_{i=1}^{d}\left(\hat{\theta}_{i}-\theta_{i}\right) \frac{\partial\left(\boldsymbol{L}^{\prime} \hat{\boldsymbol{\Phi}}_{A} \boldsymbol{L}\right)^{-1}}{\partial \theta_{i}}+\frac{1}{2} \sum_{i=1}^{d} \sum_{j=1}^{d}\left(\hat{\theta}_{i}-\theta_{i}\right)\left(\hat{\theta}_{j}-\theta_{j}\right) \frac{\partial^{2}\left(\boldsymbol{L}^{\prime} \hat{\boldsymbol{\Phi}}_{A} \boldsymbol{L}\right)^{-1}}{\partial \theta_{i} \partial \theta_{j}}$

Ignorando um possível viés em $\hat{\boldsymbol{\theta}}$ e uma possível dependência estatística entre $\hat{\boldsymbol{\beta}}$ e $\hat{\Phi}$ e usando as relações:

$$
\begin{aligned}
\mathrm{E}(F) & =\mathrm{E}_{\hat{\boldsymbol{\Phi}}_{A}}\left[\mathrm{E}\left[F \mid \hat{\boldsymbol{\Phi}}_{A}\right]\right] \\
\operatorname{Var}(F) & =\mathrm{E}_{\hat{\boldsymbol{\Phi}}_{A}}\left[\operatorname{Var}\left[F \mid \hat{\boldsymbol{\Phi}}_{A}\right]\right]+\operatorname{Var}_{\hat{\boldsymbol{\Phi}}_{A}}\left[\mathrm{E}\left[F \mid \hat{\boldsymbol{\Phi}}_{A}\right]\right]
\end{aligned}
$$


Depois de tomar as esperanças apropriadas e eliminar os termos de ordem superior na expansão de série de Taylor, pode-se escrever,

$$
\begin{aligned}
\mathrm{E}(F) & \cong 1+\frac{1}{l} A_{2} \\
\operatorname{Var}(F) & \cong \frac{2}{l}(1+B)
\end{aligned}
$$

sendo,

$$
B=\frac{1}{2 l}\left(A_{1}+6 A_{2}\right)
$$

em que

$$
\begin{aligned}
& A_{1}=\sum_{i=1}^{d} \sum_{j=1}^{d} W_{i j} \operatorname{tr}\left(\boldsymbol{\Theta} \boldsymbol{\Phi} \boldsymbol{D}_{i} \boldsymbol{\Phi}\right) \operatorname{tr}\left(\boldsymbol{\Theta} \boldsymbol{\Phi} \boldsymbol{D}_{j} \boldsymbol{\Phi}\right) \\
& A_{2}=\sum_{i=1}^{d} \sum_{j=1}^{d} W_{i j} \operatorname{tr}\left(\boldsymbol{\Theta} \boldsymbol{\Phi} \boldsymbol{D}_{i} \boldsymbol{\Phi} \Theta \boldsymbol{\Phi} \boldsymbol{D}_{j} \boldsymbol{\Phi}\right)
\end{aligned}
$$

para

$$
\Theta=\boldsymbol{L}\left(\boldsymbol{L}^{\prime} \Phi \boldsymbol{L}\right)^{-1} \boldsymbol{L}^{\prime}
$$

Deste modo, para uma determinada ordem de aproximação da série de Taylor nos correspondentes momentos de $F^{*}$ para a correspondente distribuição $F$ aproximada, tem-se,

$$
\nu=4+\frac{l+2}{l \rho-1}, \quad \text { onde } \quad \rho=\frac{\operatorname{Var}(F)}{2 \mathrm{E}(F)^{2}}
$$

$\mathrm{e}$

$$
\lambda=\frac{\nu}{\mathrm{E}(F)(\nu-2)}
$$

Para obter as estimativas exatas de $\nu$ e $\lambda$ modificam-se as suas estimativas, de modo que seja inalterada a aproximação até o fim da expansão da série de Taylor. De modo geral modifica-se a aproximação da esperança e variância de $F$ para produzir $\mathrm{E}^{*}(F)$ e $\operatorname{Var}^{*}(F)$, considerando,

$$
\begin{aligned}
\mathrm{E}(F) & =\mathrm{E}^{*}(F)=\left(1-\frac{A_{2}}{l}\right)^{-1} \\
\operatorname{Var}(F) & =\operatorname{Var}^{*}(F)=\frac{2}{l}\left[\frac{1+c_{1} B}{\left(1-c_{2} B\right)^{2}\left(1-c_{3} B\right)}\right]
\end{aligned}
$$


em que,

$$
\begin{aligned}
c_{1} & =\frac{g}{3 l+2(1-g)} \\
c_{2} & =\frac{1-g}{3 l+2(1-g)} \\
c_{3} & =\frac{l+2-g}{3 l+2(1-g)}
\end{aligned}
$$

para

$$
g=\frac{(l+1) A_{1}-(l+4) A_{2}}{(l+2) A_{2}}
$$

Substitui-se $E^{*}(F)$ e $\operatorname{Var}^{*}(F)$ nas expressões (11) e (12) para produzir as estimativas modificadas de $\nu$ e $\lambda$. Pode ser verificado que essas estimativas reproduzem os valores corretos quando $\lambda F$ tem uma distribuição $F$ exata (KENWARD; ROGER, 1997).

\subsection{Simulações computacionais}

Processos de simulação são comumente utilizados na área da Estatística, pois são uma forma eficiente de testar ou verificar a funcionalidade ou a eficiência de uma determinada metodologia. Nesse contexto, a simulação torna-se uma forma prática de geração de bancos de dados quando não há dados reais para que seja realizada uma determinada análise. Aplicações envolvendo simulações são adotadas em diversos estudos, como, por exemplo, na análise de regressão, quando se comparam os métodos clássico e bayesiano de estimação; na experimentação, quando há interesse em testar uma nova metodologia em cenários distintos. Por meio de alguns softwares, como o R e o SAS, que têm a capacidade de realizar simulação rápidas, pode-se realizar processos de simulação de acordo com as especificações do pesquisador.

Segundo Binder e Heermann (2010) os estudos com simulações rendem informação exata (além de erros estatísticos, que podem ser mantidos tão pequenos quanto desejado) para modelos que são precisamente caracterizados, enquanto que as informações fornecidas pela teoria analítica são exatas apenas em casos bastante raros, pois na maioria dos outros casos, são necessárias aproximações não controladas. Assim as simulações computacionais muitas vezes são projetadas para verificar a exatidão de alguma aproximação feita no tratamento analítico de um modelo. 


\section{MATERIAL E MÉTODOS}

\subsection{Modelos experimentais estudados}

Nessa seção serão apresentados os modelos experimentais utilizados para o processo de simulação, sendo considerado o delineamento casualizado em blocos e o delineamento casualizado em blocos com tratamentos no esquema de parcelas subdivididas. Também serão apresentados os cenários experimentais utilizados e a descrição do procedimento de simulação.

\subsubsection{Delineamento casualizado em blocos (DBC)}

O modelo utilizado para um experimento com um fator de tratamento planejado em um delineamento casualizado em blocos foi o seguinte,

$$
y_{i j}=\mu+\tau_{i}+\gamma_{j}+\epsilon_{i j}
$$

em que $i=1, \ldots, a ; j=1, \ldots, b ; \mu$ é uma constante inerente a todas observações; $\tau_{i}$ é o efeito do tratamento $i ; \gamma_{j}$ é o efeito do bloco $j$ e $\epsilon_{i j}$ é o erro experimental associado a $y_{i j}$. Foi considerado para esse modelo o efeito de tratamento $\tau_{i}$ fixo, isto é, $\mathrm{E}\left(\tau_{i}\right)=\tau_{i}$, o efeito de bloco $\gamma_{j}$ aleatório com distribuição normal com média zero e variância $\sigma_{B l}^{2}$, independente e identicamente distribuído e $\epsilon_{i j}$ como efeito aleatório, com distribuição normal com média zero e variância $\sigma_{R}^{2}$, independente e identicamente distribuído. Este delineamento é muito utilizado na realização de experimentos, pois leva em consideração os três princípios básicos da experimentação, repetição, casualização e controle local, sendo que o controle local é feito na sua forma mais simples e é chamado de blocos. Steel e Torrie (1981) afirmam que não há restrição para o número de tratamentos ou blocos a ser empregado neste delineamento, no entanto a experiência tem demonstrado que o número máximo de tratamentos é variável em função do tamanho de cada unidade experimental, da definição física de bloco e, muitas vezes, até da capacidade de trabalho da equipe envolvida ou da disponibilidade de recursos financeiros ou materiais (ZIMMERMANN, 2004). 


\subsubsection{Delineamento casualizado em blocos com tratamentos no esquema de parcelas subdivididas (DBC/PSD)}

O modelo utilizado foi o seguinte,

$$
y_{i j k}=\mu+\alpha_{i}+\beta_{j}+\gamma_{k}+(\alpha \beta)_{i j}+\varepsilon_{i k}+\epsilon_{i j k}
$$

em que $i=1, \ldots, a ; j=1, \ldots, b ; k=1, \ldots, c ; \mu$ é uma constante inerente a todas observações, $\alpha_{i}$ é o efeito do $i$-ésimo nível do fator $A$ aplicado à parcela, $\beta_{j}$ é o efeito do $j$-ésimo nível do fator $B$ aplicado à subparcela, $\gamma_{k}$ é o efeito do $k$-ésimo bloco, $(\alpha \beta)_{i j}$ é o efeito da interação $A B, \varepsilon_{i k}$ é o erro aleatório da parcela e $\epsilon_{i j k}$ é o erro experimental referente à subparcela ou associado a $y_{i j k}$. Segundo Zimmermann (2004) os experimentos em parcelas subdivididas podem ter origem no planejamento ou na forma de condução. No planejamento, os distintos fatores em estudo são divididos segundo algum critério, uns para constituírem as parcelas de tamanho maior, e outros as subparcelas de tamanho menor, que estarão contidas nas parcelas. Os níveis do fator principal são alocados às parcelas maiores segundo o delineamento experimental escolhido, e os níveis do outro fator são alocados ao acaso (por sorteio) em cada uma das subparcelas.

Em um primeiro momento foram considerados $\alpha_{i}$ e $\beta_{j}$ de efeitos fixos, isto é, $\mathrm{E}\left(\alpha_{i}\right)=\alpha_{i}$ e $\mathrm{E}\left(\beta_{j}\right)=\beta_{j}$. O efeito de bloco $\gamma_{k}$ foi considerado aleatório com distribuição normal de média zero e variância $\sigma_{B l}^{2}$, independente e identicamente distribuído. O erro da parcela $\varepsilon_{i k}$, aleatório, com distribuição normal com média zero, variância $\sigma_{P}^{2}$, independente e identicamente distribuído e $\epsilon_{i j k}$ aleatório com distribuição normal de média zero, variância $\sigma_{R}^{2}$, independente e identicamente distribuído.

Em um segundo momento considerou-se o efeito do fator $A, \alpha_{i}$, sendo de efeito aleatório com distribuição normal com média zero e variância $\sigma_{A}^{2}$, independente e identicamente distribuídos e as demais condições análogas às apresentadas anteriormente para esse modelo.

\subsection{Estrutura dos dados simulados}

Para as simulações dos experimentos casualizado em blocos com um fator de tratamento considerou-se as seguintes quantidades para tratamento e bloco, na devida ordem, (10, 3), (10, 6), (10, 12) para representar amostras de 30, 60 e 120 observações, respectivamente. Para cada amostra foi provocado um desbalanceamento no número de 
observações de forma aleatória considerando-se 3 níveis de desbalanceamento, 10\%, 20\% e $30 \%$ das observações e diferentes valores para $\sigma_{B l}^{2}(0,1 ; 0,5 ; 1,0 ; 5,0)$ com $\sigma_{R}^{2}$ fixo em uma unidade.

Para as simulações dos experimentos casualizado em blocos no esquema de parcelas subdivididas considerou-se as seguintes quantidades para os níveis do fator $A$, $B$ e bloco, na devida ordem, $(3,2,5),(3,4,5)$ e $(5,2,12)$ para representar amostras de 30, 60 e 120 observações, respectivamente. Para cada amostra foi provocado o desbalanceamento no número de observações de forma aleatória considerando-se 3 níveis de desbalanceamento, $10 \%, 20 \%$ e $30 \%$ das observações e diferentes valores para $\sigma_{B l}^{2}(0,1$; $0,5)$ e $\sigma_{P}^{2}(0,1 ; 0,5 ; 5 ; 1)$ com $\sigma_{R}^{2}$ fixo em uma unidade. Adicionalmente, considerou-se situações em que o efeito do fator $A, \alpha_{i}$, foi escolhido como aleatório, e os valores de $\sigma_{A}^{2}$ e $\sigma_{A B}^{2}$ foram fixados em 0,1 . A Tabela 2 mostra as especificações dos modelos que foram utilizados nas simulações realizadas.

Tabela 2 - Descrição dos modelos utilizados nas simulações

\begin{tabular}{ccccccc}
\hline Modelo & Delineamento & Fator Fixo & Fator Aleatório & $\mathrm{n}$ & $\begin{array}{c}\text { número } \\
\text { de blocos }\end{array}$ & $\begin{array}{c}\text { número de } \\
\text { tratamentos }\end{array}$ \\
\hline I & DBC & $\mu$ e $\tau_{i}$ & $\gamma_{j}$ e $\epsilon_{i j}$ & 30 & 3 & 10 \\
II & DBC & $\mu$ e $\tau_{i}$ & $\gamma_{j}$ e $\epsilon_{i j}$ & 60 & 6 & 10 \\
III & DBC & $\mu$ e $\tau_{i}$ & $\gamma_{j}$ e $\epsilon_{i j}$ & 120 & 12 & 10 \\
IV & DBC/PSD & $\mu, \alpha_{i}, \beta_{j}$ e $\alpha \beta_{i j}$ & $\gamma_{k}, \varepsilon_{i k}$ e $\epsilon_{i j k}$ & 30 & 5 & 6 \\
V & DBC/PSD & $\mu, \alpha_{i}, \beta_{j}$ e $\alpha \beta_{i j}$ & $\gamma_{k}, \varepsilon_{i k}$ e $\epsilon_{i j k}$ & 60 & 5 & 12 \\
VI & DBC/PSD & $\mu, \alpha_{i}, \beta_{j}$ e $\alpha \beta_{i j}$ & $\gamma_{k}, \varepsilon_{i k}$ e $\epsilon_{i j k}$ & 120 & 12 & 10 \\
VII & DBC/PSD & $\mu$ e $\beta_{j}$ & $\gamma_{k}, \alpha_{i}, \alpha \beta_{i j}, \varepsilon_{i k}$ e $\epsilon_{i j k}$ & 30 & 5 & 6 \\
VIII & DBC/PSD & $\mu$ e $\beta_{j}$ & $\gamma_{k}, \alpha_{i}, \alpha \beta_{i j}, \varepsilon_{i k}$ e $\epsilon_{i j k}$ & 60 & 5 & 12 \\
IX & DBC/PSD & $\mu$ e $\beta_{j}$ & $\gamma_{k}, \alpha_{i}, \alpha \beta_{i j}, \varepsilon_{i k}$ e $\epsilon_{i j k}$ & 120 & 12 & 10 \\
\hline
\end{tabular}

*DBC: Delineamento casualizado em blocos; PSD: Parcelas subdivididas

Em cada modelo trabalhado considerou-se os efeitos fixos dos fatores iguais a zero, essa consideração foi necessária para manter pequena a diferença entre as médias dos tratamentos e assim simular a hipótese nula $H_{0}: \alpha_{1}=\alpha_{2}=\cdots=\alpha_{a}=0$, pois quando a diferença entre as médias de tratamentos for grande o teste $F$ detecta esta diferença com poder igual a 1, o que impossibilita a observação de diferenças entre os valores do poder do teste. 
Nas simulações de cada uma das configurações dos modelos (13) e (14) foram comparados os quatro métodos apresentados na seção 2.4 utilizados no cálculo do número aproximado de graus de liberdade do denominador da estatística Wald- $F$. Em cada cenário estudado, foram simulados 1000 conjuntos de dados para obtenção do poder simulado do teste Wald- $F$ e taxa de erro tipo I, os quais foram utilizados como critérios de comparação entre os quatro métodos.

Para ilustrar o processo de simulação será utilizado, como exemplo, o Modelo I, considerando $\sigma_{B l}^{2}=0,1$ e $\sigma_{R}^{2}=1$, utilizando o método Containment para o cálculo do número aproximado de graus de liberdade para o teste $F$.

Foi gerado um conjunto com 30 observações fixando-se a semente, a variância residual e a variância do bloco. O desbalanceamento dos dados foi realizado utilizando-se o procedimento PROC PLAN do SAS para sortear as 3 observações perdidas com o seguinte código:

PROC PLAN;

FACTORS parc $=3$ of 30 ;

RUN;

O código para geração desse conjunto de dados com as observações perdidas é o que se segue:

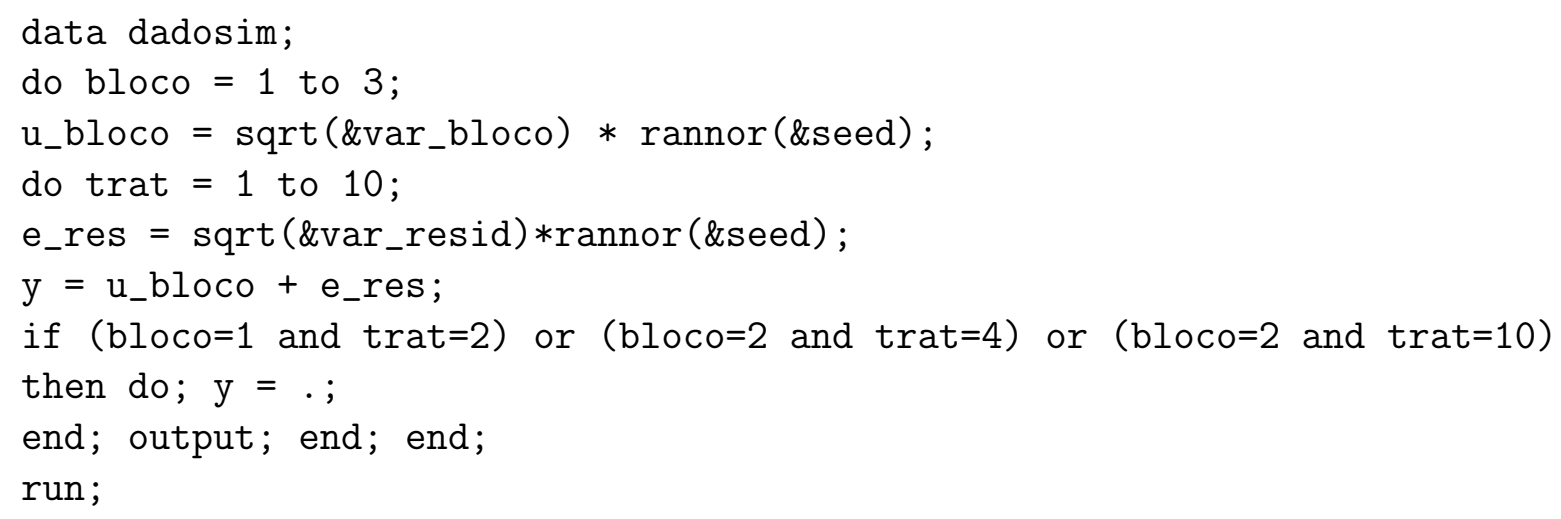

O conjunto de dados gerados foi utilizado para o cálculo do poder analítico do teste Wald- $F$, que foi realizado por meio do procedimento PROC MIXED do SAS. Nesse procedimento os fatores de efeitos fixos do modelo devem ser colocados à frente do comando MODEL e todos os fatores de efeitos aleatórios devem ser especificados à frente do comando RANDON. No comando DATA deve ser especificado o nome do conjunto de dados que será analisado. O método de estimação dos componentes de variância é especificado 
utilizando-se o comando method. Para modificar os métodos para o cálculo do número de graus de liberdade é utilizada a opção ddfm do comando MODEL, nesse exemplo ilustrativo considerou-se o método Containment. No comando ods output tests3=t1 é informado ao SAS que os resultados do teste $F$ serão armazenados no arquivo t1. O código para esse exemplo é o que se segue:

PROC MIXED Data=dadosim method=REML;

CLASS bloco trat ;

MODEL y = trat / ddfm=contain;

RANDON bloco;

ods output tests3=t 1 ;

RUN;

Para o cálculo do poder analítico utilizou-se como base as ideias de Helms (1992), Stroup (2002), Tempelman (2005), Rosa et al. (2005), que mostraram como calcular uma aproximação do poder de um teste $F$, utilizando para isso a estatística $F$ para calcular o parâmetro de não centralidade, necessário para o cálculo do poder do teste. O parâmetro de não centralidade é calculado por meio do produto do número de graus de liberdade do numerador da estatística $F$ e o próprio valor da estatística. O código utilizado para tal procedimento é o que se segue:

data f_power;

set $\mathrm{t} 1$;

Noncen $=$ NumDF $*$ FValue;

Alpha $=0.05$

FCrit = finv(1-Alpha, NumDF, DenDF, 0);

Power $=1-\operatorname{probf}($ FCrit, NumDF, DenDF, Noncen);

run;

Para o cálculo da taxa de erro tipo I e do poder simulado do teste utilizou-se como base as ideias de Littell et al. (2006), sendo gerados 1000 conjuntos de dados com 30 observações cada, o que é feito acrescentando-se as linhas de comandos

do $\operatorname{sim}=1$ to 1000 ;

end;

nas devidas posições do código apresentado anteriormente para a geração das 30 observações com os dados faltantes. Isso é necessário para a realização do looping gerador dos 1000 conjuntos de dados com os mesmos dados faltantes do conjunto inicial.

A taxa de erro tipo I foi calculada realizando o teste Wald- $F$ para cada um dos 1000 conjuntos de dados simulados considerando o modelo cuja variável resposta 
correspondente foi a soma dos efeitos aleatórios dos 1000 conjuntos de dados simulados. Uma vez que a taxa de erro tipo I é a probabilidade de rejeitar $H_{0}$ quando $H_{0}$ é verdadeira, contaram-se as vezes em que se rejeitou $H_{0}$ nos 1000 testes realizados. Dessa forma, a proporção de rejeição de $H_{0}$ nos 1000 testes correspondeu à taxa de erro tipo I.

O poder simulado foi calculado realizando o teste Wald- $F$ para cada um dos 1000 conjuntos de dados simulados. Para isso, considerou-se o modelo cuja variável resposta correspondente foi a soma dos valores preditos, do modelo inicial com o conjunto de 30 observações, e os efeitos aleatórios dos 1000 conjuntos de dados gerados anteriormente. Uma vez que o poder do teste é a probabilidade de rejeitar $H_{0}$ quando $H_{0}$ é falsa, contaram-se as vezes em que se rejeitou $H_{0}$ nos 1000 testes realizados. Assim, a proporção de rejeição de $H_{0}$ nos 1000 testes correspondeu ao poder simulado. 


\section{RESULTADOS E DISCUSSÃO}

De acordo com a metodologia e o processo de simulação abordados na seção (3) serão apresentados os resultados do poder analítico, do poder simulado e da taxa de erro tipo I, considerando os modelos apresentados na Tabela 2. Considerou-se nesse processo os cenários de análise em um delineamento casualizado em blocos com um fator de tratamento e um delineamento casualizado em blocos com tratamentos no esquema de parcelas subdivididas, com diferentes tamanhos amostrais, 30, 60 e 120 observações, diferentes níveis de desbalanceamento (10\%, 20\% e 30\%), diferentes valores para as variâncias do bloco e da parcela, fixando a variância residual em uma unidade, e quatro métodos de cálculo do número aproximado de graus de liberdade (Containment, Residual, Satterthwaite e Kenward-Roger).

\subsection{Delineamento casualizado em blocos (DBC)}

Nessa seção serão apresentados os resultados das simulações para os modelos (I, II e III) no delineamento casualizado em blocos com um fator de tratamento.

$D B C \operatorname{com} n=30$

Na Tabela 3 nota-se que o método Residual apresentou maior poder de teste para todas as situações, porém esse método apresentou maior taxa de erro tipo I em comparação aos demais métodos. Na maioria dos casos apresentados os métodos de Containment e Kenward-Roger controlaram melhor a taxa de erro tipo I do que os métodos de Satterthwaite e Residual, porém o poder de teste desses dois últimos métodos foi superior ao dos outros dois métodos.

Observa-se que o poder do teste foi maior quando a variância residual $\left(\sigma_{R}^{2}\right)$ foi superior à variância do bloco $\left(\sigma_{B l}^{2}\right)$ e que na maior parte dos casos, dentro de cada nível de desbalanceamento, o poder do teste foi maior quando a taxa de erro tipo I foi maior. Também nota-se que o poder simulado de teste distingue de modo mais promissor os métodos de cálculo do número aproximado de graus de liberdade do que o poder analítico. 
Tabela 3 - Poder analítico (P.A.), poder simulado (P.S.) e taxa de erro tipo I $(\hat{\alpha})$ para o Modelo I com níveis de desbalanceamento de 10\%, 20\% e 30\%

\begin{tabular}{|c|c|c|c|c|c|c|c|c|c|c|}
\hline \multirow{2}{*}{$\begin{array}{l}\text { Variação } \\
\left(\sigma_{B l}^{2} ; \sigma_{R}^{2}\right)\end{array}$} & \multirow{2}{*}{ Método* } & \multicolumn{3}{|c|}{$10 \%$} & \multicolumn{3}{|c|}{$20 \%$} & \multicolumn{3}{|c|}{$30 \%$} \\
\hline & & P.A. & P.S. & $\hat{\alpha}$ & P.A. & P.S. & $\hat{\alpha}$ & P.A. & P.S. & $\hat{\alpha}$ \\
\hline$(0,1 ; 1)$ & $\mathrm{C}$ & 0,210 & 0,195 & 0,053 & 0,219 & 0,227 & 0,049 & 0,163 & 0,176 & 0,045 \\
\hline$(0,1 ; 1)$ & $\mathrm{R}$ & 0,219 & 0,212 & 0,060 & 0,234 & 0,253 & 0,057 & 0,175 & 0,206 & 0,057 \\
\hline$(0,1 ; 1)$ & S & 0,219 & 0,203 & 0,055 & 0,234 & 0,241 & 0,054 & 0,175 & 0,183 & 0,048 \\
\hline$(0,1 ; 1)$ & $\mathrm{KR}$ & 0,219 & 0,197 & 0,054 & 0,234 & 0,237 & 0,051 & 0,175 & 0,174 & 0,044 \\
\hline$(0,5 ; 1)$ & $\mathrm{C}$ & 0,205 & 0,193 & 0,055 & 0,202 & 0,229 & 0,042 & 0,159 & 0,183 & 0,041 \\
\hline$(0,5 ; 1)$ & $\mathrm{R}$ & 0,214 & 0,220 & 0,057 & 0,215 & 0,253 & 0,056 & 0,170 & 0,225 & 0,052 \\
\hline$(0,5 ; 1)$ & S & 0,205 & 0,199 & 0,055 & 0,203 & 0,235 & 0,044 & 0,161 & 0,195 & 0,042 \\
\hline$(0,5 ; 1)$ & $\mathrm{KR}$ & 0,199 & 0,197 & 0,054 & 0,195 & 0,231 & 0,044 & 0,155 & 0,186 & 0,039 \\
\hline$(1 ; 1)$ & $\mathrm{C}$ & 0,198 & 0,185 & 0,054 & 0,192 & 0,218 & 0,042 & 0,153 & 0,176 & 0,040 \\
\hline$(1 ; 1)$ & $\mathrm{R}$ & 0,206 & 0,204 & 0,057 & 0,204 & 0,240 & 0,054 & 0,163 & 0,210 & 0,051 \\
\hline$(1 ; 1)$ & S & 0,198 & 0,187 & 0,055 & 0,192 & 0,226 & 0,042 & 0,153 & 0,183 & 0,041 \\
\hline$(1 ; 1)$ & $\mathrm{KR}$ & 0,196 & 0,184 & 0,055 & 0,190 & 0,220 & 0,042 & 0,151 & 0,178 & 0,039 \\
\hline$(5 ; 1)$ & $\mathrm{C}$ & 0,190 & 0,173 & 0,051 & 0,184 & 0,205 & 0,041 & 0,147 & 0,163 & 0,039 \\
\hline$(5 ; 1)$ & $\mathrm{R}$ & 0,198 & 0,185 & 0,056 & 0,195 & 0,229 & 0,052 & 0,157 & 0,202 & 0,051 \\
\hline$(5 ; 1)$ & S & 0,190 & 0,173 & 0,052 & 0,184 & 0,206 & 0,041 & 0,147 & 0,164 & 0,040 \\
\hline$(5 ; 1)$ & $\mathrm{KR}$ & 0,190 & 0,173 & 0,052 & 0,184 & 0,205 & 0,041 & 0,147 & 0,163 & 0,039 \\
\hline
\end{tabular}

${ }^{*}$ C:Containment, R:Residual, S: Satterthwaite e KR: Kenward-Roger.

$D B C \operatorname{com} n=60$

Na Tabela 4 observa-se que o método Residual apresentou maior poder de teste para todas as situações, porém esse método apresentou maior taxa de erro tipo I em comparação aos demais métodos. Na maior parte dos casos, os métodos de Containment e Kenward-Roger controlaram melhor a taxa de erro tipo I do que os métodos de Satterthwaite e Residual, porém o poder de teste desses dois últimos métodos foi superior aos outros dois.

Observa-se que o poder do teste foi superior quando a variância residual $\left(\sigma_{R}^{2}\right)$ foi maior em relação a variância do bloco $\left(\sigma_{B l}^{2}\right)$ e que na maioria dos casos, dentro de cada nível de desbalanceamento, o poder do teste foi maior quando a taxa de erro tipo I foi maior. Nota-se que os valores do poder do teste ficaram mais semelhantes entre si, bem como os valores da taxa de erro tipo I, do que os valores apresentados na Tabela 3, dentro de cada nível de desbalanceamento. Observa-se também que o poder de teste 
Tabela 4 - Poder analítico (P.A.), poder simulado (P.S.) e taxa de erro tipo I $(\hat{\alpha})$ para o Modelo II com níveis de desbalanceamento de 10\%, 20\% e $30 \%$

\begin{tabular}{|c|c|c|c|c|c|c|c|c|c|c|}
\hline \multirow{2}{*}{$\begin{array}{l}\text { Variação } \\
\left(\sigma_{B l}^{2} ; \sigma_{R}^{2}\right)\end{array}$} & \multirow{2}{*}{ Método* } & \multicolumn{3}{|c|}{$10 \%$} & \multicolumn{3}{|c|}{$20 \%$} & \multicolumn{3}{|c|}{$30 \%$} \\
\hline & & P.A. & P.S. & $\hat{\alpha}$ & P.A. & P.S. & $\hat{\alpha}$ & P.A. & P.S. & $\hat{\alpha}$ \\
\hline$(0,1 ; 1)$ & $\mathrm{C}$ & 0,132 & 0,144 & 0,057 & 0,190 & 0,165 & 0,053 & 0,224 & 0,219 & 0,066 \\
\hline$(0,1 ; 1)$ & $\mathrm{R}$ & 0,134 & 0,147 & 0,061 & 0,194 & 0,182 & 0,056 & 0,233 & 0,237 & 0,071 \\
\hline$(0,1 ; 1)$ & $\mathrm{S}$ & 0,134 & 0,146 & 0,059 & 0,194 & 0,175 & 0,053 & 0,233 & 0,227 & 0,068 \\
\hline$(0,1 ; 1)$ & $\mathrm{KR}$ & 0,134 & 0,144 & 0,057 & 0,194 & 0,170 & 0,052 & 0,233 & 0,216 & 0,065 \\
\hline$(0,5 ; 1)$ & $\mathrm{C}$ & 0,139 & 0,150 & 0,054 & 0,203 & 0,186 & 0,051 & 0,231 & 0,239 & 0,058 \\
\hline$(0,5 ; 1)$ & $\mathrm{R}$ & 0,141 & 0,156 & 0,057 & 0,209 & 0,192 & 0,056 & 0,240 & 0,256 & 0,064 \\
\hline$(0,5 ; 1)$ & $\mathrm{S}$ & 0,139 & 0,150 & 0,054 & 0,205 & 0,188 & 0,051 & 0,234 & 0,241 & 0,059 \\
\hline$(0,5 ; 1)$ & $\mathrm{KR}$ & 0,138 & 0,150 & 0,053 & 0,202 & 0,181 & 0,050 & 0,229 & 0,234 & 0,056 \\
\hline$(1 ; 1)$ & $\mathrm{C}$ & 0,137 & 0,145 & 0,054 & 0,206 & 0,188 & 0,053 & 0,222 & 0,229 & 0,056 \\
\hline$(1 ; 1)$ & $\mathrm{R}$ & 0,139 & 0,153 & 0,058 & 0,212 & 0,191 & 0,056 & 0,231 & 0,249 & 0,063 \\
\hline$(1 ; 1)$ & $\mathrm{S}$ & 0,137 & 0,147 & 0,054 & 0,207 & 0,188 & 0,054 & 0,224 & 0,233 & 0,058 \\
\hline$(1 ; 1)$ & $\mathrm{KR}$ & 0,136 & 0,144 & 0,053 & 0,206 & 0,186 & 0,052 & 0,222 & 0,225 & 0,056 \\
\hline$(5 ; 1)$ & $\mathrm{C}$ & 0,132 & 0,141 & 0,054 & 0,205 & 0,185 & 0,053 & 0,208 & 0,215 & 0,054 \\
\hline$(5 ; 1)$ & $\mathrm{R}$ & 0,134 & 0,145 & 0,057 & 0,210 & 0,196 & 0,057 & 0,216 & 0,231 & 0,059 \\
\hline$(5 ; 1)$ & $\mathrm{S}$ & 0,132 & 0,141 & 0,054 & 0,205 & 0,185 & 0,053 & 0,209 & 0,215 & 0,054 \\
\hline$(5 ; 1)$ & $\mathrm{KR}$ & 0,132 & 0,141 & 0,054 & 0,205 & 0,184 & 0,053 & 0,208 & 0,214 & 0,053 \\
\hline
\end{tabular}

${ }^{*}$ C:Containment, R:Residual, S: Satterthwaite e KR: Kenward-Roger.

simulado distingue de modo mais promissor os métodos de cálculo do número aproximado de graus de liberdade do que o poder analítico.

$D B C \operatorname{com} n=120$

Na Tabela 5 nota-se que o método Residual apresentou maior poder de teste para todas as situações, porém em alguns casos esse método apresentou maior taxa de erro tipo I em comparação aos demais métodos. Em alguns casos os métodos de Containment e Kenward-Roger controlaram melhor a taxa de erro tipo I do que os métodos de Satterthwaite e Residual, porém o poder de teste desses dois últimos métodos foi superior aos dois primeiros.

Observa-se que o poder do teste foi maior quando a variância residual $\left(\sigma_{R}^{2}\right)$ foi superior em relação a variância do bloco $\left(\sigma_{B l}^{2}\right)$ e que na maioria dos casos, dentro de cada nível de desbalanceamento, o poder do teste foi maior quando a taxa de erro tipo 
Tabela 5 - Poder analítico (P.A.), poder simulado (P.S.) e taxa de erro tipo I $(\hat{\alpha})$ para o Modelo III com níveis de desbalanceamento de 10\%, 20\% e 30\%

\begin{tabular}{|c|c|c|c|c|c|c|c|c|c|c|}
\hline \multirow{2}{*}{$\begin{array}{l}\text { Variação } \\
\left(\sigma_{B l}^{2} ; \sigma_{R}^{2}\right)\end{array}$} & \multirow{2}{*}{ Método* } & \multicolumn{3}{|c|}{$10 \%$} & \multicolumn{3}{|c|}{$20 \%$} & \multicolumn{3}{|c|}{$30 \%$} \\
\hline & & P.A. & P.S. & $\hat{\alpha}$ & P.A. & P.S. & $\hat{\alpha}$ & P.A. & P.S. & $\hat{\alpha}$ \\
\hline$(0,1 ; 1)$ & $\mathrm{C}$ & 0,750 & 0,764 & 0,039 & 0,803 & 0,804 & 0,039 & 0,520 & 0,439 & 0,035 \\
\hline$(0,1 ; 1)$ & $\mathrm{R}$ & 0,755 & 0,768 & 0,040 & 0,810 & 0,809 & 0,042 & 0,529 & 0,447 & 0,038 \\
\hline$(0,1 ; 1)$ & S & 0,750 & 0,765 & 0,039 & 0,805 & 0,807 & 0,041 & 0,524 & 0,443 & 0,036 \\
\hline$(0,1 ; 1)$ & $\mathrm{KR}$ & 0,749 & 0,762 & 0,039 & 0,803 & 0,803 & 0,039 & 0,520 & 0,436 & 0,034 \\
\hline$(0,5 ; 1)$ & $\mathrm{C}$ & 0,754 & 0,765 & 0,040 & 0,781 & 0,772 & 0,041 & 0,484 & 0,399 & 0,037 \\
\hline$(0,5 ; 1)$ & $\mathrm{R}$ & 0,759 & 0,772 & 0,043 & 0,788 & 0,782 & 0,043 & 0,493 & 0,409 & 0,039 \\
\hline$(0,5 ; 1)$ & S & 0,754 & 0,766 & 0,040 & 0,782 & 0,773 & 0,041 & 0,486 & 0,401 & 0,037 \\
\hline$(0,5 ; 1)$ & $\mathrm{KR}$ & 0,753 & 0,765 & 0,040 & 0,782 & 0,772 & 0,040 & 0,484 & 0,397 & 0,037 \\
\hline$(1 ; 1)$ & $\mathrm{C}$ & 0,754 & 0,765 & 0,042 & 0,775 & 0,765 & 0,041 & 0,478 & 0,393 & 0,040 \\
\hline$(1 ; 1)$ & $\mathrm{R}$ & 0,759 & 0,773 & 0,042 & 0,782 & 0,766 & 0,044 & 0,487 & 0,403 & 0,042 \\
\hline$(1 ; 1)$ & S & 0,754 & 0,765 & 0,042 & 0,775 & 0,842 & 0,047 & 0,479 & 0,394 & 0,040 \\
\hline$(1 ; 1)$ & $\mathrm{KR}$ & 0,754 & 0,763 & 0,042 & 0,775 & 0,764 & 0,041 & 0,478 & 0,393 & 0,040 \\
\hline$(5 ; 1)$ & $\mathrm{C}$ & 0,723 & 0,767 & 0,042 & 0,766 & 0,752 & 0,043 & 0,476 & 0,388 & 0,041 \\
\hline$(5 ; 1)$ & $\mathrm{R}$ & 0,758 & 0,772 & 0,044 & 0,773 & 0,757 & 0,043 & 0,485 & 0,395 & 0,042 \\
\hline$(5 ; 1)$ & S & 0,753 & 0,767 & 0,042 & 0,766 & 0,752 & 0,043 & 0,476 & 0,388 & 0,041 \\
\hline$(5 ; 1)$ & $\mathrm{KR}$ & 0,752 & 0,767 & 0,042 & 0,766 & 0,752 & 0,043 & 0,476 & 0,388 & 0,041 \\
\hline
\end{tabular}

${ }^{*}$ C:Containment, R:Residual, S: Satterthwaite e KR: Kenward-Roger.

I foi maior. Nota-se que os valores do poder do teste, bem como a taxa de erro tipo I, em muitos casos foram iguais entre si. Adicionalmente, observa-se que, dentro de cada nível de desbalanceamento, os valores do poder analítico ficaram mais semelhantes entre si, bem como o poder simulado e a taxa de erro tipo I do que os valores apresentados nas Tabelas 3 e 4 . Nota-se também, que para $n=120$ o poder do teste foi maior e o controle da taxa de erro tipo I foi melhor do que os casos de $n=30$ e $n=60$.

\subsection{Delineamento casualizado em blocos com tratamentos no esquema de parcela subdividida (DBC/PSD)}

Nessa seção serão apresentados os resultados das simulações para os modelos (IV, V, VI, VII, VIII e IX) no delineamento casualizado em blocos com tratamentos no esquema de parcelas subdivididas. 
$D B C / P S D \operatorname{com} n=30$ - interação $A B$

Na Tabela 6 nota-se que o método Residual apresentou maior poder de teste para todas as situações, porém esse método apresentou maior taxa de erro tipo I em comparação aos demais métodos. Na maioria dos casos apresentados, os métodos de Containment e Kenward-Roger controlaram melhor a taxa de erro tipo I do que os métodos de Satterthwaite e Residual, porém o poder de teste desses dois últimos métodos foi superior aos dois primeiros, sendo que para todos os casos o método de Kenward-Roger apresentou o poder do teste maior do que o método de Containment. Nota-se que em todos os casos o método de Satterthwaite apresentou o poder do teste maior do que o método de Kenward-Roger, porém esse último método controlou melhor a taxa de erro tipo I.

Tabela 6 - Poder analítico (P.A.), poder simulado (P.S.) e taxa de erro tipo I $(\hat{\alpha})$ para a interação $A B$ no Modelo IV com desbalanceamentos de 10\%, 20\% e $30 \%$

\begin{tabular}{|c|c|c|c|c|c|c|c|c|c|c|}
\hline \multirow{2}{*}{$\begin{array}{c}\text { Variação } \\
\left(\sigma_{B l}^{2}, \sigma_{P}^{2}, \sigma_{R}^{2}\right)\end{array}$} & \multirow{2}{*}{ Método* } & \multicolumn{3}{|c|}{$10 \%$} & \multicolumn{3}{|c|}{$20 \%$} & \multicolumn{3}{|c|}{$30 \%$} \\
\hline & & P.A. & P.S. & $\hat{\alpha}$ & P.A. & P.S. & $\hat{\alpha}$ & P.A. & P.S. & $\hat{\alpha}$ \\
\hline$(0,1 ; 5 ; 1)$ & $\mathrm{C}$ & 0,092 & 0,099 & 0,068 & 0,074 & 0,104 & 0,068 & 0,057 & 0,079 & 0,066 \\
\hline$(0,1 ; 5 ; 1)$ & $\mathrm{R}$ & 0,100 & 0,151 & 0,088 & 0,083 & 0,168 & 0,132 & 0,062 & 0,181 & 0,146 \\
\hline$(0,1 ; 5 ; 1)$ & S & 0,092 & 0,101 & 0,069 & 0,076 & 0,114 & 0,077 & 0,059 & 0,087 & 0,069 \\
\hline$(0,1 ; 5 ; 1)$ & $\mathrm{KR}$ & 0,092 & 0,099 & 0,067 & 0,075 & 0,107 & 0,073 & 0,058 & 0,080 & 0,066 \\
\hline$(0,5 ; 0,1 ; 1)$ & $\mathrm{C}$ & 0,095 & 0,101 & 0,067 & 0,103 & 0,102 & 0,067 & 0,055 & 0,064 & 0,065 \\
\hline$(0,5 ; 0,1 ; 1)$ & $\mathrm{R}$ & 0,104 & 0,151 & 0,096 & 0,124 & 0,180 & 0,136 & 0,058 & 0,161 & 0,149 \\
\hline$(0,5 ; 0,1 ; 1)$ & S & 0,103 & 0,122 & 0,074 & 0,121 & 0,134 & 0,102 & 0,059 & 0,104 & 0,095 \\
\hline$(0,5 ; 0,1 ; 1)$ & $\mathrm{KR}$ & 0,102 & 0,116 & 0,073 & 0,120 & 0,125 & 0,087 & 0,057 & 0,086 & 0,075 \\
\hline$(0,1 ; 0,5 ; 1)$ & $\mathrm{C}$ & 0,098 & 0,117 & 0,066 & 0,099 & 0,112 & 0,064 & 0,063 & 0,071 & 0,062 \\
\hline$(0,1 ; 0,5 ; 1)$ & $\mathrm{R}$ & 0,109 & 0,165 & 0,093 & 0,117 & 0,196 & 0,137 & 0,071 & 0,178 & 0,146 \\
\hline$(0,1 ; 0,5 ; 1)$ & S & 0,102 & 0,130 & 0,076 & 0,115 & 0,145 & 0,094 & 0,070 & 0,112 & 0,091 \\
\hline$(0,1 ; 0,5 ; 1)$ & $\mathrm{KR}$ & 0,099 & 0,126 & 0,072 & 0,114 & 0,133 & 0,074 & 0,067 & 0,097 & 0,076 \\
\hline$(1 ; 1 ; 1)$ & $\mathrm{C}$ & 0,098 & 0,112 & 0,070 & 0,081 & 0,107 & 0,065 & 0,056 & 0,070 & 0,065 \\
\hline$(1 ; 1 ; 1)$ & $\mathrm{R}$ & 0,108 & 0,159 & 0,094 & 0,093 & 0,178 & 0,134 & 0,060 & 0,158 & 0,145 \\
\hline$(1 ; 1 ; 1)$ & S & 0,101 & 0,123 & 0,072 & 0,087 & 0,132 & 0,084 & 0,059 & 0,090 & 0,080 \\
\hline$(1 ; 1 ; 1)$ & $\mathrm{KR}$ & 0,099 & 0,113 & 0,069 & 0,085 & 0,117 & 0,057 & 0,057 & 0,078 & 0,065 \\
\hline
\end{tabular}

${ }^{*}$ C:Containment, R:Residual, S: Satterthwaite e KR: Kenward-Roger.

Observa-se que o poder do teste foi maior quando a variância residual $\left(\sigma_{R}^{2}\right)$ foi superior a variância da parcela $\left(\sigma_{P}^{2}\right)$, e o poder simulado foi maior do que o poder 
analítico. Observa-se na maioria dos casos, dentro de cada nível de desbalanceamento, que a taxa de erro tipo I foi maior quando o poder do teste foi maior. Nota-se também que com o aumento na porcentagem de desbalanceamento o poder do teste diminui e que o poder de teste simulado distingue de modo mais promissor os métodos de cálculo do número aproximado de graus de liberdade do que o poder analítico.

$D B C / P S D$ com $n=30-$ fator $A$

Na Tabela 7 nota-se que o método Residual apresentou maior poder de teste para todas as situações, porém esse método apresentou maior taxa de erro tipo I em comparação aos demais métodos.

Na maioria dos casos, os métodos de Containment e Kenward-Roger controlaram melhor a taxa de erro tipo I do que os métodos de Satterthwaite e Residual, no entanto o poder de teste desses dois últimos métodos foi superior aos outros dois, sendo que para todos os casos o método de Kenward-Roger apresentou o poder do teste maior do que o método de Containment. Nota-se também que o método de Satterthwaite produziu um poder do teste maior do que o método de Kenward-Roger, porém esse último método controlou melhor a taxa de erro tipo I.

Observa-se que o poder do teste foi maior quando a variância da parcela, $\sigma_{P}^{2}$, foi menor do que a variância residual, $\sigma_{R}^{2}$, e que na maioria dos casos com o aumento na porcentagem de desbalanceamento o poder o teste diminui e houve um melhor controle da taxa de erro tipo I para o menor grau de desbalanceamento. Observa-se também, que a taxa de erro tipo I foi melhor controlada quando a variância associada às parcelas foi menor e que, dentro de cada nível de desbalanceamento, o poder do teste foi maior quando a taxa de erro tipo I foi maior.

Adicionalmente, observa-se que o poder simulado de teste distingue de modo mais promissor os métodos de cálculo do número de graus de liberdade aproximado do que o poder analítico. 
Tabela 7 - Poder analítico (P.A.), poder simulado (P.S.) e taxa de erro tipo I ( $\hat{\alpha})$ para o fator $A$ no Modelo IV com desbalanceamentos de $10 \%, 20 \%$ e $30 \%$

\begin{tabular}{|c|c|c|c|c|c|c|c|c|c|c|}
\hline \multirow{2}{*}{$\begin{array}{c}\text { Variação } \\
\left(\sigma_{B l}^{2}, \sigma_{P}^{2}, \sigma_{R}^{2}\right)\end{array}$} & \multirow{2}{*}{ Método* } & \multicolumn{3}{|c|}{$10 \%$} & \multicolumn{3}{|c|}{$20 \%$} & \multicolumn{3}{|c|}{$30 \%$} \\
\hline & & P.A. & P.S. & $\hat{\alpha}$ & P.A. & P.S. & $\hat{\alpha}$ & P.A. & P.S. & $\hat{\alpha}$ \\
\hline$(0,1 ; 5 ; 1)$ & $\mathrm{C}$ & 0,086 & 0,093 & 0,056 & 0,067 & 0,071 & 0,054 & 0,064 & 0,077 & 0,057 \\
\hline$(0,1 ; 5 ; 1)$ & $\mathrm{R}$ & 0,095 & 0,154 & 0,096 & 0,070 & 0,123 & 0,097 & 0,068 & 0,119 & 0,099 \\
\hline$(0,1 ; 5 ; 1)$ & S & 0,091 & 0,104 & 0,065 & 0,069 & 0,079 & 0,062 & 0,066 & 0,086 & 0,067 \\
\hline$(0,1 ; 5 ; 1)$ & $\mathrm{KR}$ & 0,091 & 0,104 & 0,063 & 0,069 & 0,077 & 0,061 & 0,066 & 0,077 & 0,057 \\
\hline$(0,5 ; 0,1 ; 1)$ & $\mathrm{C}$ & 0,277 & 0,147 & 0,022 & 0,111 & 0,061 & 0,033 & 0,094 & 0,052 & 0,033 \\
\hline$(0,5 ; 0,1 ; 1)$ & $\mathrm{R}$ & 0,340 & 0,236 & 0,052 & 0,125 & 0,103 & 0,058 & 0,105 & 0,104 & 0,056 \\
\hline$(0,5 ; 0,1 ; 1)$ & S & 0,331 & 0,189 & 0,037 & 0,122 & 0,072 & 0,041 & 0,102 & 0,079 & 0,044 \\
\hline$(0,5 ; 0,1 ; 1)$ & $\mathrm{KR}$ & 0,328 & 0,184 & 0,035 & 0,120 & 0,067 & 0,039 & 0,096 & 0,063 & 0,036 \\
\hline$(0,1 ; 0,5 ; 1)$ & $\mathrm{C}$ & 0,227 & 0,148 & 0,041 & 0,080 & 0,062 & 0,036 & 0,062 & 0,041 & 0,035 \\
\hline$(0,1 ; 0,5 ; 1)$ & $\mathrm{R}$ & 0,276 & 0,238 & 0,070 & 0,087 & 0,102 & 0,078 & 0,065 & 0,179 & 0,072 \\
\hline$(0,1 ; 0,5 ; 1)$ & S & 0,223 & 0,182 & 0,052 & 0,086 & 0,079 & 0,050 & 0,064 & 0,065 & 0,054 \\
\hline$(0,1 ; 0,5 ; 1)$ & $\mathrm{KR}$ & 0,216 & 0,178 & 0,050 & 0,084 & 0,073 & 0,046 & 0,063 & 0,057 & 0,045 \\
\hline$(1 ; 1 ; 1)$ & $\mathrm{C}$ & 0,162 & 0,146 & 0,042 & 0,080 & 0,073 & 0,045 & 0,060 & 0,049 & 0,043 \\
\hline$(1 ; 1 ; 1)$ & $\mathrm{R}$ & 0,193 & 0,222 & 0,084 & 0,086 & 0,126 & 0,074 & 0,063 & 0,082 & 0,072 \\
\hline$(1 ; 1 ; 1)$ & S & 0,162 & 0,158 & 0,054 & 0,080 & 0,091 & 0,049 & 0,060 & 0,062 & 0,049 \\
\hline$(1 ; 1 ; 1)$ & $\mathrm{KR}$ & 0,160 & 0,155 & 0,051 & 0,079 & 0,086 & 0,045 & 0,058 & 0,056 & 0,045 \\
\hline
\end{tabular}

${ }^{*} \mathrm{C}$ :Containment, R:Residual, S: Satterthwaite e KR: Kenward-Roger.

$D B C / P S D$ com $n=30-$ fator $B$

Na Tabela 8 observa-se que o método Residual apresentou maior poder de teste para todas as situações, porém esse método apresentou maior taxa de erro tipo I em comparação aos demais métodos.

Na maioria dos casos, os métodos de Containment e Kenward-Roger controlaram melhor a taxa de erro tipo I do que os métodos de Satterthwaite e Residual no entanto o poder de teste desses dois últimos métodos foi superior aos outros dois, sendo que para todos os casos o método de Kenward-Roger apresentou o poder do teste maior do que o método de Containment. Nota-se que em todos os casos da Tabela 8 o método de Satterthwaite apresentou o poder do teste maior do que o método de Kenward-Roger, porém esse último método controlou melhor a taxa de erro tipo I.

Observa-se, na maioria dos casos, dentro dos valores de cada variação e de cada nível de desbalanceamento, que o poder do teste é maior quando a taxa de erro tipo 
Tabela 8 - Poder analítico (P.A.), poder simulado (P.S.) e taxa de erro tipo I ( $\hat{\alpha})$ para o fator $B$ no Modelo IV com desbalanceamentos de $10 \%, 20 \%$ e $30 \%$

\begin{tabular}{|c|c|c|c|c|c|c|c|c|c|c|}
\hline \multirow{2}{*}{$\begin{array}{c}\text { Variação } \\
\left(\sigma_{B l}^{2}, \sigma_{P}^{2}, \sigma_{R}^{2}\right)\end{array}$} & \multirow{2}{*}{ Método* } & \multicolumn{3}{|c|}{$10 \%$} & \multicolumn{3}{|c|}{$20 \%$} & \multicolumn{3}{|c|}{$30 \%$} \\
\hline & & P.A. & P.S. & $\hat{\alpha}$ & P.A. & P.S. & $\hat{\alpha}$ & P.A. & P.S. & $\hat{\alpha}$ \\
\hline$(0,1 ; 5 ; 1)$ & $\mathrm{C}$ & 0,050 & 0,042 & 0,044 & 0,050 & 0,049 & 0,049 & 0,053 & 0,059 & 0,049 \\
\hline$(0,1 ; 5 ; 1)$ & $\mathrm{R}$ & 0,050 & 0,058 & 0,058 & 0,050 & 0,078 & 0,083 & 0,054 & 0,115 & 0,100 \\
\hline$(0,1 ; 5 ; 1)$ & S & 0,050 & 0,044 & 0,044 & 0,050 & 0,052 & 0,052 & 0,053 & 0,072 & 0,056 \\
\hline$(0,1 ; 5 ; 1)$ & $\mathrm{KR}$ & 0,050 & 0,040 & 0,043 & 0,050 & 0,048 & 0,050 & 0,053 & 0,064 & 0,050 \\
\hline$(0,5 ; 0,1 ; 1)$ & $\mathrm{C}$ & 0,051 & 0,050 & 0,049 & 0,058 & 0,055 & 0,054 & 0,052 & 0,052 & 0,056 \\
\hline$(0,5 ; 0,1 ; 1)$ & $\mathrm{R}$ & 0,051 & 0,067 & 0,067 & 0,060 & 0,081 & 0,088 & 0,053 & 0,106 & 0,095 \\
\hline$(0,5 ; 0,1 ; 1)$ & $\mathrm{S}$ & 0,051 & 0,057 & 0,059 & 0,060 & 0,069 & 0,076 & 0,053 & 0,075 & 0,073 \\
\hline$(0,5 ; 0,1 ; 1)$ & $\mathrm{KR}$ & 0,051 & 0,056 & 0,057 & 0,060 & 0,064 & 0,070 & 0,053 & 0,062 & 0,059 \\
\hline$(0,1 ; 0,5 ; 1)$ & $\mathrm{C}$ & 0,050 & 0,041 & 0,043 & 0,051 & 0,053 & 0,052 & 0,051 & 0,057 & 0,056 \\
\hline$(0,1 ; 0,5 ; 1)$ & $\mathrm{R}$ & 0,050 & 0,058 & 0,056 & 0,051 & 0,082 & 0,081 & 0,052 & 0,108 & 0,099 \\
\hline$(0,1 ; 0,5 ; 1)$ & $\mathrm{S}$ & 0,050 & 0,047 & 0,048 & 0,051 & 0,068 & 0,065 & 0,052 & 0,078 & 0,074 \\
\hline$(0,1 ; 0,5 ; 1)$ & $\mathrm{KR}$ & 0,050 & 0,045 & 0,046 & 0,051 & 0,061 & 0,060 & 0,052 & 0,070 & 0,061 \\
\hline$(1 ; 1 ; 1)$ & $\mathrm{C}$ & 0,050 & 0,044 & 0,045 & 0,051 & 0,049 & 0,055 & 0,056 & 0,067 & 0,055 \\
\hline$(1 ; 1 ; 1)$ & $\mathrm{R}$ & 0,050 & 0,057 & 0,054 & 0,051 & 0,085 & 0,084 & 0,059 & 0,123 & 0,095 \\
\hline$(1 ; 1 ; 1)$ & $\mathrm{S}$ & 0,050 & 0,048 & 0,046 & 0,051 & 0,063 & 0,064 & 0,058 & 0,084 & 0,067 \\
\hline$(1 ; 1 ; 1)$ & $\mathrm{KR}$ & 0,050 & 0,046 & 0,046 & 0,051 & 0,058 & 0,071 & 0,056 & 0,072 & 0,062 \\
\hline
\end{tabular}

${ }^{*}$ C:Containment, R:Residual, S: Satterthwaite e KR: Kenward-Roger.

I é maior e que os valores do poder analítico foram semelhantes entre si para todos os métodos. Nota-se também na maioria dos casos que o poder do teste foi maior quando a variância da parcela, $\sigma_{P}^{2}$, foi menor que a variância residual, $\sigma_{R}^{2}$, e que quando se aumentou a porcentagem de desbalanceamento o poder o teste aumentou, porém houve um melhor controle da taxa de erro tipo I para o menor grau de desbalanceamento.

$D B C / P S D$ com $n=30$ - fator $B$ fixo e fator $A$ aleatório

Na Tabela 9 nota-se que o método Residual apresentou maior poder simulado de teste para todas as situações, porém esse método apresentou maior taxa de erro tipo I em comparação aos demais métodos.

Para todos os casos apresentados, o método de Containment apresentou a taxa de erro tipo I severamente tendenciosa e o poder de teste simulado bem menor do que o poder simulado dos outros métodos, já o método de Kenward-Roger foi o que melhor 
controlou a taxa de erro tipo I. O método de Satterthwaite apresentou o poder analítico igual ao método de Kenward-Roger na maioria dos casos, já o poder simulado foi maior em todos os casos.

Tabela 9 - Poder analítico (P.A.), poder simulado (P.S.) e taxa de erro tipo I ( $\hat{\alpha})$ para o fator $B$ no Modelo VII, considerando $\sigma_{A}^{2}=\sigma_{A B}^{2}=0,1$, com desbalanceamentos de $10 \%$, $20 \%$ e $30 \%$

\begin{tabular}{|c|c|c|c|c|c|c|c|c|c|c|}
\hline \multirow{2}{*}{$\begin{array}{c}\text { Variação } \\
\left(\sigma_{B l}^{2}, \sigma_{P}^{2}, \sigma_{R}^{2}\right)\end{array}$} & \multirow{2}{*}{ Método* } & \multicolumn{3}{|c|}{$10 \%$} & \multicolumn{3}{|c|}{$20 \%$} & \multicolumn{3}{|c|}{$30 \%$} \\
\hline & & P.A. & P.S. & $\hat{\alpha}$ & P.A. & P.S. & $\hat{\alpha}$ & P.A. & P.S. & $\hat{\alpha}$ \\
\hline$(0,1 ; 5 ; 1)$ & $\mathrm{C}$ & 0,075 & 0,005 & 0,000 & 0,072 & 0,005 & 0,002 & 0,055 & 0,002 & 0,006 \\
\hline$(0,1 ; 5 ; 1)$ & $\mathrm{R}$ & 0,109 & 0,139 & 0,043 & 0,101 & 0,109 & 0,065 & 0,060 & 0,082 & 0,069 \\
\hline$(0,1 ; 5 ; 1)$ & S & 0,074 & 0,087 & 0,022 & 0,073 & 0,066 & 0,040 & 0,055 & 0,049 & 0,035 \\
\hline$(0,1 ; 5 ; 1)$ & $\mathrm{KR}$ & 0,074 & 0,084 & 0,022 & 0,072 & 0,061 & 0,039 & 0,055 & 0,043 & 0,029 \\
\hline$(0,5 ; 0,1 ; 1)$ & $\mathrm{C}$ & 0,078 & 0,004 & 0,000 & 0,079 & 0,005 & 0,002 & 0,057 & 0,001 & 0,006 \\
\hline$(0,5 ; 0,1 ; 1)$ & $\mathrm{R}$ & 0,117 & 0,138 & 0,055 & 0,119 & 0,139 & 0,072 & 0,066 & 0,101 & 0,067 \\
\hline$(0,5 ; 0,1 ; 1)$ & S & 0,077 & 0,105 & 0,043 & 0,079 & 0,094 & 0,047 & 0,059 & 0,064 & 0,049 \\
\hline$(0,5 ; 0,1 ; 1)$ & $\mathrm{KR}$ & 0,077 & 0,101 & 0,040 & 0,077 & 0,084 & 0,043 & 0,059 & 0,059 & 0,043 \\
\hline$(0,1 ; 0,5 ; 1)$ & $\mathrm{C}$ & 0,072 & 0,005 & 0,000 & 0,079 & 0,005 & 0,002 & 0,057 & 0,002 & 0,006 \\
\hline$(0,1 ; 0,5 ; 1)$ & $\mathrm{R}$ & 0,107 & 0,137 & 0,051 & 0,119 & 0,133 & 0,072 & 0,066 & 0,104 & 0,064 \\
\hline$(0,1 ; 0,5 ; 1)$ & S & 0,072 & 0,102 & 0,039 & 0,079 & 0,088 & 0,051 & 0,057 & 0,062 & 0,043 \\
\hline$(0,1 ; 0,5 ; 1)$ & $\mathrm{KR}$ & 0,072 & 0,100 & 0,036 & 0,078 & 0,078 & 0,044 & 0,057 & 0,051 & 0,040 \\
\hline$(1 ; 1 ; 1)$ & $\mathrm{C}$ & 0,070 & 0,005 & 0,000 & 0,075 & 0,007 & 0,002 & 0,056 & 0,002 & 0,006 \\
\hline$(1 ; 1 ; 1)$ & $\mathrm{R}$ & 0,098 & 0,133 & 0,047 & 0,110 & 0,130 & 0,072 & 0,064 & 0,106 & 0,066 \\
\hline$(1 ; 1 ; 1)$ & S & 0,070 & 0,092 & 0,033 & 0,076 & 0,086 & 0,045 & 0,056 & 0,057 & 0,038 \\
\hline$(1 ; 1 ; 1)$ & $\mathrm{KR}$ & 0,070 & 0,087 & 0,032 & 0,075 & 0,080 & 0,040 & 0,056 & 0,050 & 0,034 \\
\hline
\end{tabular}

${ }^{*}$ C:Containment, R:Residual, S: Satterthwaite e KR: Kenward-Roger.

Observa-se na maioria dos casos que o poder do teste foi maior quando a variância da parcela, $\sigma_{P}^{2}$, foi menor que a variância residual, $\sigma_{R}^{2}$, e que com o aumento na porcentagem de desbalanceamento, o poder do teste diminuiu e a taxa de erro tipo I aumentou, assim houve um melhor controle da taxa de erro tipo I para o menor grau de desbalanceamento. Nota-se também na maioria dos casos, dentro dos valores de cada variação e de cada nível de desbalanceamento, que o poder do teste foi maior quando a taxa de erro tipo I foi maior. Adicionalmente, observa-se que o poder de teste simulado distingue de modo mais promissor os métodos de cálculo do número de graus de liberdade aproximado do que o poder analítico. 
$D B C / P S D$ com $n=60$ - interação $A B$

Na Tabela 10 nota-se que o método Residual apresentou maior poder de teste para todas as situações, porém esse método apresentou maior taxa de erro tipo I em comparação aos demais métodos.

Tabela 10 - Poder analítico (P.A.), poder simulado (P.S.) e taxa de erro tipo I $(\hat{\alpha})$ para a interação $A B$ no Modelo V com desbalanceamentos de 10\%, 20\% e $30 \%$

\begin{tabular}{|c|c|c|c|c|c|c|c|c|c|c|}
\hline \multirow{2}{*}{$\begin{array}{c}\text { Variação } \\
\left(\sigma_{B l}^{2}, \sigma_{P}^{2}, \sigma_{R}^{2}\right)\end{array}$} & \multirow{2}{*}{ Método* } & \multicolumn{3}{|c|}{$10 \%$} & \multicolumn{3}{|c|}{$20 \%$} & \multicolumn{3}{|c|}{$30 \%$} \\
\hline & & P.A. & P.S. & $\hat{\alpha}$ & P.A. & P.S. & $\hat{\alpha}$ & P.A. & P.S. & $\hat{\alpha}$ \\
\hline$(0,1 ; 5 ; 1)$ & $\mathrm{C}$ & 0,378 & 0,349 & 0,037 & 0,512 & 0,431 & 0,050 & 0,525 & 0,468 & 0,046 \\
\hline$(0,1 ; 5 ; 1)$ & $\mathrm{R}$ & 0,399 & 0,373 & 0,047 & 0,550 & 0,480 & 0,060 & 0,586 & 0,533 & 0,061 \\
\hline$(0,1 ; 5 ; 1)$ & $\mathrm{S}$ & 0,378 & 0,349 & 0,039 & 0,516 & 0,435 & 0,050 & 0,531 & 0,479 & 0,049 \\
\hline$(0,1 ; 5 ; 1)$ & $\mathrm{KR}$ & 0,378 & 0,349 & 0,038 & 0,513 & 0,430 & 0,048 & 0,516 & 0,459 & 0,044 \\
\hline$(0,5 ; 0,1 ; 1)$ & $\mathrm{C}$ & 0,363 & 0,358 & 0,040 & 0,476 & 0,425 & 0,045 & 0,413 & 0,442 & 0,042 \\
\hline$(0,5 ; 0,1 ; 1)$ & $\mathrm{R}$ & 0,384 & 0,384 & 0,046 & 0,512 & 0,473 & 0,056 & 0,465 & 0,513 & 0,057 \\
\hline$(0,5 ; 0,1 ; 1)$ & $\mathrm{S}$ & 0,363 & 0,367 & 0,044 & 0,480 & 0,446 & 0,052 & 0,414 & 0,482 & 0,049 \\
\hline$(0,5 ; 0,1 ; 1)$ & $\mathrm{KR}$ & 0,361 & 0,365 & 0,041 & 0,467 & 0,430 & 0,045 & 0,364 & 0,455 & 0,041 \\
\hline$(0,1 ; 0,5 ; 1)$ & $\mathrm{C}$ & 0,346 & 0,329 & 0,038 & 0,445 & 0,381 & 0,047 & 0,321 & 0,343 & 0,045 \\
\hline$(0,1 ; 0,5 ; 1)$ & $\mathrm{R}$ & 0,365 & 0,355 & 0,046 & 0,480 & 0,424 & 0,061 & 0,362 & 0,411 & 0,060 \\
\hline$(0,1 ; 0,5 ; 1)$ & $\mathrm{S}$ & 0,344 & 0,334 & 0,043 & 0,449 & 0,398 & 0,049 & 0,312 & 0,371 & 0,048 \\
\hline$(0,1 ; 0,5 ; 1)$ & $\mathrm{KR}$ & 0,343 & 0,330 & 0,039 & 0,442 & 0,383 & 0,048 & 0,291 & 0,339 & 0,043 \\
\hline$(1 ; 1 ; 1)$ & $\mathrm{C}$ & 0,371 & 0,341 & 0,039 & 0,517 & 0,449 & 0,047 & 0,532 & 0,502 & 0,047 \\
\hline$(1 ; 1 ; 1)$ & $\mathrm{R}$ & 0,392 & 0,372 & 0,045 & 0,556 & 0,497 & 0,057 & 0,593 & 0,578 & 0,066 \\
\hline$(1 ; 1 ; 1)$ & $\mathrm{S}$ & 0,371 & 0,342 & 0,041 & 0,522 & 0,457 & 0,050 & 0,540 & 0,522 & 0,050 \\
\hline$(1 ; 1 ; 1)$ & $\mathrm{KR}$ & 0,370 & 0,341 & 0,040 & 0,513 & 0,441 & 0,045 & 0,503 & 0,481 & 0,045 \\
\hline
\end{tabular}

${ }^{*}$ C:Containment, R:Residual, S: Satterthwaite e KR: Kenward-Roger.

Na maioria dos casos, os métodos de Containment e Kenward-Roger controlaram melhor a taxa de erro tipo I do que os métodos de Satterthwaite e Residual, no entanto o poder de teste desses dois últimos métodos foi superior aos outros dois, sendo que para todos os casos o método de Kenward-Roger apresentou o poder do teste maior do que o método de Containment. Nota-se também que em todos os casos apresentados o método de Satterthwaite produziu um poder do teste maior do que o método de Kenward-Roger, porém esse último método controlou melhor a taxa de erro tipo I.

Observa-se, na maioria dos casos apresentados na Tabela 10, que o poder do teste foi maior quando a taxa de erro tipo I foi maior, dentro de cada nível de desba- 
lanceamento. Nota-se também, que com o aumento na porcentagem de desbalanceamento o poder do teste aumentou, porém houve um melhor controle da taxa de erro tipo I para o menor grau de desbalanceamento.

Observa-se que, dentro de cada nível de desbalanceamento, os valores do poder analítico ficaram mais semelhantes entre si, bem como o poder simulado e a taxa de erro tipo I, do que os valores apresentados na Tabela 6 para $n=30$. No presente cenário o poder do teste foi maior e o controle da taxa de erro tipo I foi melhor do que a situação apresentada na Tabela 6 .

$D B C / P S D \operatorname{com} n=60-$ fator $A$

Na Tabela 11 nota-se que o método Residual apresentou maior poder de teste para todas as situações, porém esse método apresentou maior taxa de erro tipo I em comparação aos demais métodos.

Tabela 11 - Poder analítico (P.A.), poder simulado (P.S.) e taxa de erro tipo I $(\hat{\alpha})$ para o fator $A$ no Modelo $\mathrm{V}$ com desbalanceamentos de $10 \%$, 20\% e $30 \%$

\begin{tabular}{|c|c|c|c|c|c|c|c|c|c|c|}
\hline \multirow{2}{*}{$\begin{array}{c}\text { Variação } \\
\left(\sigma_{B l}^{2}, \sigma_{P}^{2}, \sigma_{R}^{2}\right)\end{array}$} & \multirow{2}{*}{ Método* } & \multicolumn{3}{|c|}{$10 \%$} & \multicolumn{3}{|c|}{$20 \%$} & \multicolumn{3}{|c|}{$30 \%$} \\
\hline & & P.A. & P.S. & $\hat{\alpha}$ & P.A. & P.S. & $\hat{\alpha}$ & P.A. & P.S. & $\hat{\alpha}$ \\
\hline$(0,1 ; 5 ; 1)$ & $\mathrm{C}$ & 0,399 & 0,221 & 0,062 & 0,382 & 0,221 & 0,072 & 0,384 & 0,248 & 0,068 \\
\hline$(0,1 ; 5 ; 1)$ & $\mathrm{R}$ & 0,520 & 0,351 & 0,106 & 0,493 & 0,336 & 0,113 & 0,489 & 0,364 & 0,108 \\
\hline$(0,1 ; 5 ; 1)$ & S & 0,444 & 0,242 & 0,073 & 0,429 & 0,251 & 0,081 & 0,424 & 0,280 & 0,076 \\
\hline$(0,1 ; 5 ; 1)$ & $\mathrm{KR}$ & 0,444 & 0,242 & 0,073 & 0,428 & 0,251 & 0,079 & 0,424 & 0,278 & 0,074 \\
\hline$(0,5 ; 0,1 ; 1)$ & $\mathrm{C}$ & 0,067 & 0,032 & 0,020 & 0,051 & 0,027 & 0,023 & 0,060 & 0,036 & 0,028 \\
\hline$(0,5 ; 0,1 ; 1)$ & $\mathrm{R}$ & 0,072 & 0,066 & 0,053 & 0,052 & 0,060 & 0,053 & 0,063 & 0,063 & 0,054 \\
\hline$(0,5 ; 0,1 ; 1)$ & S & 0,066 & 0,046 & 0,031 & 0,051 & 0,048 & 0,039 & 0,058 & 0,051 & 0,041 \\
\hline$(0,5 ; 0,1 ; 1)$ & $\mathrm{KR}$ & 0,066 & 0,046 & 0,031 & 0,051 & 0,044 & 0,036 & 0,057 & 0,047 & 0,039 \\
\hline$(0,1 ; 0,5 ; 1)$ & $\mathrm{C}$ & 0,115 & 0,103 & 0,040 & 0,094 & 0,084 & 0,050 & 0,135 & 0,102 & 0,051 \\
\hline$(0,1 ; 0,5 ; 1)$ & $\mathrm{R}$ & 0,139 & 0,175 & 0,088 & 0,109 & 0,147 & 0,096 & 0,162 & 0,176 & 0,092 \\
\hline$(0,1 ; 0,5 ; 1)$ & S & 0,120 & 0,121 & 0,052 & 0,098 & 0,110 & 0,064 & 0,126 & 0,129 & 0,066 \\
\hline$(0,1 ; 0,5 ; 1)$ & $\mathrm{KR}$ & 0,120 & 0,118 & 0,052 & 0,098 & 0,106 & 0,061 & 0,122 & 0,123 & 0,064 \\
\hline$(1 ; 1 ; 1)$ & $\mathrm{C}$ & 0,168 & 0,138 & 0,050 & 0,122 & 0,127 & 0,051 & 0,144 & 0,151 & 0,056 \\
\hline$(1 ; 1 ; 1)$ & $\mathrm{R}$ & 0,212 & 0,205 & 0,087 & 0,147 & 0,194 & 0,100 & 0,175 & 0,233 & 0,090 \\
\hline$(1 ; 1 ; 1)$ & S & 0,166 & 0,142 & 0,051 & 0,122 & 0,131 & 0,057 & 0,138 & 0,160 & 0,057 \\
\hline$(1 ; 1 ; 1)$ & $\mathrm{KR}$ & 0,170 & 0,141 & 0,051 & 0,121 & 0,125 & 0,055 & 0,137 & 0,157 & 0,054 \\
\hline
\end{tabular}

${ }^{*}$ C:Containment, R:Residual, S: Satterthwaite e KR: Kenward-Roger. 
Na maioria dos casos, os métodos de Containment e Kenward-Roger controlaram melhor a taxa de erro tipo I do que os métodos de Satterthwaite e Residual, no entanto o poder de teste desses dois últimos métodos foi superior aos outros dois, sendo que para todos os casos o método de Kenward-Roger apresentou o poder do teste maior do que o método de Containment. Nota-se também que em todos os casos o método de Satterthwaite produziu um poder do teste maior do que o método de Kenward-Roger, porém esse último método controlou melhor a taxa de erro tipo I.

Observa-se que o poder do teste foi maior quando a variância da parcela, $\sigma_{P}^{2}$, foi maior que as variâncias do bloco, $\sigma_{B l}^{2}$, e do resíduo, $\sigma_{R}^{2}$, porém houve um melhor controle da taxa de erro tipo I para o menor valor da variância da parcela.

Observa-se que, dentro dos valores de cada variação e de cada nível de desbalanceamento, o poder do teste foi maior quando a taxa de erro tipo I foi maior, e que houve um melhor controle da taxa de erro tipo I no menor grau de desbalanceamento e o poder do teste foi superior no maior grau de desbalanceamento. Nota-se também que o poder de teste simulado distingue de modo mais promissor os métodos de cálculo do número aproximado de graus de liberdade do que o poder analítico.

$D B C / P S D$ com $n=60-$ fator $B$

Na Tabela 12 nota-se que o método Residual apresentou maior poder de teste para todas as situações, porém esse método apresentou maior taxa de erro tipo I em comparação aos demais métodos.

Na maioria dos casos, os métodos de Containment e Kenward-Roger controlaram melhor a taxa de erro tipo I do que os métodos de Satterthwaite e Residual, no entanto o poder de teste desses dois últimos métodos foi superior aos outros dois, sendo que para todos os casos o método de Kenward-Roger apresentou o poder do teste maior do que o método de Containment. Nota-se também que em todos os casos o método de Satterthwaite produziu um poder do teste maior do que o método de Kenward-Roger, porém esse último método controlou melhor a taxa de erro tipo I.

Observa-se que o poder do teste foi superior quando a variância da parcela, $\sigma_{P}^{2}$, foi maior que as variâncias do bloco, $\sigma_{B l}^{2}$, e do resíduo, $\sigma_{R}^{2}$, porém houve um melhor controle da taxa de erro tipo I para o menor valor de variância da parcela.

Observa-se na maioria dos casos, dentro dos valores de cada variação e de cada nível de desbalanceamento, que o poder do teste foi maior quando a taxa de erro 
Tabela 12 - Poder analítico (P.A.), poder simulado (P.S.) e taxa de erro tipo I $(\hat{\alpha})$ para o fator $B$ no Modelo V com desbalanceamentos de $10 \%, 20 \%$ e $30 \%$

\begin{tabular}{|c|c|c|c|c|c|c|c|c|c|c|}
\hline \multirow{2}{*}{$\begin{array}{c}\text { Variação } \\
\left(\sigma_{B l}^{2}, \sigma_{P}^{2}, \sigma_{R}^{2}\right)\end{array}$} & \multirow{2}{*}{ Método* } & \multicolumn{3}{|c|}{$10 \%$} & \multicolumn{3}{|c|}{$20 \%$} & \multicolumn{3}{|c|}{$30 \%$} \\
\hline & & P.A. & P.S. & $\hat{\alpha}$ & P.A. & P.S. & $\hat{\alpha}$ & P.A. & P.S. & $\hat{\alpha}$ \\
\hline$(0,1 ; 5 ; 1)$ & $\mathrm{C}$ & 0,175 & 0,177 & 0,046 & 0,265 & 0,224 & 0,056 & 0,175 & 0,165 & 0,061 \\
\hline$(0,1 ; 5 ; 1)$ & $\mathrm{R}$ & 0,180 & 0,188 & 0,052 & 0,278 & 0,252 & 0,069 & 0,187 & 0,192 & 0,077 \\
\hline$(0,1 ; 5 ; 1)$ & S & 0,175 & 0,178 & 0,046 & 0,267 & 0,227 & 0,056 & 0,176 & 0,169 & 0,062 \\
\hline$(0,1 ; 5 ; 1)$ & $\mathrm{KR}$ & 0,175 & 0,177 & 0,046 & 0,265 & 0,223 & 0,056 & 0,171 & 0,161 & 0,061 \\
\hline$(0,5 ; 0,1 ; 1)$ & $\mathrm{C}$ & 0,165 & 0,177 & 0,049 & 0,248 & 0,243 & 0,063 & 0,109 & 0,124 & 0,062 \\
\hline$(0,5 ; 0,1 ; 1)$ & $\mathrm{R}$ & 0,169 & 0,187 & 0,054 & 0,260 & 0,267 & 0,069 & 0,115 & 0,149 & 0,076 \\
\hline$(0,5 ; 0,1 ; 1)$ & S & 0,165 & 0,179 & 0,049 & 0,250 & 0,253 & 0,065 & 0,110 & 0,140 & 0,072 \\
\hline$(0,5 ; 0,1 ; 1)$ & $\mathrm{KR}$ & 0,163 & 0,176 & 0,049 & 0,244 & 0,245 & 0,063 & 0,101 & 0,122 & 0,062 \\
\hline$(0,1 ; 0,5 ; 1)$ & $\mathrm{C}$ & 0,160 & 0,166 & 0,048 & 0,248 & 0,234 & 0,060 & 0,098 & 0,112 & 0,063 \\
\hline$(0,1 ; 0,5 ; 1)$ & $\mathrm{R}$ & 0,164 & 0,178 & 0,052 & 0,260 & 0,253 & 0,068 & 0,102 & 0,135 & 0,076 \\
\hline$(0,1 ; 0,5 ; 1)$ & S & 0,159 & 0,168 & 0,049 & 0,250 & 0,240 & 0,063 & 0,098 & 0,124 & 0,069 \\
\hline$(0,1 ; 0,5 ; 1)$ & $\mathrm{KR}$ & 0,158 & 0,165 & 0,049 & 0,246 & 0,234 & 0,060 & 0,092 & 0,108 & 0,062 \\
\hline$(1 ; 1 ; 1)$ & $\mathrm{C}$ & 0,171 & 0,174 & 0,047 & 0,262 & 0,234 & 0,061 & 0,153 & 0,157 & 0,060 \\
\hline$(1 ; 1 ; 1)$ & $\mathrm{R}$ & 0,176 & 0,185 & 0,052 & 0,275 & 0,259 & 0,071 & 0,163 & 0,178 & 0,074 \\
\hline$(1 ; 1 ; 1)$ & S & 0,171 & 0,174 & 0,047 & 0,265 & 0,239 & 0,064 & 0,155 & 0,160 & 0,067 \\
\hline$(1 ; 1 ; 1)$ & $\mathrm{KR}$ & 0,169 & 0,173 & 0,047 & 0,260 & 0,232 & 0,059 & 0,145 & 0,152 & 0,060 \\
\hline
\end{tabular}

${ }^{*} \mathrm{C}$ :Containment, R:Residual, S: Satterthwaite e KR: Kenward-Roger.

tipo I foi maior e os valores do poder analítico foram próximos entre si para os métodos de Containment, Satterthwaite e Kenward-Roger. Nota-se que houve um melhor controle da taxa de erro tipo I para o menor grau de desbalanceamento e que o poder de teste simulado distingue de modo mais promissor os métodos de cálculo do número aproximado de graus de liberdade do que o poder analítico.

Nota-se que os valores do poder do teste ficaram mais semelhantes entre si, bem como os valores da taxa de erro tipo I, do que os valores apresentados na Tabela 8 para $n=30$, dentro de cada nível de desbalanceamento. Observa-se também que o poder de teste simulado distingue de modo mais promissor os métodos de cálculo do número aproximado de graus de liberdade do que o poder analítico. No presente cenário o poder do teste foi maior e o controle da taxa de erro tipo I foi melhor do que a situação apresentada na Tabela 8. 
$D B C / P S D$ com $n=60$ - fator $B$ fixo e fator $A$ aleatório

Na Tabela 13 nota-se que o método Residual apresentou maior poder de teste para todas as situações, porém esse método apresentou maior taxa de erro tipo I em comparação aos demais métodos.

Tabela 13 - Poder analítico (P.A.), poder simulado (P.S.) e taxa de erro tipo I ( $\hat{\alpha})$ para o fator $B$ no Modelo VIII, considerando $\sigma_{A}^{2}=\sigma_{A B}^{2}=0,1$, com desbalanceamentos de $10 \%, 20 \%$ e $30 \%$

\begin{tabular}{|c|c|c|c|c|c|c|c|c|c|c|}
\hline \multirow{2}{*}{$\begin{array}{c}\text { Variação } \\
\left(\sigma_{B l}^{2}, \sigma_{P}^{2}, \sigma_{R}^{2}\right)\end{array}$} & \multirow{2}{*}{ Método* } & \multicolumn{3}{|c|}{$10 \%$} & \multicolumn{3}{|c|}{$20 \%$} & \multicolumn{3}{|c|}{$30 \%$} \\
\hline & & P.A. & P.S. & $\hat{\alpha}$ & P.A. & P.S. & $\hat{\alpha}$ & P.A. & P.S. & $\hat{\alpha}$ \\
\hline$(0,1 ; 5 ; 1)$ & $\mathrm{C}$ & 0,213 & 0,090 & 0,004 & 0,213 & 0,108 & 0,007 & 0,199 & 0,124 & 0,006 \\
\hline$(0,1 ; 5 ; 1)$ & $\mathrm{R}$ & 0,341 & 0,277 & 0,057 & 0,338 & 0,315 & 0,054 & 0,309 & 0,346 & 0,060 \\
\hline$(0,1 ; 5 ; 1)$ & S & 0,210 & 0,205 & 0,037 & 0,191 & 0,220 & 0,037 & 0,190 & 0,253 & 0,040 \\
\hline$(0,1 ; 5 ; 1)$ & $\mathrm{KR}$ & 0,211 & 0,204 & 0,037 & 0,187 & 0,216 & 0,036 & 0,185 & 0,248 & 0,039 \\
\hline$(0,5 ; 0,1 ; 1)$ & $\mathrm{C}$ & 0,224 & 0,094 & 0,004 & 0,198 & 0,121 & 0,008 & 0,175 & 0,113 & 0,007 \\
\hline$(0,5 ; 0,1 ; 1)$ & $\mathrm{R}$ & 0,360 & 0,306 & 0,054 & 0,311 & 0,347 & 0,056 & 0,267 & 0,346 & 0,059 \\
\hline$(0,5 ; 0,1 ; 1)$ & S & 0,226 & 0,243 & 0,043 & 0,180 & 0,275 & 0,041 & 0,176 & 0,273 & 0,045 \\
\hline$(0,5 ; 0,1 ; 1)$ & $\mathrm{KR}$ & 0,225 & 0,238 & 0,042 & 0,177 & 0,263 & 0,040 & 0,171 & 0,252 & 0,038 \\
\hline$(0,1 ; 0,5 ; 1)$ & $\mathrm{C}$ & 0,217 & 0,092 & 0,005 & 0,208 & 0,128 & 0,007 & 0,193 & 0,140 & 0,008 \\
\hline$(0,1 ; 0,5 ; 1)$ & $\mathrm{R}$ & 0,347 & 0,293 & 0,057 & 0,328 & 0,349 & 0,059 & 0,298 & 0,371 & 0,063 \\
\hline$(0,1 ; 0,5 ; 1)$ & S & 0,217 & 0,230 & 0,043 & 0,187 & 0,265 & 0,044 & 0,197 & 0,290 & 0,041 \\
\hline$(0,1 ; 0,5 ; 1)$ & $\mathrm{KR}$ & 0,216 & 0,223 & 0,040 & 0,183 & 0,257 & 0,042 & 0,187 & 0,280 & 0,039 \\
\hline$(1 ; 1 ; 1)$ & $\mathrm{C}$ & 0,220 & 0,094 & 0,004 & 0,195 & 0,114 & 0,007 & 0,173 & 0,122 & 0,004 \\
\hline$(1 ; 1 ; 1)$ & $\mathrm{R}$ & 0,353 & 0,294 & 0,057 & 0,305 & 0,330 & 0,053 & 0,262 & 0,342 & 0,062 \\
\hline$(1 ; 1 ; 1)$ & $\mathrm{S}$ & 0,221 & 0,229 & 0,040 & 0,173 & 0,246 & 0,039 & 0,166 & 0,254 & 0,041 \\
\hline$(1 ; 1 ; 1)$ & $\mathrm{KR}$ & 0,220 & 0,225 & 0,040 & 0,171 & 0,239 & 0,039 & 0,162 & 0,244 & 0,040 \\
\hline
\end{tabular}

${ }^{*}$ C:Containment, R:Residual, S: Satterthwaite e KR: Kenward-Roger.

Para todos os casos o método de Containment apresentou a taxa de erro tipo I severamente tendenciosa e o poder de teste simulado bem menor do que o poder simulado dos outros métodos, já o método de Kenward-Roger foi o que melhor controlou a taxa de erro tipo I. O método de Satterthwaite apresentou o poder simulado maior em todos os casos.

Observa-se na maioria dos casos que o poder do teste foi maior quando a variância da parcela, $\sigma_{P}^{2}$, foi menor que a variância residual, $\sigma_{R}^{2}$. 
Observa-se na maioria dos casos, dentro dos valores de cada variação e de cada nível de desbalanceamento, que o poder do teste foi maior quando a taxa de erro tipo I foi maior. Também nota-se que na maioria dos casos com o aumento na porcentagem de desbalanceamento o poder o teste diminuiu e a taxa de erro tipo I aumentou, assim houve um melhor controle da taxa de erro tipo I para o menor grau de desbalanceamento.

Nota-se também que os valores do poder do teste ficaram mais semelhantes entre si, bem como os valores da taxa de erro tipo I, do que os valores apresentados na Tabela 8 para $n=30$, dentro de cada nível de desbalanceamento. Observa-se também que o poder de teste simulado distingue de modo mais promissor os métodos de cálculo do número aproximado de graus de liberdade do que o poder analítico. No presente cenário o poder do teste foi maior e o controle da taxa de erro tipo I foi melhor do que a situação apresentada na Tabela 8 .

Observa-se também, dentro de cada nível de desbalanceamento, que os valores do poder do teste ficaram mais semelhantes entre si, bem como os valores da taxa de erro tipo I, do que os valores apresentados na Tabela 9 para $n=30$. No presente cenário o poder do teste foi maior e o controle da taxa de erro tipo I foi melhor do que a situação apresentada na Tabela 9 .

$D B C / P S D \operatorname{com} n=120$ - interação $A B$

Na Tabela 14 nota-se que o método Residual apresentou maior poder de teste para todas as situações, porém esse método apresentou maior taxa de erro tipo I em comparação aos demais métodos.

Na maioria dos casos, os métodos de Containment e Kenward-Roger controlaram melhor a taxa de erro tipo I do que os métodos de Satterthwaite e Residual, no entanto o poder de teste desses dois últimos métodos foi superior aos outros dois, sendo que para todos os casos o método de Kenward-Roger apresentou o poder do teste maior do que o método de Containment. Nota-se também que em todos os casos o método de Satterthwaite produziu um poder do teste maior do que o método de Kenward-Roger, porém esse último método controlou melhor a taxa de erro tipo I.

Observa-se que o poder do teste foi superior quando a variância da parcela, $\sigma_{P}^{2}$, foi menor que a variância residual, $\sigma_{R}^{2}$, e que para todos os casos o poder simulado foi maior do que o poder analítico. 
Tabela 14 - Poder analítico (P.A.), poder simulado (P.S.) e taxa de erro tipo I ( $\hat{\alpha})$ para a interação $A B$ no Modelo VI com desbalanceamentos de 10\%, 20\% e $30 \%$

\begin{tabular}{|c|c|c|c|c|c|c|c|c|c|c|}
\hline \multirow{2}{*}{$\begin{array}{c}\text { Variação } \\
\left(\sigma_{B l}^{2}, \sigma_{P}^{2}, \sigma_{R}^{2}\right)\end{array}$} & \multirow{2}{*}{ Método* } & \multicolumn{3}{|c|}{$10 \%$} & \multicolumn{3}{|c|}{$20 \%$} & \multicolumn{3}{|c|}{$30 \%$} \\
\hline & & P.A. & P.S. & $\hat{\alpha}$ & P.A. & P.S. & $\hat{\alpha}$ & P.A. & P.S. & $\hat{\alpha}$ \\
\hline$(0,1 ; 5 ; 1)$ & $\mathrm{C}$ & 0,893 & 0,866 & 0,049 & 0,762 & 0,668 & 0,050 & 0,619 & 0,551 & 0,058 \\
\hline$(0,1 ; 5 ; 1)$ & $\mathrm{R}$ & 0,911 & 0,879 & 0,064 & 0,801 & 0,701 & 0,064 & 0,677 & 0,626 & 0,078 \\
\hline$(0,1 ; 5 ; 1)$ & $\mathrm{S}$ & 0,894 & 0,867 & 0,049 & 0,766 & 0,672 & 0,053 & 0,630 & 0,569 & 0,062 \\
\hline$(0,1 ; 5 ; 1)$ & $\mathrm{KR}$ & 0,893 & 0,866 & 0,049 & 0,764 & 0,669 & 0,050 & 0,626 & 0,556 & 0,059 \\
\hline$(0,5 ; 0,1 ; 1)$ & $\mathrm{C}$ & 0,914 & 0,885 & 0,053 & 0,788 & 0,692 & 0,055 & 0,723 & 0,658 & 0,051 \\
\hline$(0,5 ; 0,1 ; 1)$ & $\mathrm{R}$ & 0,930 & 0,901 & 0,060 & 0,825 & 0,724 & 0,065 & 0,779 & 0,701 & 0,065 \\
\hline$(0,5 ; 0,1 ; 1)$ & $\mathrm{S}$ & 0,917 & 0,893 & 0,057 & 0,804 & 0,710 & 0,059 & 0,755 & 0,684 & 0,057 \\
\hline$(0,5 ; 0,1 ; 1)$ & $\mathrm{KR}$ & 0,916 & 0,891 & 0,055 & 0,800 & 0,706 & 0,058 & 0,746 & 0,678 & 0,052 \\
\hline$(0,1 ; 0,5 ; 1)$ & $\mathrm{C}$ & 0,915 & 0,884 & 0,052 & 0,819 & 0,716 & 0,058 & 0,702 & 0,639 & 0,048 \\
\hline$(0,1 ; 0,5 ; 1)$ & $\mathrm{R}$ & 0,931 & 0,896 & 0,062 & 0,854 & 0,756 & 0,072 & 0,759 & 0,691 & 0,073 \\
\hline$(0,1 ; 0,5 ; 1)$ & $\mathrm{S}$ & 0,919 & 0,887 & 0,054 & 0,832 & 0,732 & 0,063 & 0,730 & 0,668 & 0,058 \\
\hline$(0,1 ; 0,5 ; 1)$ & $\mathrm{KR}$ & 0,918 & 0,887 & 0,053 & 0,829 & 0,726 & 0,059 & 0,722 & 0,654 & 0,052 \\
\hline$(1 ; 1 ; 1)$ & $\mathrm{C}$ & 0,906 & 0,875 & 0,053 & 0,809 & 0,715 & 0,054 & 0,690 & 0,635 & 0,052 \\
\hline$(1 ; 1 ; 1)$ & $\mathrm{R}$ & 0,923 & 0,892 & 0,060 & 0,844 & 0,741 & 0,075 & 0,747 & 0,685 & 0,063 \\
\hline$(1 ; 1 ; 1)$ & $\mathrm{S}$ & 0,909 & 0,876 & 0,053 & 0,819 & 0,719 & 0,058 & 0,713 & 0,649 & 0,055 \\
\hline$(1 ; 1 ; 1)$ & $\mathrm{KR}$ & 0,908 & 0,875 & 0,053 & 0,815 & 0,718 & 0,053 & 0,704 & 0,640 & 0,054 \\
\hline
\end{tabular}

* C:Containment, R:Residual, S: Satterthwaite e KR: Kenward-Roger.

Observa-se na maioria dos casos, dentro dos valores de cada variação e de cada nível de desbalanceamento, que o poder do teste foi maior quando a taxa de erro tipo I foi maior. Também nota-se que com o aumento na porcentagem de desbalanceamento o poder o teste diminui e a taxa de erro tipo I aumentou, assim houve um melhor controle da taxa de erro tipo I para o menor grau de desbalanceamento.

Observa-se também, dentro de cada nível de desbalanceamento, que os valores do poder do teste ficaram mais semelhantes entre si, bem como os valores da taxa de erro tipo I, do que os valores apresentados nas Tabelas 6 para $n=30$ e 10 para $n=60$. No presente cenário o poder do teste foi maior e o controle da taxa de erro tipo I foi melhor do que as situações apresentadas nas Tabelas 6 e 10. 
$D B C / P S D$ com $n=120$ - fator $A$

Na Tabela 15 nota-se que o método Residual apresentou maior poder de teste para todas as situações, porém esse método apresentou maior taxa de erro tipo I em comparação aos demais métodos.

Tabela 15 - Poder analítico (P.A.), poder simulado (P.S.) e taxa de erro tipo I $(\hat{\alpha})$ para o fator $A$ no Modelo VI com desbalanceamentos de $10 \%, 20 \%$ e $30 \%$

\begin{tabular}{|c|c|c|c|c|c|c|c|c|c|c|}
\hline \multirow{2}{*}{$\begin{array}{c}\text { Variação } \\
\left(\sigma_{B l}^{2}, \sigma_{P}^{2}, \sigma_{R}^{2}\right)\end{array}$} & \multirow{2}{*}{ Método* } & \multicolumn{3}{|c|}{$10 \%$} & \multicolumn{3}{|c|}{$20 \%$} & \multicolumn{3}{|c|}{$30 \%$} \\
\hline & & P.A. & P.S. & $\hat{\alpha}$ & P.A. & P.S. & $\hat{\alpha}$ & P.A. & P.S. & $\hat{\alpha}$ \\
\hline$(0,1 ; 5 ; 1)$ & $\mathrm{C}$ & 0,060 & 0,058 & 0,049 & 0,654 & 0,072 & 0,054 & 0,128 & 0,120 & 0,050 \\
\hline$(0,1 ; 5 ; 1)$ & $\mathrm{R}$ & 0,060 & 0,069 & 0,058 & 0,066 & 0,078 & 0,067 & 0,133 & 0,136 & 0,059 \\
\hline$(0,1 ; 5 ; 1)$ & S & 0,060 & 0,058 & 0,050 & 0,066 & 0,074 & 0,059 & 0,130 & 0,122 & 0,052 \\
\hline$(0,1 ; 5 ; 1)$ & $\mathrm{KR}$ & 0,060 & 0,058 & 0,050 & 0,066 & 0,072 & 0,058 & 0,130 & 0,122 & 0,051 \\
\hline$(0,5 ; 0,1 ; 1)$ & $\mathrm{C}$ & 0,372 & 0,318 & 0,039 & 0,530 & 0,456 & 0,036 & 0,533 & 0,440 & 0,026 \\
\hline$(0,5 ; 0,1 ; 1)$ & $\mathrm{R}$ & 0,395 & 0,348 & 0,045 & 0,559 & 0,489 & 0,042 & 0,563 & 0,484 & 0,030 \\
\hline$(0,5 ; 0,1 ; 1)$ & S & 0,372 & 0,326 & 0,039 & 0,530 & 0,463 & 0,037 & 0,537 & 0,446 & 0,027 \\
\hline$(0,5 ; 0,1 ; 1)$ & $\mathrm{KR}$ & 0,371 & 0,325 & 0,039 & 0,523 & 0,453 & 0,036 & 0,530 & 0,438 & 0,024 \\
\hline$(0,1 ; 0,5 ; 1)$ & $\mathrm{C}$ & 0,166 & 0,139 & 0,047 & 0,231 & 0,202 & 0,044 & 0,270 & 0,227 & 0,040 \\
\hline$(0,1 ; 0,5 ; 1)$ & $\mathrm{R}$ & 0,175 & 0,166 & 0,058 & 0,244 & 0,232 & 0,051 & 0,286 & 0,241 & 0,048 \\
\hline$(0,1 ; 0,5 ; 1)$ & S & 0,169 & 0,140 & 0,048 & 0,236 & 0,206 & 0,045 & 0,276 & 0,226 & 0,041 \\
\hline$(0,1 ; 0,5 ; 1)$ & $\mathrm{KR}$ & 0,169 & 0,139 & 0,048 & 0,235 & 0,201 & 0,044 & 0,275 & 0,217 & 0,038 \\
\hline$(1 ; 1 ; 1)$ & $\mathrm{C}$ & 0,099 & 0,084 & 0,048 & 0,132 & 0,125 & 0,046 & 0,181 & 0,162 & 0,040 \\
\hline$(1 ; 1 ; 1)$ & $\mathrm{R}$ & 0,103 & 0,095 & 0,058 & 0,137 & 0,141 & 0,053 & 0,190 & 0,181 & 0,047 \\
\hline$(1 ; 1 ; 1)$ & S & 0,099 & 0,084 & 0,050 & 0,132 & 0,125 & 0,045 & 0,182 & 0,164 & 0,040 \\
\hline$(1 ; 1 ; 1)$ & $\mathrm{KR}$ & 0,099 & 0,084 & 0,048 & 0,131 & 0,123 & 0,044 & 0,181 & 0,160 & 0,038 \\
\hline
\end{tabular}

${ }^{*}$ C:Containment, R:Residual, S: Satterthwaite e KR: Kenward-Roger.

Na maioria dos casos, os métodos de Containment e Kenward-Roger controlaram melhor a taxa de erro tipo I do que os métodos de Satterthwaite e Residual, no entanto o poder de teste desses dois últimos métodos foi superior aos outros dois, sendo que para todos os casos o método de Kenward-Roger apresentou o poder do teste maior do que o método de Containment. Nota-se também que em todos os casos o método de Satterthwaite produziu um poder do teste maior do que o método de Kenward-Roger, porém esse último método controlou melhor a taxa de erro tipo I.

Observa-se que o poder do teste foi maior quando a variância da parcela, $\sigma_{P}^{2}$, foi menor que a variância residual, $\sigma_{R}^{2}$, e que a taxa de erro tipo I foi melhor controlada 
quando a variância da parcela foi menor. Nota-se que na maioria dos casos dentro dos valores de cada variação e de cada nível de desbalanceamento, o poder do teste foi maior quando a taxa de erro tipo I foi maior. Também nota-se que na maioria dos casos com o aumento na porcentagem de desbalanceamento a taxa de erro tipo I diminuiu e o poder o teste aumentou, porém houve um melhor controle da taxa de erro tipo I para o maior grau de desbalanceamento.

Observa-se também, dentro de cada nível de desbalanceamento, que os valores do poder do teste ficaram mais semelhantes entre si, bem como os valores da taxa de erro tipo I, do que os valores apresentados nas Tabelas 7 para $n=30$ e 11 para $n=60$. No presente cenário o poder do teste foi maior e o controle da taxa de erro tipo I foi melhor do que as situações apresentadas nas Tabelas 7 e 11.

$D B C / P S D \operatorname{com} n=120$ - fator $B$

Na Tabela 16 nota-se que o método Residual apresentou maior poder de teste para todas as situações, porém esse método apresentou maior taxa de erro tipo I em comparação aos demais métodos.

Em alguns casos os métodos de Containment e Kenward-Roger controlaram melhor a taxa de erro tipo I do que os métodos de Satterthwaite e Residual, no entanto o poder de teste desses dois últimos métodos foi superior aos outros dois, sendo que para alguns casos o método de Kenward-Roger apresentou o poder do teste maior do que o método de Containment. Nota-se também que em alguns casos o método de Satterthwaite produziu um poder do teste maior do que o método de Kenward-Roger, porém esse último método controlou melhor a taxa de erro tipo I. Mas o que ficou evidente na Tabela 16 é que os quatro métodos apresentaram os valores do poder do teste, tanto simulado como o analítico, e a taxa de erro tipo I muito próximos entre si.

Observa-se na maioria dos casos que o poder do teste foi maior quando a variância da parcela, $\sigma_{P}^{2}$, foi menor que a variância residual, $\sigma_{R}^{2}$, e que houve um melhor controle da taxa de erro tipo I no maior grau de desbalanceamento e o poder do teste foi maior no menor grau de desbalanceamento. Nota-se na maioria dos casos, dentro dos valores de cada variação e de cada nível de desbalanceamento, que o poder do teste é maior quando a taxa de erro tipo I é maior.

Observa-se também, dentro de cada nível de desbalanceamento, que os valores do poder do teste ficaram mais semelhantes entre si, bem como os valores da taxa de 
Tabela 16 - Poder analítico (P.A.), poder simulado (P.S.) e taxa de erro tipo I $(\hat{\alpha})$ para o fator $B$ no Modelo VI com desbalanceamentos de $10 \%, 20 \%$ e $30 \%$

\begin{tabular}{|c|c|c|c|c|c|c|c|c|c|c|}
\hline \multirow{2}{*}{$\begin{array}{c}\text { Variação } \\
\left(\sigma_{B l}^{2}, \sigma_{P}^{2}, \sigma_{R}^{2}\right)\end{array}$} & \multirow{2}{*}{ Método* } & \multicolumn{3}{|c|}{$10 \%$} & \multicolumn{3}{|c|}{$20 \%$} & \multicolumn{3}{|c|}{$30 \%$} \\
\hline & & P.A. & P.S. & $\hat{\alpha}$ & P.A. & P.S. & $\hat{\alpha}$ & P.A. & P.S. & $\hat{\alpha}$ \\
\hline$(0,1 ; 5 ; 1)$ & $\mathrm{C}$ & 0,064 & 0,070 & 0,060 & 0,065 & 0,061 & 0,051 & 0,061 & 0,053 & 0,040 \\
\hline$(0,1 ; 5 ; 1)$ & $\mathrm{R}$ & 0,064 & 0,077 & 0,064 & 0,065 & 0,075 & 0,053 & 0,062 & 0,058 & 0,049 \\
\hline$(0,1 ; 5 ; 1)$ & S & 0,063 & 0,070 & 0,061 & 0,065 & 0,063 & 0,051 & 0,061 & 0,054 & 0,040 \\
\hline$(0,1 ; 5 ; 1)$ & $\mathrm{KR}$ & 0,064 & 0,070 & 0,060 & 0,065 & 0,060 & 0,051 & 0,061 & 0,053 & 0,040 \\
\hline$(0,5 ; 0,1 ; 1)$ & $\mathrm{C}$ & 0,077 & 0,081 & 0,058 & 0,060 & 0,065 & 0,051 & 0,068 & 0,063 & 0,055 \\
\hline$(0,5 ; 0,1 ; 1)$ & $\mathrm{R}$ & 0,077 & 0,083 & 0,058 & 0,060 & 0,073 & 0,062 & 0,069 & 0,079 & 0,060 \\
\hline$(0,5 ; 0,1 ; 1)$ & S & 0,077 & 0,081 & 0,058 & 0,060 & 0,071 & 0,057 & 0,068 & 0,073 & 0,058 \\
\hline$(0,5 ; 0,1 ; 1)$ & $\mathrm{KR}$ & 0,077 & 0,081 & 0,058 & 0,060 & 0,067 & 0,055 & 0,068 & 0,063 & 0,055 \\
\hline$(0,1 ; 0,5 ; 1)$ & $\mathrm{C}$ & 0,068 & 0,072 & 0,060 & 0,063 & 0,070 & 0,044 & 0,070 & 0,067 & 0,044 \\
\hline$(0,1 ; 0,5 ; 1)$ & $\mathrm{R}$ & 0,069 & 0,075 & 0,062 & 0,063 & 0,075 & 0,051 & 0,071 & 0,075 & 0,054 \\
\hline$(0,1 ; 0,5 ; 1)$ & S & 0,068 & 0,072 & 0,060 & 0,063 & 0,071 & 0,048 & 0,070 & 0,070 & 0,046 \\
\hline$(0,1 ; 0,5 ; 1)$ & $\mathrm{KR}$ & 0,068 & 0,072 & 0,060 & 0,063 & 0,069 & 0,043 & 0,070 & 0,068 & 0,043 \\
\hline$(1 ; 1 ; 1)$ & $\mathrm{C}$ & 0,066 & 0,072 & 0,061 & 0,057 & 0,051 & 0,046 & 0,057 & 0,059 & 0,044 \\
\hline$(1 ; 1 ; 1)$ & $\mathrm{R}$ & 0,067 & 0,072 & 0,066 & 0,057 & 0,068 & 0,052 & 0,058 & 0,063 & 0,052 \\
\hline$(1 ; 1 ; 1)$ & S & 0,066 & 0,072 & 0,061 & 0,057 & 0,056 & 0,049 & 0,057 & 0,059 & 0,046 \\
\hline$(1 ; 1 ; 1)$ & $\mathrm{KR}$ & 0,066 & 0,072 & 0,061 & 0,057 & 0,052 & 0,046 & 0,057 & 0,059 & 0,043 \\
\hline
\end{tabular}

${ }^{*}$ C:Containment, R:Residual, S: Satterthwaite e KR: Kenward-Roger.

erro tipo I, do que os valores apresentados nas Tabelas 8 para $n=30$ e 12 para $n=60$. No presente cenário o poder do teste foi maior e o controle da taxa de erro tipo I foi melhor do que as situações apresentadas nas Tabelas 8 e 12 .

$D B C / P S D$ com $n=120$ - fator $B$ fixo e fator $A$ aleatório

Na Tabela 17 nota-se que o método Residual apresentou maior poder de teste para todas as situações, porém esse método apresentou maior taxa de erro tipo I em comparação aos demais métodos.

Para todos os casos o método de Containment apresentou a taxa de erro tipo I severamente tendenciosa e o poder de teste simulado bem menor do que o poder simulado dos outros métodos, já o método de Kenward-Roger foi o que melhor controlou a taxa de erro tipo I. 
Os métodos de Satterthwaite e Kenward-Roger apresentaram os valores do poder do teste, tanto analítico quanto o simulado, e a taxa de erro tipo I próximos entre si, porém em alguns casos o método de Kenward-Roger controlou melhor a taxa de erro tipo I.

Observa-se na maioria dos casos que o poder do teste foi maior quando a variância da parcela, $\sigma_{P}^{2}$, foi menor que a variância residual, $\sigma_{R}^{2}$, e que dentro dos valores de cada variação e de cada nível de desbalanceamento, o poder do teste é maior quando a taxa de erro tipo I é maior.

Tabela 17 - Poder analítico (P.A.), poder simulado (P.S.) e taxa de erro tipo I ( $\hat{\alpha})$ para o fator $B$ no Modelo IX, considerando $\sigma_{A}^{2}=\sigma_{A B}^{2}=0,1$, com desbalanceamentos de $10 \%, 20 \%$ e $30 \%$

\begin{tabular}{|c|c|c|c|c|c|c|c|c|c|c|}
\hline \multirow{2}{*}{$\begin{array}{c}\text { Variação } \\
\left(\sigma_{B l}^{2}, \sigma_{P}^{2}, \sigma_{R}^{2}\right)\end{array}$} & \multirow{2}{*}{ Métodos* } & \multicolumn{3}{|c|}{$10 \%$} & \multicolumn{3}{|c|}{$20 \%$} & \multicolumn{3}{|c|}{$30 \%$} \\
\hline & & P.A. & P.S. & $\hat{\alpha}$ & P.A. & P.S. & $\hat{\alpha}$ & P.A. & P.S. & $\hat{\alpha}$ \\
\hline$(0,1 ; 5 ; 1)$ & $\mathrm{C}$ & 0,151 & 0,030 & 0,006 & 0,086 & 0,015 & 0,006 & 0,127 & 0,032 & 0,006 \\
\hline$(0,1 ; 5 ; 1)$ & $\mathrm{R}$ & 0,216 & 0,145 & 0,037 & 0,109 & 0,086 & 0,050 & 0,175 & 0,142 & 0,040 \\
\hline$(0,1 ; 5 ; 1)$ & $\mathrm{S}$ & 0,212 & 0,112 & 0,030 & 0,083 & 0,066 & 0,037 & 0,128 & 0,106 & 0,031 \\
\hline$(0,1 ; 5 ; 1)$ & $\mathrm{KR}$ & 0,212 & 0,112 & 0,030 & 0,082 & 0,064 & 0,035 & 0,125 & 0,102 & 0,030 \\
\hline$(0,5 ; 0,1 ; 1)$ & $\mathrm{C}$ & 0,151 & 0,037 & 0,003 & 0,093 & 0,018 & 0,006 & 0,117 & 0,025 & 0,006 \\
\hline$(0,5 ; 0,1 ; 1)$ & $\mathrm{R}$ & 0,216 & 0,161 & 0,040 & 0,119 & 0,096 & 0,049 & 0,159 & 0,112 & 0,043 \\
\hline$(0,5 ; 0,1 ; 1)$ & $\mathrm{S}$ & 0,213 & 0,129 & 0,035 & 0,117 & 0,087 & 0,037 & 0,156 & 0,101 & 0,030 \\
\hline$(0,5 ; 0,1 ; 1)$ & $\mathrm{KR}$ & 0,212 & 0,128 & 0,035 & 0,116 & 0,087 & 0,037 & 0,153 & 0,100 & 0,030 \\
\hline$(0,1 ; 0,5 ; 1)$ & $\mathrm{C}$ & 0,159 & 0,038 & 0,004 & 0,100 & 0,018 & 0,005 & 0,126 & 0,026 & 0,008 \\
\hline$(0,1 ; 0,5 ; 1)$ & $\mathrm{R}$ & 0,229 & 0,164 & 0,036 & 0,132 & 0,092 & 0,048 & 0,173 & 0,118 & 0,041 \\
\hline$(0,1 ; 0,5 ; 1)$ & $\mathrm{S}$ & 0,225 & 0,132 & 0,031 & 0,129 & 0,077 & 0,038 & 0,133 & 0,099 & 0,029 \\
\hline$(0,1 ; 0,5 ; 1)$ & $\mathrm{KR}$ & 0,225 & 0,131 & 0,031 & 0,128 & 0,076 & 0,035 & 0,129 & 0,093 & 0,029 \\
\hline$(1 ; 1 ; 1)$ & $\mathrm{C}$ & 0,160 & 0,039 & 0,005 & 0,094 & 0,017 & 0,003 & 0,134 & 0,027 & 0,008 \\
\hline$(1 ; 1 ; 1)$ & $\mathrm{R}$ & 0,230 & 0,161 & 0,039 & 0,121 & 0,088 & 0,042 & 0,186 & 0,129 & 0,040 \\
\hline$(1 ; 1 ; 1)$ & S & 0,227 & 0,130 & 0,032 & 0,119 & 0,073 & 0,033 & 0,138 & 0,107 & 0,031 \\
\hline$(1 ; 1 ; 1)$ & $\mathrm{KR}$ & 0,226 & 0,130 & 0,031 & 0,118 & 0,072 & 0,032 & 0,133 & 0,101 & 0,028 \\
\hline
\end{tabular}

* C:Containment, R:Residual, S: Satterthwaite e KR: Kenward-Roger.

Observa-se também, dentro de cada nível de desbalanceamento, que os valores do poder do teste para os métodos de Containment, Satterthwaite e Kenward-Roger ficaram mais semelhantes entre si, bem como os valores da taxa de erro tipo I, do que os valores apresentados na Tabela 9 para $n=30$ e Tabela 13 para $n=60$. No presente 
cenário o poder do teste foi maior e o controle da taxa de erro tipo I foi melhor do que as situações apresentadas nas Tabelas 9 e 13.

Entre os autores que já escreveram sobre os métodos de cálculo do número aproximado de graus de liberdade tem-se Piepho (1997) que encontrou um razoável desempenho do método de Satterthwaite considerando um experimento com subamostras no delineamento casualizado em blocos com dados desbalanceados. Kenward e Roger (1997) obtiveram um bom desempenho de seu método verificando-o por meio de simulações. Os resultados desse trabalho estão de acordo com os resultados desses autores. Schaalje, McBride, e Felling-ham (2002) investigaram experimentos de medidas repetidas, com cinco estruturas de covariância e por meio de estudos de simulação, constataram que o método de Kenward-Roger funcionou tão bem quanto ou melhor do que o método de Satterthwaite em todas as situações, produziu taxas de erro do tipo I próximas dos valores nominais nos casos das estruturas de variância de simetria composta e Toeplitz. Ainda nesse estudo, quando a estrutura de variância e covariância veio a ser mais complexa, mesmo o método de Kenward-Roger teve problemas e produziu taxas de erro inflacionadas. Para todos os cenários analisados nesse trabalho, tanto o método de Kenward-Roger como o de Satterthwaite controlaram bem a taxa de erro tipo I sem perder no poder do teste. 


\section{CONCLUSÕES}

Os resultados obtidos nesse estudo permitem as seguintes conclusões:

- Nos modelos com um fator de tratamento e delineamento casualizado em blocos, os métodos Containment, Residual, Satterthwaite e Kenward-Roger apresentaram um controle satisfatório da taxa de erro tipo I;

- Nos modelos com tratamentos no esquema de parcelas subdivididas e delineamento casualizado em blocos, considerando o bloco de efeito aleatório, os métodos Containment, Satterthwaite e Kenward-Roger apresentaram um controle satisfatório da taxa de erro tipo I, já o método Residual apresentou maior taxa de erro tipo I e maior poder de teste;

- Nos modelos com tratamentos no esquema de parcelas subdivididas e delineamento casualizado em blocos, com os fatores bloco e tratamento de efeitos aleatórios, o método Containment apresentou valores muito baixos para o poder de teste e a taxa de erro tipo I, já os métodos de Satterthwaite e Kenward-Roger proporcionaram um melhor controle na taxa de erro tipo I, enquanto que o método Residual apresentou maior taxa de erro tipo I;

- Tanto nos modelos com um fator de tratamentos como nos modelos com tratamentos no esquema de parcelas subdivididas, o controle da taxa de erro tipo I, e o aumento do poder do teste, foram mais expressivos com o aumento do tamanho da amostra;

- Nos cenários em que a variância residual foi relativamente pequena obteve-se menores valores de poder do teste do que em cenários com variância residual relativamente grande;

- Na maioria dos casos dentro das situações estudadas o aumento no poder do teste acompanhou o aumento na taxa de erro tipo I. Assim, as diferenças de poder entre os métodos analisados foram devidas, principalmente, às diferenças no controle do erro tipo I. 


\section{REFERÊNCIAS}

ALNOSAIER, W.S. Kenward-Roger Approximate F Test for Fixed Effects in Mixed Linear Models. 2007. 132 p. Tese (Doutorado em Estatística) - Oregon State University, Oregon, 2007.

ALCARDE, R. Modelos lineares mistos em dados longitudionais com o uso do pacote ASReml-R. 2012. 156 p. Tese (Doutorado em Estatística e Experimentação Agronômica) - Escola Superior de Agricultura "Luiz de Queiroz", Universidade de São Paulo, Piracicaba, 2012.

ANDERSON, R.L.; BANCROFT T.A. Statistical Theory in Research. New York: McGraw-Hill Book Company, 1952. 399 p.

ANDRADE, D.F. de; OGLIARI, P.J. Estatística para as ciências agrárias e biológicas: com noções de experimentação. Florianópolis: UFSC, 2013. 438 p.

BERNDTSON, W.E.A. Simple, rapid and reliable method for selecting or assessing the number of replicates for animal experiments. Journal of animal science, Champaign, v. 69, n. 1, p. 67-76, 1991.

BINDER, K.; HEERMAnN, D.W. Monte Carlo Methods in Statistical Phyvsics: an introduction. Berlin: Springer-Verlag, 2010, 202 p.

CASELLA, G.; BERGER, R.L. Statistical Inference. Pacific Grove: Thomson Learning, 2002. $686 \mathrm{p}$.

COHEN, J. Statistical Power Analysis for the Behavioral Sciences. New Jersey: Hillsdale, 1988. 567 p.

Some statistical issues in psychological research. New York: McGraw-Hill, 1965. $260 \mathrm{p}$.

COUSEnS, R.; MARSHALL, C. Dangers in testing statistical hypotheses. Annals of Applied Biology, Hoboken, v. 111, n. 2, p. 469-476, 1987.

FISHER, R.A. The Correlation Between Relatives on the Supposition of Mendelian Inheritance. Earth and Environmental Science Trans2000. actions of the Royal Society of Edinburgh, Cambridge, v. 52, n. 2, p. 399-433, 1918.

Statistical methods for research workers. Oliver and Boyd: Edinburgh and London, 1925. $162 \mathrm{p}$.

GILMOUR, A.R.; THOMPSON, R.; CULLIS, B.R. Average information reml: an efficient algorithm for variance parameter estimation in linear mixed models. Biometrics, Arlington, v. 51, n. 4, p. 1440-1450, 1995.

GUIARD, V.; SPILKE J.; DÄNICKE S. Evaluation and interpretation of results for three cross-over designs. Archive of Animal Nutrition, London, v. 57, n. 3, p. 177-195, 2003.

HARTLEY, H.O.; RAO, J.N.K. Maximum-likelihood estimation for the mixed analysis of variance model. Biometrika, Oxford, v. 54, n. 2, p. 93-108, 1967. 
HARVILLE, D.A. Maximum likelihood approaches to variance component estimation and to related problems. Journal of the American Statistical Association, Alexandria, v. 72, n. 358, p. $320-340,1977$.

HARVILLE, D.A.; CARRIQUIRY, A.L. Classical and Bayesian Prediction as Applied to an Unbalanced Mixed Linear Model. Biometrics, Hoboken, v. 48, n. 4, p. 987-1003, 1992.

HARVILLE, D.A.; JESKE, D.R. Mean Squared Error of Estimation or Prediction Under a General Linear Model. Journal of the American Sataistical Association, Alexandria, v. 87, n. 419, p. 724-731, 1992.

HELMS, R.W. Intentionally incomplete longitudinal designs: Methodology and comparison of some full span designs. Statistics in Medicine, Chichester, v. 11, p. 1889-1913, 1992.

HENDERSON, C.R. Estimation of variance and variance components. Biometrics, Hoboken, v. 9, n. 2, p. 226-252, 1953.

Selection index and expected genetic advance. In: HANSON, W. D.; ROBINSON, H. F. (Ed.). Statistical genetics and plant breeding. Washington DC: National Academy of Genetic Advance - National Research Council, 1963. p. 141-163.

Statistical Method in Animal Improvement: Historical Overview. In: GIANOLA, D.; HAMMOND, K. Advances in Statistical Methods for Genetic improvement of Livestock, New York: Springer, 1990. cap. 1, p. 2-14.

HULTING, F.L.; HARVILLE, D.A. Some Bayesian and non-Bayesian procedures for the analysis of comparative experiments and for small area estimation: Computational aspects,frequentist properties, and relationships. Journal of the American Statistical Association, Alexandria, v. 86, n. 415, p. 557-568, 1991.

HUYNH, H.; FELDT, L.S. Conditions under which mean square ratios in repeated measurements designs have exact F-distributions. Journal of the American Statistical Association, Alexandria, v. 65, n. 332, p. 1582-1589, 1970.

Estimation of the Box Correction for Degrees of Freedom from Sample Data in Randomized Block and Split-Plot Designs. Journal of Educational Statistics, New York, v. 1, n. 1 , p. $69-82,1976$.

JESKE, D.R.; HARVILLE, D.A. Prediction-interval procedures and (fixed-effects) confidence-interval procedures for mixed linear models. Communications in Statistics Theory and Methods, New York, v. 17, n. 4, p. 1053-1087, 1988.

KACKAR, A.N., HARVILLE, D.A. Unbiasedness of Two-Stage Estimation and Precision Procedures for Mixed Linear Models. Communications in Statistics A, Ontario, v. 10, p. 1249-1261, 1981.

Approximation for Standard Errors of Estimators of Fixed and Random Effects in Mixed Linear Models. Journal of the American Statistical Association, Alexandria, v. 79, n. 388, p. $853-861,1984$.

KENWARD, M.G.; ROGER, J.H. Small Sample Inference for Fixed Effects from Restricted Maximum Likelihood. Biometrics, Hoboken, v. 53, n. 3, p. 983-997, 1997. 
KHURI, A.I.; MATHEW, T.; SINHA, B.K. Statistical tests for mixed linear models. New York: John Wiley \& Sons, 1998. 384 p.

KRZANOWSKI, W.J. Principles of Multivariate Analysis. Reino Unido: Oxford University Press, 2000. 608 p.

LITTELL, R.C.; MILLIKEN, G.A.; STROUP, W.W.; WOLFINGER, R.D.; SCHABENBERGER, O. SAS ${ }^{\circledR}$ for Mixed Models. 2.ed. Cary, NC: SAS Institute Inc. 2006. $814 \mathrm{p}$.

PATTERSON, H.D.; THOMPSON, R. Recovery of inter-block information when block sizes are unequal. Biometrika, Oxford, v. 58, n. 3, p. 545-554, 1971.

PIEPHO, H.P. Analysis of a Randomized Complete Block Design with Unequal Subclass Numbers. Agronomy Journal, Madison, v. 89, n. 5, p. 718-723, 1997.

PIEPHO, H.P.; SPILKE J. Anmerkungen zur Analyse balancierten gemischter Modelle mit der SAS-Prozedur MIXED. Zeitschrift für Agrarinformatik, Altensteig, v. 7, n. 2, p. 39-46, 1999.

PERRI, S.H.V.; IEMMA, A.F. Procedimento "MIXED" do SAS para análise de modelos mistos. Scientia Agricola, Piracicaba, v. 56, n. 4, p. 959-967, 1999.

PRASAD, N.; RAO, J. The Estimation of the Mean Squared Error of Small-Area Estimators. Journal of the American Statistical Association, Alexandria, v. 85, n. 409, p. 163-171, 1990.

RAO, C.R. Estimation of variance and covariance components - MINQUE Theory. Journal of Multivariate Analysis, San Diego, v. 1, n. 3, p. 257-275, 1971a.

Minimum variance quadratic unbiased estimation of variance components. Journal of Multivariate Analysis, San Diego, v. 1, n. 3, p. 445-456, $1971 b$.

ROBINSON, G.K. That BLUP is a good-thing: The estimation of random effects. Statistical Science, Cleveland, v. 6, n. 1, p. 15-51, 1991.

ROSA, G.J.M.; STEIBEL, J.P.; TEMPELMAN, R.J. Reassessing Design and Analysis of Two-Colour Microarray Experiments Using Mixed Effects Models. Comparative and Functional Genomics, Oxford, v. 6, n. 3, p. 123-131, 2005.

RUSSELL, T.S.; BRADLEY, R.A. One-Way Variances in a Two-Way Classification, Biometrika, Oxford, v. 45, n. 2, p. 111-129, 1958.

SAS INSTITUTE INC. SAS OnlineDoc ${ }^{\circledR}$ 9.1.3. Cary, 2004. Disponível em: <http://support.sas.com/onlinedoc/913/docMainpage.jsp>. Acesso em: 30 nov. 2014.

SAS OnlineDoc ${ }^{\circledR}$ 13.1. Cary, 2013. Disponível em:

$<$ http://support.sas.com/documentation/onlinedoc/stat/131/mixed.pdf >. Acesso em: 30 nov. 2014.

SATTERTHWAITE, F.E. Synthesis of Variance. Psychometrika, New York, v. 6, n. 5, 1941, p. 309-316.

An approximate distribution of estimates of variance components. Biometrics, Hoboken, v. 2, n. 6, p. 110-114, 1946. 
SCHAALJE, G.B.; MCBRIDE, J.B.; FELLINGHAM, G.W. Adequacy of Approximation to Distributions of Test Statistics in Complex Mixed Linear Models. Journal of Agricultural, Biological, and Environmental Statistics, London, v. 7, n. 4, p. 512-524, 2002.

SEARLE, S.R. Topics in Variance Component Estimation. Biometrics, Hoboken, v. 27, n. 1, p. 1-76, 1971.

Linear models for unbalanced data. New York: John Wiley \& Sons, 1987. 536

p.

SEARLE, S.R.; CASELLA, G.; MCCULLOCH, C.E. Variance components. New York: John Wiley, 1992. p. 536.

SPILKE, J.; PIEPHO, H.P.; HU, X. Analysis of Unbalanced Data by Mixed Linear Models Using the MIXED Procedure of the SAS System. Journal of Agronomy and Crop Science, Hoboken, v. 191, n. 1, p. 47-54, 2005.

SPILKE, J.; TUCHSCHERER, A. Simulationsuntersuchungen zum Einfluss verschiedener Strategien der Varianzkomponentenschätzung und Hypothesenprüfung auf die statistischen Risiken in gemischten linearen Modellen mit ungleicher Klassenbesetzung. Zeitschrift für Agrarinformatik, Altensteig, v. 10, n. 2, p. 66-75, 2001.

STEEL, R.G.D.; TORRIE, J.H. Principles and procedures of statistics: a biometrical approach. New York: Mc-Graw Hill, 1981. 633 p.

STROUP, W.W. Power Analysis Based on Spatial Effects Mixed Models: A Tool for Comparing Design and Analysis Strategies in the Presence of Spatial Variability. Journal of Agricultural, Biological, and Environmental Statistics, London, v. 7, n. 4, p. 491-511, 2002.

TEMPELMAN, R.J. Assessing Statistical Precision, Power, and Robustness of Alternative Experimental Designs for Two Colour Microarray Platforms Based on Mixed Effects Models. Veterinary Immunology and Immunopathology, Amsterdam, v. 105, p. 175-186, 2005.

THOMPSON, R. Maximum likelihood estimation of variance components. Mathematische Operationsforschung und Statistik, Series Statistics, London, v. 11, p. 545-561, 1980.

THOMPSON, W.A., Jr. The Problem of Negative Estimates of Variance Components.

Annals of Mathematical Statistics, Hoboken, v. 33, n. 1, p. 273-289, 1962.

VERBEKE, G.; MOLENBERGHS, G. Linear mixed models for longitudinal data. New York: Springer-Verlag, 2000. 568 p.

ZIMMERMANN, F.J.P. Estatística aplicada à pesquisa agrícola. Santo Antônio de Goiás: Embrapa Arroz e Feijão, 2004. 400 p. 
ANEXOS 


\section{ANEXO A - Programação SAS}

*Delineamento casualizado em blocos para $n=30$, com $10 \%$ de observacoes perdidas;

$\%$ let seed $=8402698$;

$\%$ let var_resid $=1$;

$\%$ let var_bloco $=0.1$;

$\%$ let nsim $=1000$;

data dadosim;

do bloco $=1$ to 3 ;

u_bloco $=$ sqrt (\&var_bloco $) *$ rannor (\&seed) ;

do trat $=1$ to 10 ;

e_res $=$ sqrt (\&var_resid) $*$ rannor (\&seed);

$\mathrm{y}=\mathrm{u}_{-} \mathrm{bloco}+$ e_res;

if (bloco=1 and trat=2) or (bloco=2 and trat=4) or (bloco=2 and trat=10) then do;

$\mathrm{y}=$.;

end ;

output;

end;

end;

run;

PROC PRINT DATA=dadosim;

RUN;

PROC MIXED Data=dadosim method=REML;

CLASS bloco trat ;

MODEL y = trat/ solution ddfm=contain;

random bloco;

ods output tests $3=$ t 1 ;

LSMEANS trat/diff CL;

RUN ;

*Poder analitico do teste $\mathrm{F}$;

data f_power;

set $t 1$;

Noncen $=$ NumDF $* F V a l u e ;$

Alpha $=0.05$;

FCrit $=$ finv (1-Alpha, NumDF, DenDF, 0$)$;

Power $=1-\operatorname{probf}(F C r i t, N u m D F$, DenDF, Noncen);

run;

proc print data $=f_{-}$power;

run;

*taxa de erro tipo I;

data aleat;

do $\operatorname{sim}=1$ to \&nsim;

do bloco = 1 to 3 ;

$\mathrm{u}_{-} \mathrm{bloco}=\operatorname{sqrt}\left(\& v a r_{-}\right.$bloco $) *$ rannor $(\&$ seed $)$; 
do trat $=1$ to 10 ;

e_res = sqrt (\&var_resid) $*$ rannor (\&seed);

aleat $=$ u_bloco + e_res;

if (bloco=1 and trat=2) or (bloco=2 and trat=4) or (bloco=2 and trat=10) then do; aleat $=$.;

end;

output;

end;

end;

end;

run;

PROC PRINT DATA=aleat;

RUN;

ods exclude all;

ods noresults;

proc mixed data=aleat;

by sim;

class bloco trat;

model aleat=trat /ddfm=contain;

random bloco;

parms \&var_bloco \&var_resid;

ods output covparms=cpsim tests $3=$ t3sim;

run;

ods exclude none;

ods results;

proc sort data=cpsim;

by covparm;

run;

proc means data=cpsim;

by covparm;

var estimate;

run;

data t3sim;

set $\mathrm{t} 3 \mathrm{sim}$;

if probf $<0.05$ then $\operatorname{sig}=1$;

else $\operatorname{sig}=0$;

proc sort data=t3sim;

by effect;

proc means data=t3sim mean;

by effect;

var sig;

run;

*poder do teste simulado;

proc mixed data=dadosim method=REML;

class bloco trat;

model $\mathrm{y}=$ trat/ ddfm=contain outpm=opm;

random bloco;

run; 
PROC PRINT DATA=opm;

RUN;

data opm1;

set opm;

drop y stderrpred df alpha lower upper resid;

run;

PROC PRINT DATA=opm1;

RUN;

proc sort data=aleat;

by bloco trat;

run;

PROC PRINT DATA=aleat;

RUN;

data blocos_sim;

merge aleat opm $1 ; *($ RENAME $=($ trat $=$ trat $\$$ bloco $=$ bloco $\$))$;

by bloco trat;

$\mathrm{y}=$ pred + aleat;

run;

proc print data $=$ blocos_sim;

run;

proc sort data=blocos_sim;

by sim;

run;

ods exclude all;

ods noresults;

proc mixed data=blocos_sim;

by sim;

class bloco trat;

model y = trat /ddfm=contain;

random bloco;

parms \&var_bloco \&var_resid;

ods output covparms=cpsim1 tests $3=t 3 \operatorname{sim} 1$;

run;

ods exclude none;

ods results;

proc sort data $=$ cpsim 1 ;

by covparm;

run;

proc means data $=$ cpsim1;

by covparm;

var estimate;

run;

data t3sim1;

set $t 3 \operatorname{sim} 1$;

if probf $<0.05$ then $\mathrm{sig}=1$;

else $\operatorname{sig}=0$; 
proc sort data=t3sim1;

by effect;

proc means data=t3sim1 mean;

by effect;

var sig;

run;

*Delineamento casualizado em blocos com esquema de tratamentos em parcela subdividida; *para $\mathrm{n}=60$ com $20 \%$ de observacoes perdidas;

$\%$ let seed $=8402698$;

$\%$ let var_resid $=1$;

$\%$ let var_par $=0.5$;

$\%$ let var_bloco $=0.1$;

$\%$ let nsim = 1000;

data dadosim;

do bloco $=1$ to 5 ;

$\mathrm{u}_{-} \mathrm{bloco}=\operatorname{sqrt}\left(\& v a r_{\_} b l o c o\right) *$ rannor $(\&$ seed $)$;

do $A=1$ to 3 ;

e_par $=$ sqrt (\&var_par) $*$ rannor (\&seed);

do $B=1$ to 4 ;

e_sub $_{\text {_s }}$ sqrt (\&var_resid) $*$ rannor (\&seed);

$\mathrm{y}=\mathrm{u}_{-} \mathrm{bloco}+\mathrm{e}_{-}$par $+\mathrm{e}_{-}$sub;

if ( $b l o c o=1$ and $A=2$ and $B=3$ ) or $(b l o c o=4$ and $A=1$ and $B=1)$ or

(bloco=3 and $A=3$ and $B=4$ ) or ( $b l o c o=5$ and $A=1$ and $B=1$ ) or

(bloco=5 and $A=3$ and $B=1$ ) or ( $b l o c o=5$ and $A=3$ and $B=2$ ) or

(bloco=1 and $A=1$ and $B=1$ ) or $(b l o c o=2$ and $A=1$ and $B=1$ ) or

(bloco $=3$ and $A=1$ and $B=3$ ) or $(b l o c o=3$ and $A=3$ and $B=3$ ) or

(bloco=5 and $A=1$ and $B=3$ ) or $(b l o c o=5$ and $A=1$ and $B=4)$ then do;

$\mathrm{y}=$.;

end;

output;

end;

end;

end;

run;

PROC PRINT DATA=dadosim;

RUN;

PROC MIXED Data=dadosim method=REML;

CLASS A B bloco;

MODEL $\mathrm{y}=\mathrm{A} B \mathrm{~A} * \mathrm{~B} /$ solution $\operatorname{ddfm}=r e s i d u a l ;$

random bloco $A * b l o c o$;

ods output tests $3=$ t 1 ;

LSMEANS $A * B /$ diff CL;

RUN ;

* Poder analitico do teste $\mathrm{F}$; 


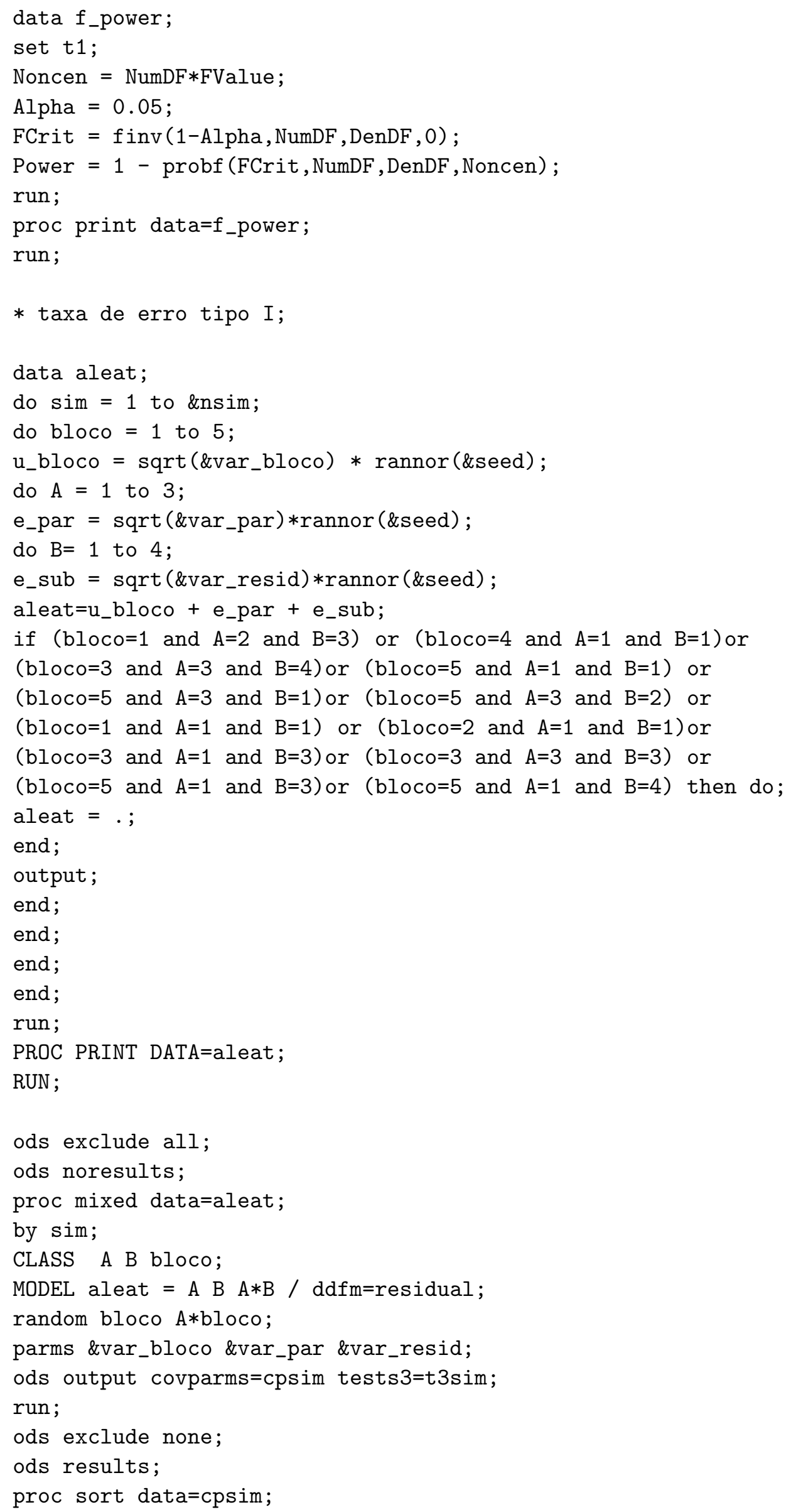


by covparm;

run;

proc means data=cpsim;

by covparm;

var estimate;

run;

data t3sim;

set t3sim;

if probf $<0.05$ then $\mathrm{sig}=1$;

else sig $=0$;

proc sort data=t3sim;

by effect;

proc means data=t3sim mean;

by effect;

var sig;

run;

*poder simulado;

proc mixed data=dadosim method=REML;

CLASS A B bloco;

MODEL $\mathrm{y}=\mathrm{A} B \mathrm{~A} * \mathrm{~B} / \mathrm{ddfm}=$ residual outpm=opm;

random bloco $\mathrm{A} * \mathrm{bloco}$;

run;

PROC PRINT DATA=opm;

RUN;

data opm1;

set opm;

drop y stderrpred df alpha lower upper resid;

run;

PROC PRINT DATA=opm1;

RUN ;

proc sort data=aleat;

by bloco A B;

run;

PROC PRINT DATA=aleat;

RUN;

data blocos_sim;

merge aleat opm1;

by bloco A B;

$\mathrm{y}=$ pred + aleat

run;

proc print data $=$ blocos_sim;

run;

proc sort data=blocos_sim;

by sim;

run; 


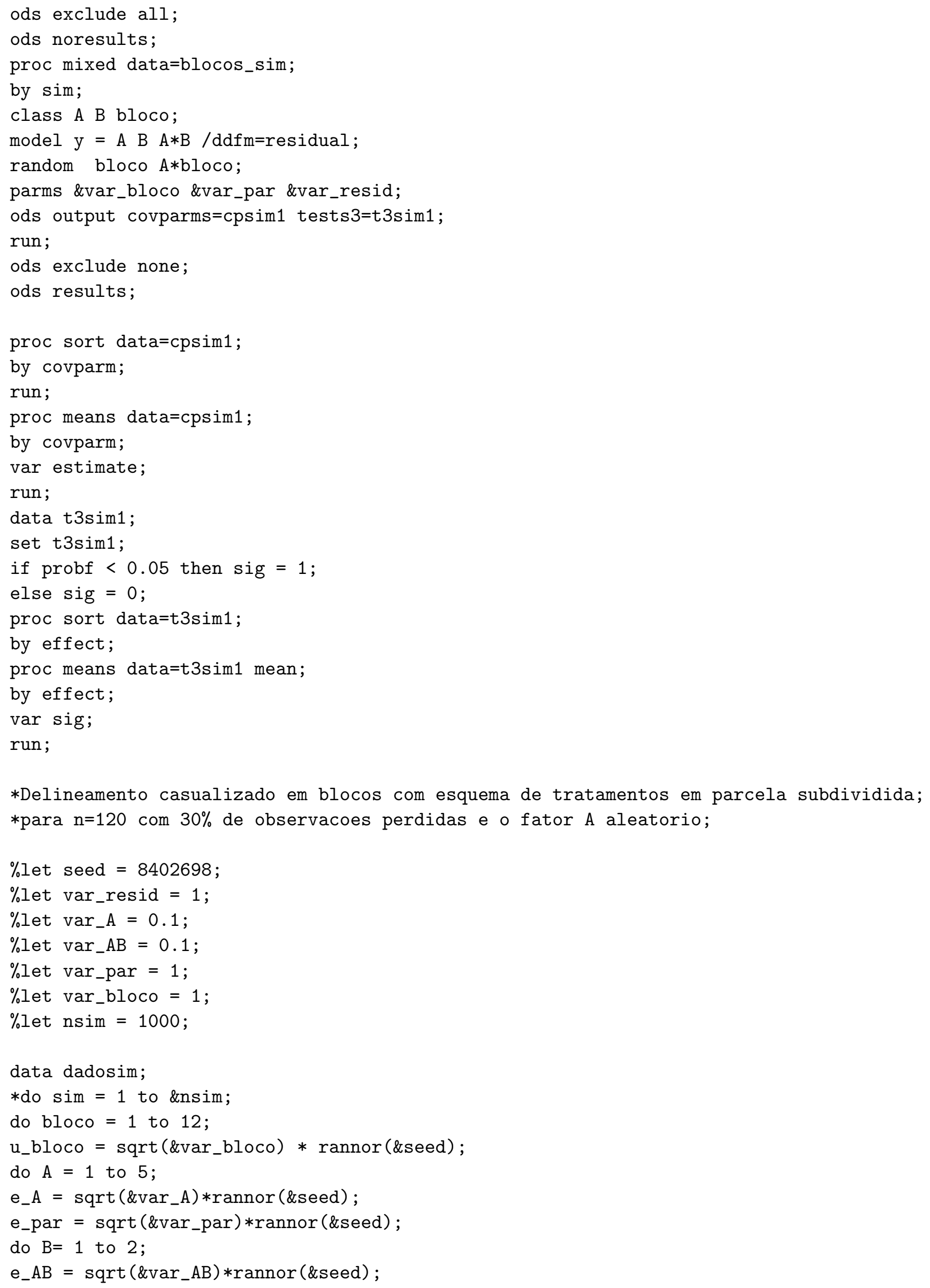


$e_{-}$sub $=$sqrt (\&var_resid) $*$ rannor (\&seed);

$\mathrm{y}=\mathrm{u}_{-}$bloco $+\mathrm{e}_{-} \mathrm{A}+\mathrm{e}_{-} \mathrm{AB}+\mathrm{e}_{-}$par $+\mathrm{e}_{-} \mathrm{sub}$;

if ( $b l o c o=1$ and $A=3$ and $B=2$ ) or $(b l o c o=4$ and $A=3$ and $B=1$ ) or

(bloco=5 and $A=5$ and $B=1$ ) or (bloco=6 and $A=1$ and $B=1$ ) or

(bloco=7 and $A=1$ and $B=1$ ) or (bloco=7 and $A=1$ and $B=2$ ) or

(bloco=10 and $A=2$ and $B=2$ ) or (bloco=11 and $A=1$ and $B=1$ ) or

(bloco=11 and $A=2$ and $B=2$ ) or $(b l o c o=11$ and $A=3$ and $B=2$ ) or

(bloco=11 and $A=5$ and $B=2$ ) or ( $b l o c o=5$ and $A=5$ and $B=2$ ) or

(bloco=1 and $A=3$ and $B=1$ ) or $(b l o c o=2$ and $A=1$ and $B=1$ ) or

(bloco=3 and $A=3$ and $B=2$ ) or $(b l o c o=4$ and $A=2$ and $B=2$ ) or

(bloco $=7$ and $A=2$ and $B=1$ ) or ( $b l o c o=7$ and $A=5$ and $B=1$ ) or

(bloco=8 and $A=2$ and $B=2$ ) or ( $b l o c o=9$ and $A=5$ and $B=1$ ) or

(bloco=10 and $A=3$ and $B=1$ ) or $(b l o c o=10$ and $A=4$ and $B=1$ ) or

(bloco=12 and $A=1$ and $B=2$ ) or ( $b l o c o=12$ and $A=2$ and $B=1$ ) or

(bloco=1 and $A=2$ and $B=1$ ) or $(b l o c o=2$ and $A=2$ and $B=2$ ) or

(bloco=3 and $A=4$ and $B=1$ ) or ( $b l o c o=5$ and $A=1$ and $B=1$ ) or

(bloco=6 and $A=1$ and $B=2$ ) or $(b l o c o=6$ and $A=3$ and $B=2$ ) or

( $b l o c o=7$ and $A=4$ and $B=2$ ) or ( $b l o c o=7$ and $A=5$ and $B=2$ ) or

(bloco=9 and $A=1$ and $B=1$ ) or ( $b l o c o=9$ and $A=4$ and $B=1$ ) or

( $b l o c o=10$ and $A=4$ and $B=2$ ) or $(b l o c o=12$ and $A=2$ and $B=2)$ then do;

$\mathrm{y}=$.;

end;

output;

end;

end;

end;

*end;

*end;

run;

PROC PRINT DATA=dadosim;

RUN ;

PROC MIXED Data=dadosim method=REML;

CLASS A B bloco;

MODEL $\mathrm{y}=\mathrm{B} /$ solution $\mathrm{ddfm}=\mathrm{kr}$;

random $\mathrm{A} A * \mathrm{~B}$ bloco $\mathrm{A} * \mathrm{bloco}$;

ods output tests $3=$ t 1 ;

LSMEANS B/diff CL;

RUN ;

* Poder analitico do teste $\mathrm{F}$;

data f_power;

set $t 1$;

Noncen $=$ NumDF $*$ FValue;

Alpha $=0.05$;

FCrit = finv (1-Alpha, NumDF, DenDF, 0);

Power $=1-\operatorname{probf}$ (FCrit, NumDF, DenDF, Noncen);

run;

proc print data $=f_{\text {_p }}$ power; 
run;

* taxa de erro tipo I;

data aleat;

do $\operatorname{sim}=1$ to \&nsim;

do bloco = 1 to 12 ;

$\mathrm{u}_{-} \mathrm{bloco}=\operatorname{sqrt}\left(\& v a r_{-}\right.$bloco $) *$ rannor (\&seed $)$;

do $A=1$ to 5 ;

$e_{-} A=\operatorname{sqrt}\left(\& v a r \_A\right) *$ rannor (\&seed);

e_par = sqrt (\&var_par) $*$ rannor (\&seed);

do $B=1$ to 2 ;

$e_{-} A B=$ sqrt (\&var_AB) *rannor (\&seed);

$\mathrm{e}_{-}$sub $=$sqrt (\&var_resid) $*$ rannor (\&seed);

aleat=u_bloco + e_A + e_AB + e-par + e_sub;

if (bloco=1 and $A=3$ and $B=2$ ) or ( $b l o c o=4$ and $A=3$ and $B=1$ ) or

(bloco=5 and $A=5$ and $B=1$ ) or (bloco=6 and $A=1$ and $B=1$ ) or

(bloco $=7$ and $A=1$ and $B=1$ ) or (bloco=7 and $A=1$ and $B=2$ ) or

(bloco=10 and $A=2$ and $B=2$ ) or (bloco=11 and $A=1$ and $B=1$ ) or

(bloco=11 and $A=2$ and $B=2$ ) or $(b l o c o=11$ and $A=3$ and $B=2$ ) or

(bloco=11 and $A=5$ and $B=2$ ) or (bloco=5 and $A=5$ and $B=2$ ) or

(bloco=1 and $A=3$ and $B=1$ ) or (bloco=2 and $A=1$ and $B=1$ ) or

(bloco $=3$ and $A=3$ and $B=2$ ) or (bloco=4 and $A=2$ and $B=2$ ) or

(bloco $=7$ and $A=2$ and $B=1$ ) or (bloco=7 and $A=5$ and $B=1$ ) or

(bloco $=8$ and $A=2$ and $B=2$ ) or (bloco=9 and $A=5$ and $B=1$ ) or

(bloco=10 and $A=3$ and $B=1$ ) or $(b l o c o=10$ and $A=4$ and $B=1$ ) or

(bloco=12 and $A=1$ and $B=2$ ) or (bloco=12 and $A=2$ and $B=1$ ) or

(bloco=1 and $A=2$ and $B=1$ ) or ( $b l o c o=2$ and $A=2$ and $B=2$ ) or

(bloco $=3$ and $A=4$ and $B=1$ ) or (bloco=5 and $A=1$ and $B=1$ ) or

(bloco $=6$ and $A=1$ and $B=2$ ) or (bloco=6 and $A=3$ and $B=2$ ) or

(bloco $=7$ and $A=4$ and $B=2$ ) or (bloco $=7$ and $A=5$ and $B=2$ ) or

(bloco=9 and $A=1$ and $B=1$ ) or (bloco=9 and $A=4$ and $B=1$ ) or

(bloco=10 and $A=4$ and $B=2$ ) or $(b l o c o=12$ and $A=2$ and $B=2)$ then do;

aleat $=$.;

end;

output;

end;

end;

end;

end;

run;

PROC PRINT DATA=aleat;

RUN;

ods exclude all;

ods noresults;

proc mixed data=aleat;

by sim;

CLASS A B bloco;

MODEL aleat $=B / \operatorname{ddfm}=k r$; 
random $\mathrm{A} A * \mathrm{~B}$ bloco $\mathrm{A} * \mathrm{bloco}$;

parms \&var_A \&var_AB \&var_bloco \&var_par \&var_resid; ods output covparms=cpsim tests3=t3sim;

run;

ods exclude none;

ods results;

proc sort data $=$ cpsim;

by covparm;

run;

proc means data $=$ cpsim;

by covparm;

var estimate;

run;

data t3sim;

set t3sim;

if probf $<0.05$ then sig $=1$;

else $\mathrm{sig}=0$;

proc sort data=t3sim;

by effect;

proc means data=t3sim mean;

by effect;

var sig;

run;

*Poder simulado;

proc mixed data=dadosim method=REML;

CLASS A B bloco;

MODEL y $=\mathrm{B} / \mathrm{ddfm}=\mathrm{kr}$ outpm=opm;

random $\mathrm{A} A * \mathrm{~B}$ bloco $\mathrm{A} * \mathrm{bloco}$;

run;

PROC PRINT DATA=opm;

RUN ;

data opm1;

set opm;

drop y stderrpred df alpha lower upper resid;

run;

PROC PRINT DATA=opm1;

RUN ;

proc sort data=aleat;

by bloco A B;

run;

PROC PRINT DATA=aleat;

RUN;

data blocos_sim;

merge aleat opm1;

by bloco A B; 


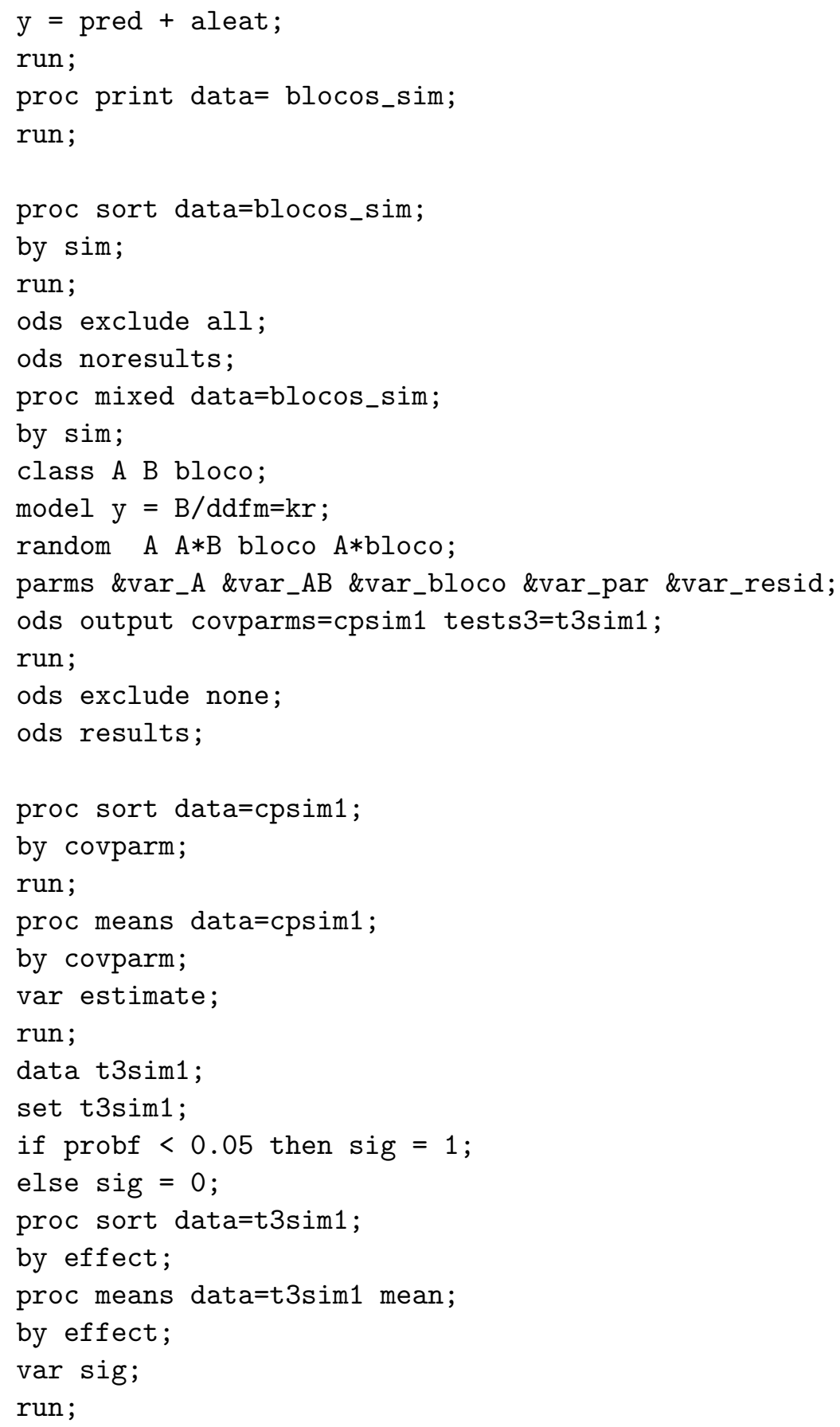

